AUSTRALIAN MUSEUM， SYDNEY. $\because x$

\author{
(CATALOGUE No. 15.)
}

$1892 x$

v. $1-3$

\title{
CATALOGUE
}

\author{
OF THE
}

\section{MARINE SHELLS OF AUSTRALIA AND TASMANIA.}

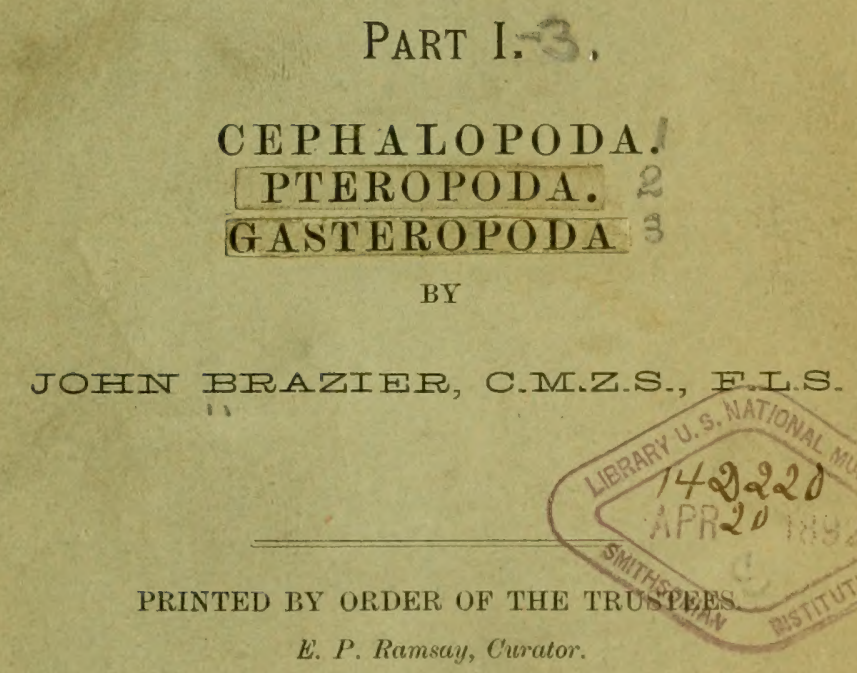

Sगोiney :

CHARLES POTTER, GOVERNMENT PRINTER, PHLLIP-STREET. 



\title{
LIST OF THE CEPHALOPODA IN THE COLLECTION OF THE AUSTRALIAN MUSEUM.
}

\author{
Bर J. BRAZIER.
}

Argonauta argo, Linné. Shells and animal in spirits.

Port Stephens, New South Wales.

Argonatta nodosa, Solander. Shells and animal in spirits.

Port Stephens, New South Wales; Tasmania.

Argonauta hians, Solander.

China Sea and South Atlantic.

Octopus gravelatus, Lamk.

Port Jackson.

Octopus australis, Hoyle.

Port Jackson.

Octopts PICTUs, Brock.

Port Jackson ; Tasmania.

Octopus Lundiates, Quoy et Gaimard.

Uji Island, Solomon Islands.

Octopus machopds, Risso.

Port Jackson, New South Wales; one foreign.

Octopus ctanea, Gray.

Port Jackson, New South Wales; Uji Island, Solomon Islands.

Eledone moschata, Lamk.

Bay of Naples, Italy.

Eledone Cirrosa, Lamk.

Bay of Naples, Italy.

Intotedthis stenodactila, Grant.

Port Jackson, New South Wales.

Rossia macrosoma, delle Chiaje.

Bay of Naples, Italy.

5e 33-91 
Sepioloidea lineolata, Quoy et Gaim.

Port Jackson, New South Wales; Nelson Bay, Port Stephens, New South Wales.

Spirula Peroni, Lam.

Port Jackson; Lord Howe Island ; Ovalu, Fiji ; New Zealand.

Sepia plangon, Gray.

Port Jackson.

Sepia apama, Gray.

Port Jackson.

SePia INdica, d'Orb.

Strait Settlement.

Sepia mestus, Gray.

Port Jackson.

Sepiotedthis lundlata, Quoy et Gaimard.

Port Jackson, New South Wales; Bougainville Island; Solomon Islands.

Sepioteuthis Australis, Quoy et Gaim.

Port Jackson, New South Wales.

Sepiotedthis Lessoniana, d'Orb.

Frazer's Island, Queensland; Uji Island, Solomon Islands; New Zealand.

Sepiotedthis Madritiana, Quoy et Gaimard.

Port Jackson, New South Wales.

Loligo Duvatceli, d'Orb.

Karache, India.

Loligo Australis, Gray.

Port Jackson.

Loligo edults, Hoyle.

Port Jackson.

Loligo Suma'trensis, d'Orb.

Malacea. 
Loligo Pealei, Lesueur.

Atlantic coast of the United States of America.

Ommastrephes gigas, d'Orb.

Lord Howe Island; Perim Island, Red Sea ; Bougainville Island,

Solomon Islands.

Todarades sagittatus, Lamk.

Bay of Naples, Italy.

Onychotedthis Bankst, Leach.

South Pacific, jumped on board H.M.S. "Comorant" Lat. 21 S., Long. 163 E., off the island of Tristan d'Achuna, presented by Captain Goddard, ship "Parramatta."

Nautilus pompilius, Linné. Shell and animal in spirits.

Woodlark Island; Torres Straits ; New Caledonia ; New Hebrides.

Nautilus stenomphalds, Sowerby.

Torres Straits.

Nautilus macromphatus, Sowerby. Shell and animal.

Isle of Pines, New Caledonia.

Nautilus umbilicatus, Lister.

Solomon Islands. 



\title{
AUSTRALIAN MUSEUM, SYDNEY.
}

(CATALOGUE No. 15.)

\section{CATALOGUE}

OF THE

\section{MARINE SHELLS OF AUSTRALIA AND TASMANIA.}

\author{
PART I. \\ CEPHA LOPODA.
}

BY

JOFIN BRAZIER, C.M.Z.S., F-I.S.

PRINTED BY ORDER OF THE TRUSTEES.

E. P. Ramsay, Curator.

Spuney :

CHARLES POTTER, GOVERNMENT PRINTER, PHILLIP-STREET. 


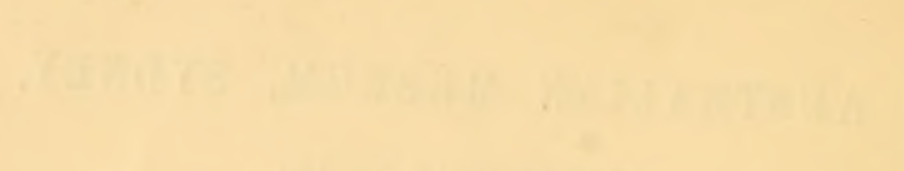

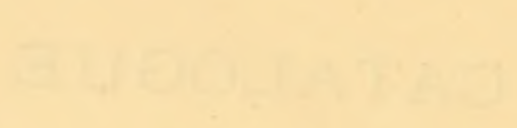

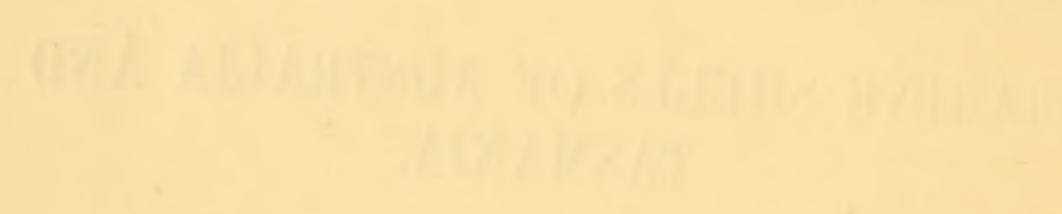




\section{PREFACF.}

Tre Trustees of the Australian Museum laving decided that a Catalogue of the Australian Mollusca should be preparel, Mr. J. Brazier, C.M.Z.S., F.LS, has been entrusted with the compilation of this work; it will necessarily be protracted, as it entails the examination of many thousands of specimens, both dry and in spirits.

The Catalogue, which will be issued from time to time in parts, will embrace not only the species represented in the general Museum Collection, but also those in the Hargrave's Collection (so liberally presented to the Trustees by the late Thomas Walker, Esq.) and those recently purchased from Mr. Brazier. The labels on the tablets of the respective specimens indicate from which collection they have been derived.

The principal synonyms and references have been added, and the letters (A.M.) after the localities indicate that the specimens are in the Australian Museum Collection.

E. P. RAMSAT, Curator. 



\section{CATAIOGUE}

oF

\section{MARINE SHELLS OF AUSTRALIA AND TASMANIA.}

\section{PART I.-CEPHALOPODA.}

IN this Part of the Catalogue are enumerated all the species of the recent Australian Cephalopoda, known to the Author or which have been described, from the coasts of Australia and Tasmania.

In compiling this portion of the Catalogue I have availed myself of the information contained in Mr. William Evans Hoyle's Report on the Cephalopoda, collected during the voyage of H.M.S. "Challenger."

JOHN BRAZIER. 



\title{
Class CEPHAL()PODA, Cuvier.
}

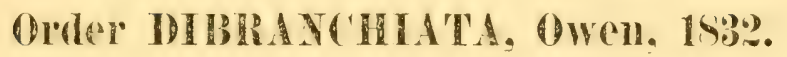

\author{
Sub-order OC'OPODA, Leach, 1818.
}

Family ARGONAUTID E, Cantraine, 1st.

A REONAL'TA, Linné, 1756.

Argonauta argo, Linné.

Argonaut" argo, Linn., Syst. Nat., ed X, p. 70S, No. 282, 231, 1758.

Nautilus papyraceus, Martini, Conch. Cab., Vol. I, p. 231, pl. 17, fig. 157, 1769.

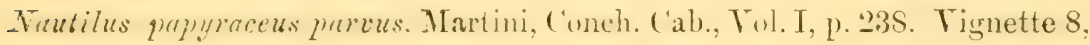
fig. 2, [. 221, 1769 .

Argonauta striata, Perry, Conch. pl. 42, fig. 2, 1811.

Argonauta grandiformis, Perry, Conch. pl. 42, fig. 4, 1811.

Argonauta Taustrum, Dillwyn, Catal., Vol. I., p. 335, 1S17.

Argonauta argo, d'Orb., C'éph. Acét. p. 15s. Argonaute, pl. 2, figs.1-2, $1835-18+8$.

Argonauba Gruneri, Dunker, Keitsehr. f. Malak, p. 18, 1852.

Argonautas argo, Reeve, Conch., Tuon., pl. 3, fig. 2c, 1861.

Argonanta hustrum, Reeve, Conch., leom., pl. 2, 1861.

Argonnuta asgo, Augas, Marine Mollusea of Port Jackson, Proc. Zool. Soc., L.ond., p. 185, 1867.

Aigonauta preifica, Dall, American Journal. Conch., Vol. I11, p. 95, 1572.

Argonauta argo, E. von Martens, Ann. Mag. Nat. Hist., 3rd series, Vol. XX,

p. 101,1867 .

Argonauta argo, Tryon, Manual of Conchologs, Vol. I, p. 138, pl. 47, figs. 11.1, 112; pl. 1. figss. 116, 117, 1879.

Aryonauta argo. Hoyle. Report on the Cephalopoda, H..M.s. "C'hallenger," Koology, Fol. XYI, pp. 1, 69, 1986.

Tinbitat.-Port Jachson Heads, Manly Beach, Port Stepliens (A.MI.), New South Wales; North Queensland, and Torres Straits. 


\section{Argonauta nodosa, Solander.}

Argonauta nodosa, Solander, Portl, Catal., 76, 212(), Vol. II, p. 113, 1.7s(i. Argonauta navicula, Solander, Portl, Catal., 12, 1055 (= forma aurita), Vol. II, p. 112, 1786.

Argonauta oryzata, Meuschen, Mus. Gevers, 252, No. 133, 1787.

Argonauta tuberculata, Shaw, Nat. Hist., Misecll, Vol. XXIIl. tab. 995. 1790.

Argonauta vitrea, Perry, Conchology, pl. 42, fig. 1, 1811.

Argonauta gondola, Dillwyn, Desc. Catal., Vol. I, p. 335, 1817 (= forma aurita).

Argonauta tuberculosa, Schumacher, Essai d un Nouv. Syst., p. 260, 1817.

Argonauta tuberculosa, Lamk., Anim. s. vert., VII, p. 632, 1822.

Argonauta tuberculata, d'Orb., Céph. Acét., p. 169, Argonaute, pl. 3, 4 (pl. 4, = forma aurita), 1835.

Argonauta tuberculosa, Reeve, Conch. Icon., pl. 1, 1861 (= formu mutica).

Argonauta oryzata, Angas, Molluscan Fauna of South Australia, Proc:

Zool. Soc., Lond., p. 156, 1865.

Argonauta oryzata, Angas, Marine Mollusea found in Port Jackson, Proe.

Zool. Soc., Lond., p. 185, 1867.

Argonauta tuberculata, E. von Martens, Ann. Mag. Nat. Hist., 3rd seriew.

Vol. XX, p. 104, 1867.

Argonauta nodosa, Tryon, Manual of Conchology, Vol. I. p. 110, pl. 50. fig. 124, 1879.

Argonauta tuberculosa, Hutton, Manual of New Zealand Mollusea, p. 2. 1880.

Argonauta tuberculata, Hoyle, Report on the Cephalopoda, H.M.S, "Challenger," Zoology, Vol. XVI, p. 4, No. 2, 1886.

Habitat.-Outer Manly Beach, Neweastle, Port Stephens (A.MI.), New South Wales; Tasmania (A.M.), South Australia, New Zealand.

\section{Argonauta hians, Solander.}

Nautilus tenuis, Martini, Conch. Cab., Tol. I, p. 235, pl. 17, figs.. 1.5, 9, 1769.

Argonauta hians, Solander, Portl, Catal., p. 4t, lot 1,055, Vol. II, p. 11:3, 1786.

Argonauta nitida, Lamk., Anim. sans Vert., t. VII, p. 653, 1822.

Argonauta gondola, Dillwyn, Vol. I, p. 335, No. 4, 1817. 
Argonauta hians, Dillwyn, Vol. I, p. 334, No. 3, 1817.

Argonauta hians, Ad. and Reeve, Voy. "Samaraug," Mollusca, part 1, p. 4, No. 2, pl. 3, fig. $2^{\mathrm{a}, \mathrm{b}, \mathrm{c}}, 1848$.

Argonauta hians, d'Orb., Céph. Acét, p. 174, Argonaute, pl. 5, 1835.

Argonauta gondola, Ad. and Reeve, Voy. "Samarang," p. 3, No. 1, pl. 2, 1848.

Argonauta Owenii, Ad. and Reere, Voy. "Samarang," p. 4, No. 3, pl. 3, flg. $1,1848$.

Argonuzta hyans, Gray, Ceph. Antep., Brit. Mus. Cat., p. 33, 1819.

Argonauta Kochiana, Dunker, Zeitschr. f. Malak, p. 49, 1852.

Argonauta gondola, Reere, Conch. Icon., pl. 4, figs. $3^{a}-3^{b}, 1861$.

digonautu hians, Hoyle, Report on the Cephalopoda, II.AI.S. "Challenger," Zoology, Vol. XVI, p. 5, No. 3, 1886.

Habitat.-Moreton Bay, Queensland. (Strange.)

\section{Argonauta Böttgeri, Maltzan.}

Argonauta Böttyeri, Maltzan, Tournal de Conchyliologie, Vol. XXIX, p. 163, pl. 6, fig. 7, 1881.

Argonaula Böttgeri, E. A. Smith, Ann. and Mag. Nat. Hist., Vol. XX, 5th series, p. 109, pl. 17, figs. 1-6, 1887.

Mabitat.-Australia.

Family OCTOPODID A, d'Orbigny. OCTOPUS, Lamarck, 1799.

\section{Octopus Boscii, Lesueur.}

Sepia rugosa, Peron, MIS.

Sepia Bosrii, Lesucur, Journal Acad. Nat. Sci., Philard, Vol. II, p. 101, 1821.

Octopus variolatus, Peron, Blainr., Diet. de Sci. Nat., t. XLIII, p. 1S6, 18.6. Octopus Boscii, d'Orb., Mon. des Céph. Acét., p. 68, 1838.

Octopus Boscii, Gray, Brit. Mus. Catal., p. 12, 1849.

Ortopus Boscii, var pallida, Hoyle, Diagnoses I, 1. 223, 1S55; II.MIS.

"Challenger," Report, Zoology, Vol. XVI, pp. 81, 82, pl. 1; pl. 2, fig. 2, 1886.

Mabitat.-Western Australia; East Moneour Island, Bass Strait, 39 fathoms, sand and shells; Twofold Bay, New South Wales, 150 fathoms, green mud. ("Challenger.") 


\section{Octopus granulatus, Lamarck.}

Polypus mas, Seba, Thes. III, pl. 2, fig. 2, 3 (?), 1758.

Octomes. Barlier, Phil. Trans, Vul. L, part', p. 777. pl. 29, fig. 1. 4, original figures from Edwards, 1755.

Octopus. Bruguire. Encycl. Meth.. pl. 76, fig. 1, 2, 17S3; figures copied from Sebar.

Sepia o'ugosa, Bosc., Actes de la Soc. d'Hist. Nat., tab. 5, fig. 1-2, 1792.

Ortopus yionulutus. Tamarek. Mím. de la Soc. d'Hist. Nat. de Paris, t. I, p. 20, No. 2, 1799.

Sepia granulosa, Bosc., Buff. de Déterville Vers., t. I, p. 47, 1802.

Le Poulpe granuleux, Montfort, Buff. de Somnini, MLll., t. III, p. 30. pl. 29), 1802 ; very bad copy ; Bosc. figure.

Le Poulpe Amerienin, de Barker. Montfort, Buff. de Sommini, MIoll., t. III. p. 3S, pl. 30 (figure copied from Barker, fig. 3) ; pl. 31, fig. 1 (copied from Barker, fig. 2, and copied from Seba, fig. 2.)

Octopus granulatus, Lam., Anim. s. Vert., t. VII, p. 65S, 1822.

().topus yromulatus, Fer. dOrb., Tableaus des Céphal, p. 53, o. 2, 1826.

Octopus gramulatus, Blainrille, Dict. des Sci. Nat., XLIII. p. 1S5, 1S26.

Octopus Bavkeri, Fer. d’Orb., Tab. des Céph., p. 54, No. 3, 1826.

Octopus rugosus, Blainville, Dict. des Sei. Nat., XLIII, p. 185, 1826.

Octopus anticanus, Blainville, Diet. des Sei. Tat., XLIII, p. 199, 1862.

Uctopus ingosus, l'Orbiguy et Ferussac, Mem. des Cíphal. Acét., p. 45. No. 9, Poulpes, pl. 6, figs. 1, 2. O.granulatus, pl. 23, fig. 20. O. rugosus, very poor figure of Bosc.

Octopus polyzenia, Gray, Ceph. Antep., Brit. Mus., p. 13, 1849.

Orfopus incertus. Tarc., Cet. Mus. Firenze, p. 22, tar. VI, figs. 9, 11, 1569.

Octopus polyzenia, E. A. Smith, "Alert" Report, p. 34, pl. 4, figs. A, A3, 1854.

Octopus qranulatus, Iorle, Report on the Cephalopoda, H.MI.s. "Challenger," Zoology, Tol. XYI, pp. 8, S0, No. 6, 1856.

Octomus polyzeniu. Horle, Report on the Cephalopoda, H.M.S. "Challenger," Zoology, Tol. XII, p. S, No. 9, 1856.

Habitat. -- Port Essington. North Australia, Thursday Island, Torres Straits, 4 to 5 fathoms; Port Jackson (1.MI.), Port Kembla, Broken Bay, Port Stephens, Newcastle, and the New South Wales coast in general. Moreton Bay, Queensland. 
Octopus tetricus, Gould.

Octopus tetricus, Gould., Moll. Wilkes Exped., p, 174, fig. 558, 1552.

Octopus tetricus, Tryon, Manuil of Conehologg, lul. 1. p. 1:21, pl. 3.5. Ligr. Hi. 47.

Octopus tetricus, Hoyle, H.M.S. "Challenger," Report, 18s6. Tol. IVI, pp. 8, 80, 82.

Habitut.-Found near Sydney, Now South Walex. (Willies Experlition.)

Octopus superciliosus, Quoy ct Gaimard.

Octopus superciliosus, Quoy et Gaimard, Voy. "Astrolabe," t. II, p. 88, pl. 6, fig. 4, 1832.

Octopus Westernicnsis, Quog. d'()rb., Céph. Acit., l'oulpes. pl. I, fig. :3, 1×:3৬. Octopus superciliosus, d'Orb., Céph. Acét, p. 41, pl. XXVII, fig. 9.

Octopus supercitiosus, Gray, Cat. Ceph. Antep., p. 12, ํo. 1S, 1849.

Octopus supercitiosus, Hoyle, Report on the Cephalopodil. H.ML.s. "Challenger," Vol. XVI, p. 9, No. 16, 1856.

Habitat.-Western Port, Bass Strait, Victoria.

\section{Octopus Australis, Hoyle.}

Octopus australis, Hoyle, Diagnosis I, p. 224, 185.5.

Octopus australis, Hoyle, Prelim. Rep. I, p. 9s, 1885.

Octopus australis, Hoyle, Report on the Cephalopoda, II.ML.'. " Challenger," Zoology, Vol. XVI, pp. 9, s8, pl. 3, figs. t, 5, 1886.

Habitat.-George's Beach, and 6 to 15 fathoms, Port Jackson, New South Wales. (A.M.)

Octopus tenebricus, E. A. Smith.

Octopus tencbricus, E. A. Smith, "Alert" Report, p. 35, p1. 4, fig. B, 1854. Octopus tenebriezs, Hoyle, Report on the Cophalopodar. II.M.s. " ('hallenger." Zoology, Vol. XVI, p. 9, No. 22, 18s6.

Habitat.-Port Denison, Queensland, 3 to t fathoms. (H.MI.S. "Alert.")

Octopus duplex, Hoyle.

Octopus duplex, Hoyle, Diagnosis I, p. 226, 1885.

Octopus duplex, Hoyle, Prelim. Report, I, p. 101, 1585.

Octopus duplex, Inoyle, Report on the ('ephalupoda, M.M.s. " ('hallentrer." Zoolooy, Vol. XVI, pp. 10, 90, pl. 7, fig. 5, 1886.

Habitat.-Off Twofold Bay, New South Wales, 150 fathoms. (II.M.S. "Challenger.") 
Octopus pictus, Brock.

Octopus pictus, Brock., Keitschr, f. wiss. Zool., Bd. XXXVI, p. 603, Tilf. XXXVII, fig. 3, 1882-(non Octopus pictus, Verrill),

Octopus maculosus. Hoyle, Proc. Roy. Phys. Soc. Eilin., Vol. VII, p. 319, pl. 6, 1883 .

Octopus maculosus, E. A. Smith, "Alert" Report, p. 36, pl. 4, fig. C., 1884.

Octopus pictus, Brock., var. fusciutu, Hoyle, Report on the Cephalopoda, H.M.S. "Challenger," Zoology, Vol. X V I, pp. 10, 94, 1886.

Habitat.-Port Jackson, Port Stephens, New South Wales; Tasmania (A.M.) ; Kangaroo Island, South Australia: Moreton Bay, Queensland.

\section{Octopus lunulatus, Quoy et Gaimard.}

Octopus Tunulatus, Quoy et G., Voy. "Astrolabe," t 1I, p. 86, pl. 6, figs. 1, 2, 1832.

Octopus Tunulatus, d'Orb., Céph. Acét., p. 59, Poulpes, pl. 10, fig. 2, pl. 26 , figs. $5,7,1838$.

Octopus lunulatus, Gray, Ceph. Antep., Brit. Mus., p. 11, 1849.

Octopus Tunulatus, Tyron, Manual of Conchology, Vol. I, p. 121, pl. 26, figs. $15,16,1879$.

Octopus Iumulatus, IJoyle, Reporton the Cephalopodi, II.M.S. "Challenger," Zoology, Vol. XVI, p. 10, No. 36, 1S86.

Habitat.-Torres Straits; Uji, Solomon Islands. (A.M.)

\section{Octopus macropus, Risso.}

Octopus macropus, Risso, Hist. Nat. Eur. Mérid., t. IV, p. 3, 1826.

Octopus Lechenaultii, d'Orb., 'Tab. des Poulpes, pl. I, 1825.

Octopus Cuvierii, d'Orb., Céph. Acét., Poulpes, pl. 4, 1826.

Octopus macropodus, Sangiovani, Ann. Se. Nat., t. XVT, p. 319, 1829.

Octopus longimanus, Féruss, Poulpes, pl. 2, 1824.

Octopus Cuvierii, d’Orb., Céph. Acét., p. 18, Poulpes, pl. 4, 24, 1835.

Octopus macropus, Risso, d’Orb., Céph., Poulpes, pl. 24, 1835.

Octopus Cuvierii, Gray, Céph. Ante. Brit. Mus. Cat., p. 13, 1849.

Octopus macropus, Verany, Céph. Médit., p. 27, pl. X, 1851.

Octopus macropus, I’arg., Céph. Mus. Firenze, p. 23, 1869.

Octopus Cuvicrii, Targ., Ceph. Mus. Firenze, p. 24, 1869. 
Octopus Cuvierii, Appellöf, Japanska Cep., p. 6, pl. 1, fig. 6, 1886.

Octopus macropus, Hoyle, Report on the Cephalopoda, H.MI.S. "Challenger," Zoology, Vol. XVI, pp. 11, 95, 1886.

Habitat.-Port Jackson, New South Wales. (A.M.)

\section{Octopus cyanea, Gray.}

Octopus cyanea, Gray, Ceph. Antep., Brit. MLus. Cat., p. 15, $18 \pm 9$.

Octopus cyanea, Tryon, Manual of Conchology, Vol. I, p. 124, 1879.

Octopus cyanea, IIoyle, Report on the Cephalopodia, II.ML.s. "Challenger," Zoology, Vol. XVI, p. 12, No. 58, 1886.

Habitat.-Port Jackson, New South Wales (A.M.); Moreton Bay, Queensland; Uji Island, Solomon Islands.

\section{Octopus Peronii, Lesneur.}

Sepia Octopoda, Peron, MSS.

Sepia Peronii, Lesueur, Journ. Acad. Nat. S'c.. Philad., Vol. II, p. 101, 1821. Octopus Peronii, Férus, d’Orb.. Tab. Class. des Céph., 54, No. 7, 1823.

Octopus pustulosus, Peron, Blainv., Diet. Sc. Nat., XLIII, p. 186, 1826.

Octopus pustulosus, d'Orb., Céph. A cét., p. 66, 1835, 1848.

Octopus Peronii, Hoyle, Report on the Cephalopoda, H.M.S. "Challenger," Zoology, Vol. XVI, p. 13, No. 68, 1886.

Habitat.-Australian Seas. (Peron.)

\section{Octopus cærulescens, Peron.}

Octopus carulescens, Peron, Blainv., Dic. des Sc. Nat, t. XLII, p. 129, 1826. Octopus carulescens, d'Orb., Céph. Acét., p. 65, 1835-48.

Octopus carulescens, Hoyle, Report on the Cephalopoda, II.M.S. "Challenger," Zoology, Vol. XVI, p. 13, No. 73, 1886.

Habitat.-Australian Seas. (Peron.)

\section{Octopus cornutus, Owen.}

Tritaxeopus cormutus, Orren, Trans. Zool. Soc. London, Vol. XI, part. 5, p. 131, pl. XXIII, 1881.

Octopus cornutus, Hoyle, Report on the Cephalopoda, H.M.S. "Challenger," Zoology, Vol. XVI, p. 14, No. 78, 1886.

Habitat.-Australian Seas. 


\section{Octopus sp.}

Octopus sp. Hoyle, Report on the Cephalopoda, II.M.S. "Challenger," Zoology, Vol. XVI, p. 101, 1886.

Habitat.-Station 18s, south of Papua, lat. $9^{\circ} 59^{\prime}$ S., long. $139^{\circ} 42^{\prime}$ E. ; 29 fathoms; green mud. (H.M.S. "Challenger.")

\section{Octopus sp.}

Octopus sp. Hoyle, Report on the Cephalopoda, H.M.S. "Challenger," Zoology, Vol. XVI, p. 101, 1886.

Habitat.-Station 184, east of Cape Tork, lat. $12^{\circ} 8^{\prime}$ S., long. $145^{\circ} 10^{\prime}$ E.; 1,400 fathoms; Globigerina ooze. (iI.M.S. "Challenger.")

\section{Sub-order DECAPODA, Leach. \\ Drrision MYOPSIDA, d'Orbigny. \\ Family SEPIOLINI, Steenstrup. \\ SEPIOLA (Rondelet, 1554); Leach, 181\%.}

\section{Sepiola Rondeletii, Leach.}

De Sepiola, Rondelet, De Piscibus, lib. XVII, cap. X, p. 519, 1554.

Sepiola Rondeletii, Gesncr, De Aquat., lib. IV, p. 855, 1558.

De Sepiola, Boussuet, De Nat. Aquat., p. 201, 1558.

Sepiola Rondeletii, Aldrovande, De Mollib., lib. T, p. 63, 1642.

Sepiola, Jonston, Hist. Nat. De Piscibus, lib. I, cap. III, t. I, p. S, fig. S, 1650 .

Sepiola, Ruysch, Theatr. Erang., t. 1, fig. 1, 1718.

Sepia sepiola, Linn., Syst. Nat. edit. 12, p. 1,096, No. 5, 1767.

Sepia sepiola, Scopoli, Observ. Zool., p. 12S, 1772.

Sepia sepiola, Herbst., Winiseit. zur Kennt. der Gerr., p. 80, No. 4, 1785.

Sepia sepiola, Gmelin, Syst. Nat., ed. 13, p. 3,151, No. 5, 1789.

Loligo sepiole, Lamarek, Mémoires de la Société d'Ilist. Nat. de Paris. p. 16, 1799.

Sepiola Rondeletii, Lench, Zool. Miscell., Vol. III, p. 13\$, 1817.

Loligo sepiola, Lamarck, Anim. sans. Vert., t. VII, p. 664, No. 4, 1822.

Sepiola vulgaris, Grant, Trans. Zool. Soc., Lond., Vol. I, p. 77, 1833.

Seppiala Rondeleti, d’orh., (“ph. Acút., p. 2:30; Śppioles, pl. 1, figs. 1, 6: pl. 2, figs. 3,13 ; pl. 3, figs. $6,9,1835-4$. 
Sepiola Sepiola, Tryon, Manual of Conchology, Tol. 1, p. 155. pi. (66, fieg. 229, 1879.

Sepiola Rondeleti, IIoyle, Report on the Cephalopodi, II.M.'. "Challenger," Zoology, Vol. XVI, p. 101, 1856.

Habitat.--Iron Stone Cove, Port Jackson, N.S. W. (J. Brazier.)

INTOTEUTHIS, Verril1, 1 ssi.

Inioteuthis stenodactyla, Grant.

Sepiola stenodactyla, Grant, Trans. Zool. Soc., I figs. 1, 2, 1833.

Sepiola stenodactyla, Gervais et Van Beneilen. Bull, de l'Acad. de Bruxelles, t. V, No. 7, from Grant.

Sepiola stenodactyla, d’Orb., Céph. Acét., p. 235, X̄o. 5 ; Sépioles, pl. 2, figs. $1,2,6$ c, $1835-48$.

Sepiola stenodactyla, Gray, Ceph. Antep., Brit. Mus. Cat., p. 94, 1s49.

Sepiola stenodactyla, Tryon, Manual Conchology, Vol. I, p. 157, pl. 66., fig. 239,1879 .

Sepiola stenodactyla, Hoyle, Report on the Cephatopoda, II.MI.... " ("hailenger," Zoology, Vol. XVI, p. 17, No. 5, 1886.

Habitat.-Port Jackson, New South Wales. (A.Mr.)

\section{Inioteuthis Tasmanica, Pfeffer.}

Sepiola tamanica, Pfeffer, Ceph. Hamb. Mus., p. 6, fig. 7, 1854.

Sepiota tasmanica, Inoyle, Report on the Cephalopodil, H.M.... " C'hallemger," Vol. XVI, p. 17, 18s6.

Habitat.-Tasmania and Bass' Strait.

Family, SEPIARII, Steenstrup, 1861.

SEPID E, d'Orb. (Pars).

Sub-family, SEPIADARII, Steenstrup, 1851. SEPIOLOIDEA, d'Orbigny, 1539.

Sepioloidea lineolata, Quoy et Gaimard.

Sepiola lineolala, Quoy et Gaim., Zool., Voy. "Astrolabe," Vol. II, p. S2, Moll., pl. 5, figs. S, 13, 1832.

Sepiola lineolata, Gervais et Van Beneden, t. T, p. 6, No. $7,1838$. 
Sepioloidea lineolata, d’Orb., Céph. Acét., p. 210 ; Sépioles, pl. 3, figs. 10, 18, 1839.

Sepiola lineata, Gray, Ceph. Antep. Brit. Mus., p. 95, 1849.

Sepiola lineolata, Tryon, Manual Conchology, Vol. I, p. 157, pl. 66, fig. 242 ;

pl. 67, figs. 240, 241, 243, 1879.

Sepioloidea lineata, Stp., Sepiadarium og Idiosepius, p. 233, 1881.

Sepioloidea lineata, Brock, Zeitschi, f. Wiss. Zool., Bd. IL, pp. 105, 120, 18 st.

Sepioloidea lineolata, Hoyle, Report on the Cephalopoda, H.M.S. "Challenger," Zoology, Vol. XVI, p. 19, No. 1, 1886.

Habitat.-Jervis Bay, Port Jacksnn, New South Wales (A.M.), Port Stephens; St. Vincent Gulf, South Australia.

\section{Sub-family, IDIOSEPII, Steenstrup, $18 \$ 1$.}

SPIRULA, Lamarck, 1801.

\section{Spirula Peroni, Lamarck.}

Nautilus spirula, Linné, Syst. Nat., 12th edition, p. 1163, 1766.

Spirula fragilis, Lam., Syst. des A nim. sans Vert., p. 102, 1801.

Spirula prototypus, Peron, Voy. Terres Austral, pl. 30, fig. 4, 1804.

Spirula Australis, Lam., Ency. Meth., pl. 465, fig. 5, 1816.

Spirula fragilis, Schumacher, Nouv. Syst., p. 256, 1817.

Spirula peronii, Lam., Anim. sans Vert., Vol. 7, p. 601, 1822.

Spirula peronii, Lam., Anim. sans Vert, Desh. Edit., Vol. II, p. 280, 1815.

Spirula peronii, Owen in Ad. and Reeve, Voy. "Samarang," Moll. pl. 4, figs. $1,4,5,6,7,11,12,13,14,15,1884$.

Lituus prototypus, Gray, Ceph. Antep. Brit. Mus., p. 115, 1849.

Lituus lavis, Gray, Ceph. Antep., Brit. Mus., p. 116, 1849.

Ammonia lavis, Angas, Proc. Zool. Soc., Lond., p. 157, 1865.

Ammonia (Lituus) lavis, Angas., Proc. Zool. Soc., Lond., p. 186, 1867.

Spirula peronii, Tryon, MIanual of Conchology, Vol. I, p. 205, pl. 96, figs. $467,469,1879$.

Spimta peronii, Hoyle, Report on the Cephalopoda, Fi.M.S. " ('hallenger," Zoology, Vol. XVI., pp. 20, 122, 1886.

Habitat.-New South Wales (A.M.), South Australia, Tasmania, Queensland, Torres Straits Islands, West and North-west Australia. 
Sub-family EUSEPII, Steenstrup, 1891.

SEPIA, Linné, 1766.

Sepia plangon, Gray.

Sepia plangon, Gray, Ceph. Antep. Brit. Mus., p. 104, 1849.

Sepia plangon, Tryon, Manual of Conchology, Vol. 1, p. 194, 1579.

Sepia plangon, Hoyle, Report on the Cephalopoda, II.MI.t. "Challenger," Zoology, Tol. XVI, p. 21, 1886.

Habitat.-Port Jackson, and the coast of New South Wales. (A.M.)

Sepia Smithi, Hoyle.

Sepia Smithi, Moyle, Anu. and IIag. Nat. His., ser. 5, Vol. XVI, p. 190, 1 SS5.

Sepia Smithi, Ioyle, Report un the Cephalopoda, II.MI.S. "Challenger,"

Zoology, Tol. XVT, pp. 21, 124, pl. 15, figs. 1, 12, 1856.

Habitat.-Arafura Sea, south of New Guinea; 28 fathoms; green mud. (H.MI.S. "Challenger.")

Sepia papuensis, Hoyle.

Sepria pronensis, Hoyle, Ann. and Mag. Nat. Hist., ser. 5, Vol. IVI, p. 197, 1885.

Sepra papuensis, Hoyle, Report on the Cephalopoda, H.M.S. " Challenger," Zoologg, Vol. XVI, pp. 21, 126, pl. 15, figs. 13, 23, 1886.

Habitat.--Arafura Sea, South of New Guinea; 28 iathoms; green mud. (H.M.S. "Challenger.")

Sepia latimanus, Quoy et Gaimard.

Sepria latimanus, Quoy et Gaimard, Toyage "Astrolabe," Tol. 2, p. 69, pl. 1, Mollusea, figs. 2-11, 1832.

Sepia latimanus, d'Orb., Ciph. Acít.. n. 2sis; Seiches, pl. 12, figs. 1, 6, pl. 1s, figs. 16, 17, 1839.

Sepia latimanus, Tryon, Manual of Conchology, Vol. I, p. 192, pl. SS, figs. 400, 401, 1879 .

Sepia latimanus, Gray, Ceph. Antep., Brit. Mus., p. 101, 184.

Habitat.-Western and North-west Australia; Torres Straits.

Sepia apama, Gray.

Sepia apama, Gray, Ceph. Anten. Brit. Mus, p. 103, No. 10, 1849.

Sepia apama, Tryon, Manual of Conchology, Vol. I, p. 194, 1879.

Sepia apama, Hoyle, Report on the ('ephalepoda, IL.MI.s. "Challenger," Zoology, Vol. XVI, p. 24, 1886.

Habitat.-Port Jackson, and the coast of New South Wales; South Australia. (A.M.) 
Sepia indica, d'Orbiguy.

Sepia Blainvillei, d'Orb., Céph. Acét., pl. 21, 1839 (not Deshayes, 183i).

Sepia indica, d'Orb., Céph. Acét., p. 2ss, No. 21, 1839.

Sepia indica, Gray, Ceph. Antep., Brit. Mus., p. 108, 1819.

Sepia indica, Hoyle, Report on the Cephalopoda, H.MI.S. "Challenger," Zoology, Vol. XVI, pp. 22, 21s, 220.

Habitat.-Testern Australia, Cape Upstart, N.T. Australia.

\section{Sepia rostrata, d'Orbigny.}

Sepia rostrala, d’Orb., Céph. Acét., p. 281; Seiches, pl. 8, fig. 6, pl. 26, 1839. Sepia rostrala, Gray, Ceph. Antep., Brit. MLus., p. 10S, 1819.

Acanthosepion spinigerum, Rochebr., Monogr. Sepiadse, p. 103, 1 S81.

Sepia rostrata, Hoyle, Report on the Cephalopoda, II.M.S. "Clrallenger," Zoology, Vol. XVI, pp. 22, 2LS, 220.

Habitat.-Western Australia and Torres Straits.

\section{Sepia Australis, d'Orhigny.}

Sepia Australis, d'Orb., Céph. Acét., p. 285̃ ; Seiches, pl. 7, fig. 4, 1839.

Sepia Australis, Inoyle, Report on the Cophalupont., II.AI.S. " ('hallenger," Zoology, Vol. XVI, pp. 22, 220.

Hubitat.--Kangaroo Island, South Australia.

\section{Sepia mestus, Gray.}

Sepia mestus, Gray, Ceph. Antep. Brit. Mus., p. 10S, 1819.

Sepia mestus, Hoyle, Report on the Cephalopoda, II.AL.S. "Challenger," Zoólogy, Vol. XVI, p. 23, 220, 1856.

Hatitat, Port Jackson, and the Coast of New South Wales. (A.MI.)

\section{Sepia cultrata, Hoyle.}

Sepia cultrata, Stp., M.S. in Mus., Harn.

Sepia cultrata, Hoyle, Ann. and Mag. Nat. Hist, series 5, Vol, XTI, p. 19S, 1855.

Sepia eultrata, Hoyle, Report on the Cephalopoda, H.M.S. "Challenger," Zoology, Tol. XVI, pp. 133, 220, pl. 20, 1587.

Sepia capensis, Var. Gray, Ceph. Antep., Brit. Mus., p. 110 (excl. syn.)

Habitat.-Twofold Bay, New South Wales; 150 fathoms; green mud. (H.M.S. "Challenger.") 
Sepia elliptica, Ifoyle.

Sepia elliptiea, Hoyle, Ann. and Mrag. Nat. Hist., serics 5, Tol. XVI, p. 1S9, 1885.

Sepia elliptica, Hoyle, Report on the Cephalopoda, II.MIS. "Challenger," Zoology, Vol. IVI, pp. 22, 131, 4S ; pl. 19, figs. 1.1, 21.

Habitat.-Arafura Sea, South of New Guinea; 25 and 49 fathoms; green mud. (IL.MI.S. "Challenger.")

Sepia capensis, d'Orbigny.

Sepia capensis, d'Orb., Céph. Acét. Seiches, pl. 7, figs. 1-3, 1826.

Sepia Australis, Quoy et Gaimard, Vor. "Astrolabe," Vol. II, p. 7, p1. 5, figs. 3. 7,1832,

Sepia capensis, d'Orb., Céph. Acét., p. 27 7 ; S'eiches, pl. 7, figs. 1, 3, pl. 12, figs. $7,11, \mathrm{pl} .17$, figs. $18,19,1839$.

Sepia Sinope, Gray, Ceph. Antep., Brit. Mus., p. 10, 181 .

Sepia capensis, Hoyle, Report on the Cephalopoda, H.MI.S. "Challenger," Zoology, Vol. XVI, pp. 23, 217.

Habitat.-Wew South Wales coast and North Queensland. (J. Brazier.)

Sepia palmata, Owen.

Sepia palmata, Owen, Trans. Zool. Soc., London, Vol. IT, part vi, page 131, pls. 22-25, 1881.

Sepia palmatr, Hoyle, Report on the Cephalopoda, H.M.S. "Challenger," Zoologs, Vol. XVI, pp. 24, 220, 1856.

Irabital.-North Queensland Coast and Norfolk Island.

Sub-Genus META SEPIA, Hoyle, ISSร̃.

Sepia (Metasepia) Pfefferi, Hoyle.

Sepia (ILelasepia) Pfefferi, Hoyle, Ann. and Mag. Nat. Hist, 5th series, Vol. XVI, pp. 199, 200, 1855.

Sepiu. (Metasepia) Pfefferi, Horle, Report on Cephalopoda, H.ML.S. "Challenger," Zoology, Vol. XVI, pp. "24, 145, 218, pl. 21, figs. 1, 10.

Habitut.-Arafura Sea, Sonth of Neir (ininea; $2 S$ fathoms; green mud. (II.MI.S. "Challenger.")

Tamily LOLIGLNEI, Steenstrup, 1861. SEPIOTEUTHIS, Blainville, 1825.

Sepioteuthis lunulata, Quoy ct Gaimard.

Sepioteuthis Tunulata, Quoy et Gaim., Toy."Astrulabe," Vol. II, p. 74, Moll., pl. 33, figs. 3, 13, 1832. 
Sepioteuthis Guinensis, Quoy et Gaim., Voy. "Astrolabe," Tol. II, p. 72, pl. 3, figs. 1, 7, 1832 .

Sepioteuthis lunulata, d'Orb., Céph. Acét, p. 300. Sépioteuthis. pl. 3, firg. 1. pl. 6 , figs. $1,8,1839$.

Sepioteuthis dorensis, d'Orb., Céph. Acét. Sépioteuthis, pl. 3, fig. 3. 1833.

Sepioteuthis Tunulata, Gray, Ceph. Antep., Brit. Mus., p. 78, 1849.

Sepiotenthis Guinensis, Tryon, Minual of Concholog., Tol. 1, p. 151. pl, 61. fig. 198, 1879.

Sepioteuthis Tunulata, Hoyle. Report on Cephalopoda, H.MI.S. " Challenger," Zoology, Vol. XVI, pp. 27, 220.

Habitat.-Port Jackson, New South Wales; Torres Straits; New Guinea; Bougainville Island; Solomon Islands. (A.M.)

Sepioteuthis Australis, Quoy et Gaimard.

Sepioteuthis Australis, Quoy et Gaim., Voy. "Astrolabe," Vol. 1I, p. 77, pl. 4, fig. 1, 1832.

Sepiothuthis Australis, d'Orb., C'́ph. Acét., p. 300; Sépioteuthis, pl. 5, fig. 5, pl. 6, figs. 15, 21, 1839 .

Sepiothuthis Australis, Gray, Ceph. Antep., Brit. Mus., p. 79, 1819.

Sepiothuthis Australis, McCoy, Nit. Hist. Tictoria, Decade 7, pl. 61.62. 1883.

Sepiothuthis Australis, Tryon, Manual of Conchology. Tol. T, p. 151. pl. (61. fig. 201, 1879.

Sepioteuthis austratis, Hoyle, Report on Cephalopoda, II.II.S. "Challenger," Zoology, Vol. XVI, pp. 26, 220.

Habitat.-Western Port, Victoria; Port Jackson, New South Wales (A.M.) ; Darnley Island, Torres Straits.

\section{Sepioteuthis Lessoniana, Férussac.}

Sepioteuthis Lessoniana, d'Orb., Tab. M[éth. Ann. de. Aci. Nat, Tol. VII, p. 155, 1826.

Sepioteuthis Lessoniana, Lesson, Toy, "Coquille," Moll., p. 211, pl. 11, 1S30. Sepioteuthis Lessoniana, d'Orb., Céph. Acét. 1). 302, Sípiotenthis, pls. 1, 6, figs. 9, 14, 1839.

Sepioteuthis Lessoniana, Gray, Ceph. Antep., Brit. Mus., p. S0, 1819.

Sepioteuthis Lessoniana, Tryon, Manual of Concholugy, Tol. T, p. 152, pl. 62, fig. 212, 1879. 
Sepioteuthis lessoniana, Hutton, New Zealand, Mollusca, p. 3, 1890.

Sepioteuthis lessoniana, Appellöf, Japanska, Ceph., p. 31, 1886.

Sepiotenthis lessoniana, IIoyle, Report on C'pl.alopoda, II.AI.S. "( "hallenger,"

Zoology, Vol. XTI, pp. 27, 219, 220.

Habitat.-Frazer's Island, Queensland; New Zealand; Uji Island, Solomon Islands (A.M.); Torres Straits; New Guinea.

Sepioteuthis mauritiana, Quoy et Gaimard.

Sopiotenthis man ritiana, Quoy et Gaim., Toy. "Astrolabe," Tol. II, p. 76, pl. 1. figs. 2, 6, 1832 .

Sepiotenthis mauritiana, d'Orb., Céph. Acét., p. 304; Sépioteuthis, pl. 5 , figs. 1, 4; pl. 7, figs. 1-5, 1839.

Sepioteuthis mauritiana, Gray, Ceph. Antep., p. 79, 1849.

Sepioteuthis manitiana, Tryon, Manual of Conchology, Tol. I, p. 1.52, 11. (62. fig. 206, 1879.

Sepioteuthis mauritiana, Ioyle, Report on Cephalopoda, II.MI.s. "Challenger," Zoology, Vol. XVI, pp. 27, 217.

Habitat.-Port Jackson, New South Wales. J. Brazier (A.M.)

Sepioteuthis bilineata, Quoy et Gaimard.

Sepru bitineata, Quay et Gaim., Voyage. "Astrolabe," Tol. II, p. 66, pl. "2. fiu. 1, 1832.

Sepioteuthis bilineuta, d’Orb., Cúph. Acét., p. 301 ; Sépiotcuthis, pl. 1, fir. .”, 1839.

Sepioteuthis bitineata, Gray, Ceph. Antep., Brit. Mus., p. 82, 1819.

Sepioteuthis bitineatu, Tryon, Manual of ('onchology, Tol. I, p. 1.5!, pl. (i:), fig. $221,1879$.

Sepioteuthis biineata, Moyle, Report on Cephalopoda, II.MI.S. "Challenger." Zoology, Vol. XVI, p. 27.

Habitat.-Western Port, Victoria; Bass Straits.

LOLIGO, Lamarck, 1799.

Loligo australis, Gray.

Loligo australis, Gray, Ceph., Antep., Brit. Mus., p. 71, 1849.

Lotigo austratis, Tryon, Manual of Conchology, Vol. I, p. 148, 1879.

Lotigo austratis, IFoyle, Report on cephalopoda, H.MI.'. " Challenerere;

Zoology, Vol. XVI, pp. 31, 220.

Mabitat.-Port Jacken, Jeweastle, Port Stephens, New South Wales (1. II.) 
Loligo edulis, Hoyle.

Lotigo edulis, Hoyle, Ann. and Mag. Nat. Hist., 5th series, Tol. TYT, p. $186,1585$.

Lotigo edulis, Hoyle, Report on Cephalopoda, H.ML.S. "Challenger," ZoologF, Vol. ITI, pp. 29, 152, pl. 23, 1886.

Habitat.-Port Jackson, New South Wales; Yokohama, Japan.

\section{Loligo indica, Pfeffer.}

Loligo indica, Pfeffer, Cephalopoda, Hamb. Mus., p. 4, fig. 3, 3a, 1854.

Loligo indica, Hoyle, Report on Cephalopoda, H.M.S. "Challenger," Zoology, Vol. XVI, pp. 29, 156, pl. 26, 1886.

Habitat.-Arafura Sea, south of New Guinea; 28, 49 fathoms ; green mud. (H.MI.S. "Challenger.")

\section{Division GGOPSID 1, d'Orbigny, 1839.}

Fanily OMMaSTREPIINI, Stenstrup, 1 s61.

Sub-family OMMASTREPHIDE, Gill, 1871.

OMITASTREPHES, d'Orbigny, 1835.

Ommastrephes gigas, d Orbigny.

Pernetti, Hist. Voy. aux Malouines, Vol. II, p. 76 ? 1770.

Scpia tunicata, Molina, Hist. Nat. du Chili, p. 173? 1789.

Sepia tunicata, Gmelin, Syst. Nat., ed. 13, p. 3151, sp. 8? 17 S9.

Sepia. nigra, Bose, Hist. Nat. des Vers, Vol. I, p. 47? 1 S02.

Ommastrephes gigas, d'Orb., Voy. dans l'A mér. Mér., p. 50, p]. 4, 1835.

Ommastrephes giganteus, d'Orb., Céph. Acét., p. 350, 1839.

Ommastrcphes gigas, Tryon, Manual of Conchology, Tol. I, p. 179, pl. s0, figs. $357,360,1879$.

Ommastrephes giga., Hoyle, Report on Cenhalopota, II.MI.s. " ('hallenger." Zoology, Vol. XVI, pp. 32, 214, 1586.

Habitat.-North Queensland Coast; New South Wales; Lord Howe Island. (A.MI.)

\section{Ommastrephes oualaniensis, Tiesson.}

Loligo oualaniensis, Lesson. Toy. de "Conpille," Zool., p. 210, pl. 1, fig. "2, 15:30. Loligo vanikoriensis, Quoy et Gaim., Tof. "Astrolabe," Vol. II, p. 79, pl. 5, figs. $1,2,1832$. 
Loligo brevitentaculuta, Quoy et Gaim., Voy. "Astrolabe," Vol. II., p. 81, 1832. Ommas'rephes oualuniensis, d'()'b., C'éph. Acét.. p. 341; Calamars, pl. 3, pl. 21, figs. 1, 2, 1839 .

Ommastrephes oualaniensis, Stp., Ammot. Blakspr, pp. 76, 84, 1880.

Ommastrephes ounlanirnsis, Hovle, Report on Cephalopoda, II.M.S. "Challenger," Zoology, Vol. XVI, pp. 33, 161, 214, 1886.

Habitat.-Torres Straits, (ireat Barrier Reef, Nickol Bay, North-West Australia. (J. Brazier.)

\section{Ommastrephes Gouldi, McCoy.}

Ommastrephes Gouldi, McCoy, Prodromus of the Zwology of Victoria, Decade, XVII, Vol. II., p. 255, pl. 169, 170, 1888.

Hatitat.-Victorian Waters, Sonth Coast of Australia. (Professor MCCoy.)

Family ONCHII, Steenstrup, 1861.

Sub-family ONYCHO'TEUTHIDE, Gray, 1849.

ONYCHOTEUTHIS, Lichtenstein, 1818.

Onychoteuthis rutilus, Gould.

Onychoteuthis rutilus, Gld., Moll., Wilkes' Exped., p. 1S2, fig. 595, 1852.

Onychoteuthis rutilus, Tryon, Manual of Conchology, Vol. I, p. 169, pl. 7, fig. 302, 1879 .

Onychoteuthis rutilus, Ifoyle, Report on Cephalopoda, H.M.S. "Challenger." Zoology, Vol. XVI, p. 39, 1886.

Habitat.-Near Sydney, New South Wales. (Wilkes' Expedition.)

Family CRANCHIAFORMES, Steenstrup, 1861.

Sub-family CRA NCHIADA, Gray, 1849.

TAONIUS, Steenstrup, 1861.

\section{Taonius Suhmi, Lankester.}

Procutistes suhmii, Lankester, Quint. Jour. Micr. Sei., Tol. XXIT, p. 311. 1884.

Taonius sumii, Hople, in Narrative, "Challenger" Expedition, p. 472, figs. $173,174,1885$.

Taonius suthii, Hoyle, Report on the Cephaloporla, II.MI.S. "Challenger," Zoology, Vol. XVI, pp. 45, 199, pl. 32, figs. 5-11, 1886.

Habitat.-Station 159; Southern Oeean due south of Australia, lat. $47^{\circ}$ $25^{\prime} \mathrm{S}$, long $130^{\circ}$ 2.2' E.; 2,150 fithoms. Between Sydney and Wrellington, surface specimens (H.M.S. "Challenger"). 


\section{Order TETRA BRANCHIATA, 0wen, 1832.}

Family NAUTILID A, Owen, 1836.

NAUTILUS, Linné, 1757.

\section{Nautilus pompilius, Linné.}

Nautilus pompilius, Linn., Syst. Nat., ed. X, 1. 708, No. 293, 232, 1758.

Nautilus major crassus, Martini, Conch. Cab., Tol. I, p, 226, Vignette 10, pl. 18, fig. 164 ; pl. 19, figs. 165, 166, 1769.

Nautilus pompitius, Gmelin, Syst. Nat., Vol. I, part VI, p. 3,369, No.1, 1791. Nautilus pompilius, Lamarek, Anim. sans. Vert., Vol. VII, p. 632, 1 S22.

Nautilus pompilius, Burrow, Elements of Conchology, p1. 12, fig. 2, 1s25.

Nautilus pompitius, Menke, Moll., Noræ Hollandiæ, p. 5, No. 2, 1813.

Nautilus pomnilius, Sowerby, Thes. Conch., Tol. II, p. 463, pl. 97, fig. 1, pl. 99, fig. 6, 1848.

Nautilus pompitius, Hanley, Ipsa Linnæi Conchylia, p. 155, 1855.

Nautilus pompilius, Wood, Index. Test., ed. Hanley, p. 73, pl. 3, fig. 11, 1856. Nautilus pompilius, Reeve, Conch. Icon., Vol. XII, pl. 1, 2, 1861.

Nautilus pompilius, Kuster, Conch. Cab., sec. 55, p. 9, pl. 2, fig. 2, 1 Stis.

Nautilus pompilius, Angas, Proc. Zool. Soc., p. 178, No. 1, 1877.

Nautilus pompilius, Brazier, "Cherert" Exped., \& Shells, Proc. Limn. Soc., Now South Wales, Vol. II, p. 143, No. 1, 1877-1878.

Nautilus pompitius, Tryon, Manual of Conchology, Vol. T, p. 2, pl. 99, figs. $507,508,1879$.

Nautilus pompilius, Hoyle, Report on the Cephalopoda, H.M.S. "Challenger" Zoology, Vol. XVI, pp. 47-199, No. 1, 1886.

Habitat.-Warrior Island, Torres Straits; cast ashore at the Bellinger River, and Coff's Harbour, New South Wales, Congee Bay, south of Syrlney, cast ashore after the great easterly gale of 1857. (J. Brazier.)

\section{Nautilus ambiguus, Sowerby.}

Nautilus ambiguus, Sowerby, Thes. Conch., Vol. II, p. 464, pl. .7, fig. 2, 1848. Nautilus pompitius, Reeve (non Limné) C'onch. Tcon., Vol. XII, species 1, 1861 Nautilus ambiguus, Brazier, "Chevert" Exped. Shells, Proc. Linn. Soc., New South Wales, Vol. II, p. 143, No. 2, 1877-1878.

Nautitius pompitius, Tryon, Manual of Conchology, Vol. I, index, p. 271, 1879 
Habitat.-Cape Gronville, North Queensland, cast ashore on the beaches in rast numbers. (J. Brazier.) This species is thicker, wider at the aperture, nearly all white, with rery few redish-brown radiating stripes. and more depressed round where the umbilicus. should be than in typical Nautilus pompilius, Linné.

\section{Nautilus stenomphalus, Sowerby.}

Nautilus stenomphalus, Sowerby, Thes. Conch., Vol. II, p. 465, pl.97, fig. 3. 1848.

Nautilus stenomphalus, Reeve, Conch. Icon, Vol. XII, pl. 5, fig. 1, 1861.

Nautilus stenomphalus, Kuster. Conch. Cab., sec 55, p. 11, pl. 3b, 1868.

Nautilus stenomphalus, Brazier, "Cherert" Exped. Shells, Proc. Linu. Soc..

New South Wales, Vol. II, p. 144, No. 3, 1877-1878,

Nautilus stenomphalus, Tryon, Manual of Conchology, Vol. II, p. 216, pl. 99, fig. 509, 1879.

Nautitus stenomphalus, Hoyle, Report on the Cephalopoda, H.M.S. "Challenger,"' Zoology, Vol. XVI, p. 47, No. 5, 1886.

Habitat.-Darnley Island, Torres Straits. (J. Brazier.)

\section{Nautilus macromphalus, Sowerby.}

Nautilus macromphatus, Sowerby, Thes. Conch., Vol. II, p. 464, pl. 98, figs. $4,5,1848$.

Nautilus macromphalus, Reeve, Conch. Icon., Vol. XII, pl. 6, fig. 5. 1861.

Nautilus macromphalus, Kuster, Conch. Cab., sec. 55, p. 10, pl. 3a. 1868.

Nautilus macromphalus, Angas, Proc. Zool. Soc., p. 178, 1877.

Nautilus macromphalus, Tryon, Manual of Conchology, Vol. 1, p. 216, pl. 99, fig. 510, 1879.

Nautitus macromphatus, Hovle, Report on the Cephalopoda, H.M.S. " (hallenger," Zoology, Vol. XVI, p. 47, No. 3, 1886.

Habitat.-Cast ashore at the Bellinger River and Coff's Harbour, Yew South Wales, after easterly gales. (J. Brazier, 1870.) 



\title{
AUSTRALIAY MUSEUM, SKMJEI
}

(CATALOGUE No. 15.)

\section{CATALOGUE}

\author{
OF THE
}

\section{MARINE SHELLS OF AUSTRALIA AND TASMANIA.}

\author{
PART II. \\ PTEROPODA.
}

$B Y^{-}$

JOFIJ BEAZIER, O.M.Z.S., F-I.S.

PRINTED BI ORDER OF THE TRUSTEES.

L. P. Pamsay, Curator:

Snomin:

CIIARLES POTTER, GOVERAMENT PIISTEL. 

THis portion of the Catalogue contains the Pteropoda (Hyalia) Families, Limacinidæ, Cavoliniidæ, Notobranchæidæ, and Clionidæ.

The exact habitats are given of those species found by the compiler on the beaches and obtained in the towing net off the New South Wales coast, Torres Straits, and Northern Territory.

It does not appear that any species have ever been recorded from Tasmania, South or Western Australia.

J. BRAZIER. 



\section{PTEROPODA.}

\section{order I.-THECOSOMATA.}

Family I.-LIMACINID A:

Limacina inflata, d'Orbigny.

Attente inflate, d'Orbigny, Toyage dans l'Amérique, Tol. V. p. 174, pl. 12, figs. 16, 19, 1836.

Spivialis rostralis, Eydoux et Souleyet, Description sommaire de plusieurs

Ptéropodes nouveanx ou imparfaitement eonnus, Revue Zoologique, Tol. III, p. $236,18 \pm 0$.

Limacina inflata, Gray Catalogue of the Mollusca in the British Museum, part ii, Pteropoda, p. 31, 1850.

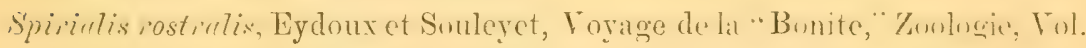
II, p. 216, pl. 13, figs. 1, 10, 1852 .

Jimeinu secephoiden, Genld, The Mollusea and shells of the Luited stater, Exploring Expedition, p. 485, pl. 51, fig. 602, 1852.

Protomeden elate, O. G. Costa, Microdoride mediterranea, p. 74 , pl. 11, fig. 5,1861 .

Embolus rostratis, Jeffreys, Mediterramean MLollusen, Amm, and Mag. Nat. Hist., ser. 4, Tol. VI, p. 86.

Pirotomeden rostralis, Fischer, Sur la faune Malacologique abyssale de la

Méditerranée, Comptes rendus, T'. XCIV, p. 120, 1882.

Limacinu infatu, Pelseneer, Report on the Pteroporti, II.M.s." " "hallenger"," Zoology, Vol. XXIII, p. 16, 1888.

Limacina inflata, Whitelegge, List of the Invertebrate lauma of Port Jackson and Neighbourhood, Journal and Proceedings of the Royal

Society of Now South Wales, Vol. XXIII, p. 280, No. 769, 1889.

Habitat.-Between Sydney and Melboume. (H.M.S. "Challenger"). Brolien Bay, Purt Stephens. ('ongee Bay, New South Wales: fommi in shell sand. (J. Brazier.)

\section{Limacina Lesueuri, d'Orbigny.}

Atlanta Lesucuri, d'Orbigny, Voyage dans l'Amérique méridionale, Tol. V, p. 177, pl. 20, figs. $12,15,1836$.

Atlanta Rangii, d'Orbigny, Toyage dans l'Amérique méridionale, Tol. T, p. 176, pl. 12, figs. $25,28,1836$. 
Spirialis rentricosa, Eydoux et Souleyet, Deseription sommaric de plnsicurs Ptéropodes nouveaux au imparfaitement comnus, Rerue Zoologique, Vol. III, p. 236, 1840.

Limacina ventricosa, Gray, Catalogue of the Mollusen in the British Museum, part II, Pteropoda, p. 32, 1850.

Spirialis ventricosa, Eydoux et Souleyet, Toyage de la "Bonite," Tol. IJ, p. 218 , pl. 13, figs. 11, 16, 1852 .

Spirialis ventricosa, Rang et Souleyet, Hist. Nat. des. Moll., Ptéropodes, p. 63, pl. 14, figs. 13, 18, 1852 .

Limacina Lesueuri, Boas, Spolia Atlantica, p. 46, pl. 3, figs. 33, 34, 1856.

Limacina lesueuri, Pelseneer, Report on the Pteropoda, II.MI.S. "Challenger," Zoology, Vol. XXIII, p. 24, 1888.

Limacina Lesucuri, Whitelegge, List of the Invertebrate Fauma of Port Jackson and Neighbourhood, Journal and Proceedings of the Royal Society of New South Wales, Vol. XXIII, p, 280, No. 770, 1889.

Trabitat.-Between Sydney and Melbourne. (H.M.S. "Challenger"). Broken Bay, Port Stephens, New South Wales; in shell sand. (J. Brazier.)

Limacina trochiformis, d'Orbigny.

Attanta trochiformis, d'Orbigny, Toyage dans l'Amérique méridionale. Vol. V, p. 177, pl. 12, figs. 29, 31, 1836.

Spirialis trochiformis, Eydoux et Souleyet, Deseription sommaire de quelques

Ptéropodes nouveaux ou imparfaitement connus, Rerue Zoologique, Vol. III, p. 237, 1840.

Limacina trochiformis, Gray, Catalogue of the Mrollusea in the British Museum, part II, Pteropoda, p. 33, 1850.

Limacina naticoides, Rang, Itist. Niat. des Moll., Ptéropodes, pl. 10, fig. 1 2, 1852.

Spirialis trochiformis, Eydoux et Sonleyet, Toyage de la "Bonite," Tol. II, p. 223, pl. 13, figs. 27, 31, 1852 .

Spirialis trochiformis, Rang et Souleyet, IIist. Nat. des M[oll., Pteropoder, 1). 64, pl. 14, fig. 27-31.

Limacina trochiformis, Boas, Spolia Atlantica, p. 45, 1856.

Iimacina trochiformis, Pelsencer, Report on the Pteropodar of II.MI.S. "Challenger," Zoology, Vol. XXIII: p. 29, 1898. 
Limacina troctiformis, Whitelegge, List of the Inrertebrate Fauna of l'ort Jackson and Neighbourhood. Journal and Proceedings of the Rovil Society of New South Wales, Tol. XXIII, p. 2S0, No. 771, 1859.

Inatat.-Between Nelbourne and Sydney. (II.M.S. "Challenger".). Broken Bar, Port Stephens, Congee Bay. X'w South Wales; found in shell sand. (J. Brazier.)

\section{Limacina bulimoides, d'Orbigny.}

Atlante bulineides, d'Orbigny, Voyage dans l'Amérique méridionale. Vol. V. p. 179, pl. 12, figs. 36, 38, 1836.

Spiriatis butimoides, Eydoux et Souleyet, Reruc Zoologique, Tul. 1IT, 1. 2:3, 1810.

Limacina butimoides, Gray, Catalogue of the Mollusca in the British MIuseum, part II, Pteropoda, p. 34, 1850.

Spirialis butinoides, Eydoux et Souleyet, Voyage de la "Bonite," Zuologie, Vol. II, p. 224, pl. 13, figs. 35, 42, 1852.

Spirialis bulimoides, Rang et Soulejet, IIist. Nat. des. Moll., Ptéropodes, p. 65, pl. 15, figs. 1, 5, 1852.

Therofusus bulimoides, Angas, Marine Molluscal of Port Jacksom. Prece. Zool. Soc., London, p. 99, 1877.

Limacina butimoides, Boas, Spolia Atlantica, p. 17, pl. 3, figs. 36, 37, 1 SS6.

Limacina bulimoides, Pelseneer, Report on the Pteropoda of II.MI.S. "Challenger," Zoology, Vol. XXIII, p. 31, 1888.

Limacina butimoides, Whitelegge, List of the Invertebrate Fauna of Port Jackson and Neighbourhood, Journal and Proceedings of the Royil Society of New South Wales, Vol. XXIII, p. 280, No. 772, 1889.

ILabitat.-Broken Bay, Port Ntephens, Botany Bay, New South WIales: found in shell sand on beaches. (J. Brizier.) Between Melbonme and sydney in towing net. (H.M.s. "Challenger.")

\section{Family II.CAVOLINIIDE.}

Clio (Creseis) virgula, Rang,

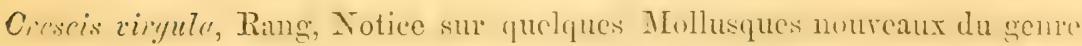
Cleodora, Ann. d Sci. Nat., sér. 1, Tol. XIII, p. 316, pl. 17, fig. 2, 1 s25. Ciescis unguis, Eschscholtz, Koologrischer Atlas. Iteft. 111, p. 17, pl. 15, fir. 4, 1829. 
Cieseis comncopice, Eschscholiz, Zoologischer Atlas, Heft III, p. 17, pl. 15, fig. 5, 1829.

Cireseis caligule, Eschscholtz, Zoologischer Atlas, IIeft III, p. 18, pl. 1.5. fig, 6,1829 .

Hyntee comiformis, d'Orbigny, Toyage dans l'A méricue méridionale, Vul. $\mathrm{V}$, p. 120 , pl. 8, figs. 20, 23, 1836.

Styliole virgnle, Crray, Catalogue of the MLullusea in the British MIuscum. part II, Pteropoda, p. 17, 1850.

Styliola corniformis, Gray, Catalogue of the Mollusea in the British Museum, part II, Pteropoda, p. 17, 1850.

Cleodora virgula, Eydotix et Souleyet, Toyage de la "Bonite," Zoologice, Vol. II, p. 196, pl. 8, figs. 18, 25, 1852 .

Cleodora munda, Gould, Mollusea ant Shells of the United States Exploring Expedition, p. 489, pl. 51, fig. 607, 1852.

Cleodore plucidn, Gould, Mollusea and Shells of the United States Exploring Expedition, p. 489, pl. 51, fig. 606, 1852.

Cleodora falcate, Gould, Mollusea and Shells of the United States Exyloring Expedition, p. 490, pl. 51, fig. 608, 1852.

Creseis virgula, Sowerby, in Reeve, Conch. Icon., Vol XX, Pteropoda, pl. 5, fig. 32, 1877.

Cleodora flexa, Pfeffer, Akad. d. WViss. Berlin, p. 241, figs. 15, 16, 1879.

Creseis virgula, Sowerby, Thes. Conch., Tol. V, Pteropoda, pl. 2, figs. 39, 40, 1884 .

Cleodora virgula, Boas, Spolia Atlantica, pp. 57, 210, pl. 5, fig. 71 ; pl. 6, fig. $94_{;}{ }^{a, 0}, 1886$.

Clio (Crescis) rirgula, Pelsencer, Report on the Pteropoda, II.MI.S. "Challenger," Zoology, Vol. XXIII, p. 48, 1888.

Clio (Creseis) virgula, Whitelegge, List of the Invertebrate Fiuna of P'ort Jackson and Neighbourhood, Journal and Proceedings of the Royal Society of New South. Wales, Vol. XXIII, p. 280, 773. 1889.

Habitat.-Cabbage-tree Bay, or Shallow Beach, Manly Beach, Port Stephens, Broken Bay, New South Wrales; in shell sand after gales. (J. Brazier.) Off. Sydney If adds in towing net; between M[clbourne and sydney. (H.M.S. "Challenger.") 
Clio (Creseis) acicula, Rang.

Cirescis acicula, Rang, Anu. d. Sci. Nat., sér. 1, Vol. XIII, p. 318, pl. 17, firg. 6, 1828.

Creseis clava, Rang, Ann. d. Sci. Nat., sér. 1, Vol. XIII, p. 317, pl. 17, tig. 5,1828 .

Creseis acus, Eschscholtz, Zoologischer Atlas, Heft. III, p. 17, pl. 15, firg. 2, 1829.

Hyalca aciculata, d’Orbigny, Torage dans l’Amérique méridionale, Tol. I, p. 123, pl. 8, figs. $29,31,1536$.

Styliola recta, Gray, Catalogue of the Mollusea in the British Mruseum, part II, Pteropoda, p. 1S, 1850.

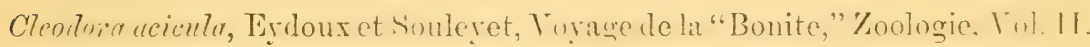
p. 104 , pl. 8, figs. $10,17,1852$.

Cleodora acicula, Rang et Sonleyet, Hist. Nat. des. Moll., Ptéropodes, p. 56 pl. 6 , fig. 7 and 5,1852 .

Crescis aciculata, Sowerby in Reeve, Conch. Icon., Vol. XX, Pteropoda, pl. 5 , fig. 29, 1877 .

Cieseis aciculata, Sowerby, 'Thes. Conch., Vol. V, Pteropoda, pl. 2, fig. $4.5,16$ 1581.

Clcodora acicula, Boas, Spolia Atlantica, p. 59, pl. 6, fig. 94, 1856.

Clio (Ciescis) acicula, Pelseneer, Report on the Pteropoda, H.MI.S. "Challenger," Zoology, Vol. XXIII, p. 4s, 18ss,

Clio (Creseis) acicula, Whitelegge, List of the Invertebrate Fiuma of Port Jackson and Neighbourhood, Journal and Proceedings of the Royal Society of New South Wrales, Vol. XXIII, p. 2\$0, 774, 1589.

Habitat.-Cabbage-tree Bay, or Shal'ow Beach, Manly Beach, Port Stephens, Broken Bay, New South Wales; in shell sand after gales. (J. Brazier.) Off Sydney Heads in towing net; Torres Strails. (II.M.S. "Challenger.")

Clio (Hyalocylix) striata, Rsng.

Cieseis striata, Rang, Ann. d. Sei. Nat., sér. 1, Vo?. XIII, p. 315, pl. 15, tier. $7,1828$.

Creseis compiessa, Eschscholtz, Zoologischer Itlas, Heft. III, p. 17, pl. 15, fig. $7,1829$.

Cireseis zonala, Delle Chiajo, Memoric sulla storia e notomia degli anima!i senza rertebre, pl. 82, fig, 9, 1S:30. 
Styliola stivita, Grar, Catalogue of the Mollusca in the British Muscum, part II, Pteropoda, p. 18, 1850.

Clcodora striata, Rang et Souleyet, Hist. Nat. des. Moll., Ptéropodes, 1) 55 pl. 6. fig. 3, 1852 .

Cleodora striata, Eydoux ct Souleyet, Toyage de la "Bonite," Zoologie, Tol. II, p. 191, pl. 8, figs. 1, 4, 1852 .

Crescis phacostoma, Troschel, Archiv f. Naturgesch, p. 206, pl. S, figs. 5, 7, 1854 .

Creseis striata, Sowerby in Recre, Conch. Icon., Tol. XX, pl. 5, Pteropoda, fig. $30,{ }^{\mathrm{a}, \mathrm{b},} 1877$.

Cicscis striata, Sowerby, Thes. C'onch., Tol. T, Ptcropoda, pl. 2, fig. 3s, 1584. Cleodora striata, Boas, Spolia Atlantica, pp. 62, 202, 1886.

Clio (Iyalocylix) striata, Pelseneer, Report on the Pteropoda of II.M.S. "Challenger," Zoology, Vol. XXIII, p. 54, pl. 2, fig. 3, 1888.

Clio (Iy)locylix) striuta, Whitelegge, List of the Invertebrate Fauna of Port Jackson and Neighbourhood, Journal and Proceedings of the Royal Society of New South Wales, Tol. XXIII, p. 280, No. 775, 1859.

Inabitat.-Cabbage-tree Bay or Shallow Beach, Manly Beach, Port Stepheus, Broken Bay, New South Wales; in shell sand after gales; Torres Straits; Port Darwin. (J. Brazicr.) Raine Island, near Torres Straits; 135 fathoms. (H.M.S. "Challenger.")

Clio (Styliola) subula, Quoy and Gaimard.

Styliote recta, Lesueur, in de Blainville, Mannel de Malacologie, 1. 655, 1825.

Cleodora subuta, Quoy et Gaimard, Obserrations Zoologiques faites it hord de "l'Astrolabe," Ann. d. Sci. Nat, sér. 1, Vol. X, p. 223, pl, 8, D. figs. $1,3,1827$.

Creseis spinifera, Rang, Ann. d. Sei. Nat., sér. 1, Tol. XIII, p. 313, pl. 17, fig. 1, 1828.

Crescis subula, Rang, Aun. d. Sei. Nat., sér. 1, Tol. XXIII, p. 313, pl. 17, fig. $1,1828$.

Iryatar subula, d'Orbigny, Toyage dans l'Amérique, Tol. V, p. 119, pl. S, figs. $15,19,1836$.

Styliola subulte, Grals, Catalogue of the Mrollusea in the British MInseum, part II., P'teropoda, p. 17, 1850.

Cleodorn subulatn, Rang et Sonleyet, Ifist. Nat. des. Moll., Ptéropodes, p. 5.5. pl. 6, fig, 1, 1852 . 
Cleodora subulatr, Eydoux et Souleyet, Toyage de la "Bonite," Zoologie, Tol. II, p. 192, pl. 8, fis. 5, 9, 1852.

Styliola subulata, Angas, Marine Mollusca of Port Jackson, Proc. Zool. Soc. London, p. 178, 1877.

Crescis spinifera, Somerby in Reeve, Conch. Icon., Tol. XX, Pteropoda, pl. 5, fig. $33,1877$.

Cieseis spinifera, Sowerby, Thes. Conch., Tol. V, Pteropoda, pl. 2, figr.. 43, $44,1884$.

Cleodora su7 $u^{7} a$, Boas, Spolia Allantica, rp. (55, 202, pl. 1, firs. 11, 4.5, 1586.

Ciio (Styliola) subula, Pelieneer, Report on the Pteropoda of H.MI.S. "Challenger," Zoology, Tol. XXIII, p. 57, 1888,

Habitat.-Cabbage-tree Bay, or Snallow Beach, Manly Beach, Port Stephens, Brolien Bay, Bellinger Rirer, Now South Wales; in shell sand after gales; Port Darwin. (J. Brazier.) Off Sydney and Melbourne, in towing net; Raine Island, near Torres Straits. (H.M.S. "Challenger,")

\section{Clio pyramidata, Linné.}

Clio pyjamidata, Linné, Systema Naturæ, ed. 12, p. 1091, 1767.

Myalér lanceolala, Lesueur, Mémoire sur quelques espèses d'animanx mollusques et radiaires recueillis dans la Méditerranée près de Nice, Nouv. Bull. Soc., Philom.; Paris, Vol. III, p. 28t, pl. 5, fig. 3, 1813.

Cleodora Brownii, De Blimville, Manuel de Malacologie, pl. XLVJ, fig. 1, 1825.

IFyalea pyramidata, d'Orbigriy, Toyage dans l'Imérique méridionale, Tul. T, p. 113, pl. 7, figs. 25, 29, 1 S36.

Clcodora Lamartinieri, Rang, in d'Orbigny, MIo'lusques de Cuba, p. \$3, 1841. Clio pyramilata, Gray, Catalegue of the MIollusea in the British Minemu, part 1I., Pteropoda, p. 12, 1850.

Clcodora lanceolat", Eydux et Siouleyet, Toyane de la "Bonite," Zuolacice, Tol. V, p. 179, pl. 6, figs. 17, 25, 1852.

Clecdora pyramidula, Rangr et Sinnleyet, Hist. Nat. Iles MFoll., Ftírupoder, p. 50, pl. 5, fig. 11, figs. $7,10,1852$.

Cleodore exacula, Gould, The Mollusea and Shells of the L.S. Expluringr Expedition, p. 485, pl. 51, fig. $605,1852$.

Cleodora lobata, Sowerby, in Reeve, Conch. Teon., Tol. XX, Pteropoda, fig. 26,1877 . 
Cleodora martensii, Pfeffer, Du Pteropoden des IJamburger Muscums,

Abhandl. d. Naturw. Ver, Hamburg, Bd. VII, p. 95, pl. 7, fig. 16, 1880. Cleovora pyramidata, Boas, Spolia A tlantica, p. 69, pl. 6, figs. 96-97, 1836 .

rlio pyiramidata, Pelsencer, Report on the Pteroporla of II.M.S. "Chailenger," Vol. XXIII, p. 63, 1888.

Habitat.-Cabbage-tree Bay, or Shallow Beach, Manly Beach; Port Stephens, Broken Bay, New South Wales; in shell sand after gales. (J. Brazier.) Off Sydney Ileads, 950 fathoms, bottom gren mud; off Melbourne, in towing net; Raine Island, near Torres Straits. (H M.S. "Challenger.")

\section{Cuvierina columnella, Rang.}

Cleodora obtusa, Quoy et Gaimard, Voyage de 1' "Uranie," Zoologio, p. 415, pl. 66 , fig. 5, 1824.

Cuvieria columnella, Rang, Ann. des Se. Nat., Tol. XII, p. 323. pl. 45, figs. $1,8,1827$.

C'urieria oryza, Benson, Corrected characters of the genus Cuvieria and notice of a second species inhabiting the tropical Indian Ocean, Journal Asiat., Soc. Bengal, Vol. IV, p. 698, 1835.

Tiptere columella, Gray, Catalogne of the Mullusca in the British Mruseum, part II, Pteropoda, p. 23, 1850.

Cuvieria urceolaris, Mörch, Catalogus conchyliorum quae reliquit, C. P. Kjerulf, p. 32, 1850.

Cuvieria columnella, Eydoux et Souleyet, Toyage de la "Bonite," Tol. 11, p. 205, pl. 12, figs. 1, 11, 1552 .

Cuvieria columnella, Rang et Soulejet, Hist. Nat. des Moll. Ptéropodes, p. 59, pl. 4, pl. 14, figs. 1, 6, 1852.

Triptera columnella, Angas, Marine Mullusea of Port Jackison, Proe. Zonl. Soc., London, p. 179, 1877.

Triptera columnella, Sowerby in Recve, Conch. Tcon., Vol. XX, Pterupoda pl. 5, fig. 27, 1877.

Tipteia columne7la, Pfefter, Monatsber. d. k. preuss. Akal. 九. Wiss, Berlin, p. 213, fig. 18, 1879.

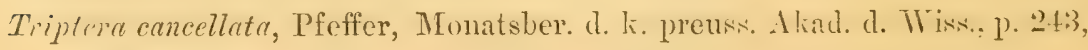
fig. 19, 1879.

Tiiptera columnella, Sowerby, Thes. Conch., Tol. V, p. 145, Pteropoda, pl. "2, fig. 49, 1881 .

Cuvierina columnella, Boas, Spolia Atlartica, pp. 131, 133, pl. 3, fig. 39, pl. 6, fig. $95,1886$. 
Gevierina columella, Pelseneer, Report on the Pteroporla of II.MI.s. "('hallenger," Zoology, Vol. XXIII, p. 67, 1888.

C'uvierina columnella, Whitelegge, List of the Invertebrate Fauma of Port Jackson and Teighbourhood, Journal and Proceedings of the Royal Society of New South Wales, Vol. XXIII, p. 280, No. 778, 1859.

Habitat.-Cabbage-tree Bay, or Shallow Beach, Manly Beach; Port Stephens, Broken Bay, New South Wales. (A.M.) In shell sand on the beaches after gales. Off Sydney Heads, in towing net; Port Darwin. (J. Brazier.) (A.MI.) Raine Island, near Torres Straits. (II.M.S. "Challenger.")

Cavolinia trispinosa, Lesueur.

Anomia aculeata major, Chemnitz, Conch. Cab., Vol. VIII, Vignette, plate 13, p. 65, letter a, b, Pp. 71, 92, 1785.

Anomia aculeata maxima, ('hemnitz, Conch. Cab., Tol. VIII, Vignette, plate 13, p. 65, letters c, d, pp. 71, 93, 1785.

Myalae trispinosa, Lesueur, MS. in de Blainville, Hyale, Dict. d. Mci. Nat., Vol. XXII, p. 82, 1821.

IIyalcea mucronata, Quoy et Gaimard, Observations Zoologiques faiter it bord de l' "Astrolabe," Ann. d. Sci. Nat., sér. 1, Vol. X, p. 231, pl. 8B, figs. 1, 2, 1827.

Hyalca depressa, Bivona, Descrizione di una nuora specie di Jale, Efemeridi scientifiche e litterarie per la Sicilia, p. 57, pl. 1, figs. 4, 5, 1832.

IIyalar cuspilata, Delle Chiaje, Descrizione notomia degli animali senza vertebre del Regno di Napoli, pl. 1so, figs. 1, 2 (non d'Orbigny), $18+1$.

Diacria trispinosa, Gray, Catalogue of the Mollusea in the British Museum, part II, Pteropoda, p. 10, 1850.

Diacria mucronata, Gray, Catalogue of the Mollusea in the British Muscum, part II, Pteropoda, p. 11, 1550.

Hyalea trispinosa, Eydoux et Sonleyet, Toyage de la "Bonite," Zoulogre, Vol. II, p. 161, pl. 6, figures 1, 10, 1862.

IYyalea trispinosa, Rang et Souleyet, Hist. Nat. des Moll. Ptéropude's, p. 45, pl. 3, figs. 1, 7, 1852.

Hyalå Reeviana, Dunker, Index Molluscorum, p. 2, pl. 1, figs. 17, 20, 1853.

Pleuropus trispinosus, II. and A. Adams, Genera of Recent Molluscil, Vul. II, p. 611, 1858.

Plenropus mucionatus. II. and A. Adams, Genera of Recont Mollusea, Tol. II, p. $611,1858$. 
Diacria mucronata, Angas, Marine Mollusea of Port Jackson, Proc. Zool. Soc., London, p. 17S, 1877.

IIyalea (Diacria) trispinosa, Sowerby in Reeve, Conch. Icon., Tol. XY, Pteropoda, pl. 3, fig. 15, 1877.

Hyalcea (Diacria) mucronata, Sowerby in Reere, Conch. Ieon., Tol. XI, Pteropoda, pl. 3, fig. 16, 1877.

Myalcea (Diacria) trispinos, Sowerby, Thes. Conch., Tol. V, p. 111, Pteropoda, pl. 1, fig. 20, 1854.

Hyalcea (Diacria) mucronata, Sowerby, Thes. Cunch., Tol. V, p. 112, Pteropoda, pl. 1, figs. 27, 28, 1834 .

Iyclea trispinosa, Boas, Spolia Atlantica, p. 9t, pl. 1, fig. 3; pl. 2, fig. $14,1856$.

Iyalea trispinosa, Sowerby, Illustrated Indes of British Shells, sesond edition, pl. 10, fig. 1, 1887.

Civolinia trispinosa, Pelseneer, Report on the Pteropoda, H.MI.S. "Challenger," Zoology, Vol. XXIII, pp. 76, 77, $18 s 8$.

Curotina trispinosa, Whitelegge, List of the Invertebrate Fauna of Port Jaclison and Neighbourhood, Journal and Proceedings of the Rojal Society of New South Wales, Vol. XXIII, p. 280, No. 779, 1889.

Habitat.-Cabbage-tree Bay, or Shallow Beach, Manly Beach, Purt Stephens, Broken Bay, New South Wales, off sydney IIeads and Bondi Bay in towing net; Torres Straits and inside the Great Barrier Reef in towing net; Port Darwin, in beach sand. (J. Brazier). Off Sydney, 950 fathoms, bottom green sand. (H.M.S. "Challenger.")

\section{Cavolinia quadridentata, Lesueur.}

IIyulea qualridentata, Lesueur, MS., in Blainville, Hyale, Diet. d. Sci. Nat. Vol. XXU, p. 81, 1 S21.

IIyatce quadrispinosa, d'Orbigny, Toyage dans l'Amérique méridionale, Vol. V, p. 85, 1836.

C'urotina quadidentatu, Gray, Catalogue of the Mollusea in the British Museum, part II., Pteropoda, p. 8, 1850.

Hyatce quadidentata, Eydoux et Souleyet, Toyage de la "Bonite," Zoologie, Vol. II, p. 147, pl. 4, figs. 25, 32, 1852.

Hyataa quadridentuta, Rang et Souleyet, Hist. Nat. des Moll., Ptúropodes, p. 39, pl. 3 , figs. $13,15,1852$.

Hyalea inemis, Gould, The Mollusea and Shells of the United States Exploring Expedition, pl. 51, fig. 601, 1852. 
IIyalce minuta, Sowerby in Reere, Conch. Icon., Tul. XX, Ptoropodi, pl. 2, fig. $9,1877$.

Iryalca intermedia, Sowerby in Reere, Conch. Icon., Tol. XX, Pteroporla, pl. 2, fig. 10, 1877.

Carotina quadridentata, Brazier", "Cherert" Expedition. Shells, Proc. Limn. Soc., New South Wales, Vol. II, p. 144, No. 4, 1877, 1878.

Carolina quadridentata, Angas, Marine MLollusea of Purt Jackson, Proe. Zool, Soc, London, p. 178, 1877.

Myalea quulridentutu, Sowerby in Reere, Conch. Icon., Tol. XX; pl. 22, ling. $14,1877$.

Myalca costata, Pfefler, MLonatsber. d. k. preuss. Akad. d. Wiss. Berlin, p. 234, 1879.

Iryatea quadridentata, Sowerby, Thes. ('onch., Tol. V, p. 110, pl. 1, figs. 17 $18,1884$.

Hyala intormedia, Sowerby, Thes. Conch., Vul. V, p. 1н1, Pteropoda, pl. 1, fig. 11, 188 1.

IIyalcea minuta, Sowerby, Thes. Conch., Tol. T, p. 111, Pteroporla, pl. 1, tige. $19,1581$.

Hyalcea quadridentata, Bons, Spolia Atlantica, p. 99, pl. 1, fig. 4; pl. 2, fig. 15, 1886.

Cavolina quadridentata, Pelseneer, Report on the Pteropoda of II.II.S. "Challenger," Zoology, Vol. XXIIT, pp. 78, 79, 1888.

Carolina quadridentata, Whitelegge, List of the Invertebrate Fauna of Port Jackson and Neighbourhood; Journal and Proceedings of the Royal Society of New South Wales, Vol. XXIII, p. 280, No. 780, 1859.

Ilabitat.-Cabbage-tree Bay, or Shallow Beach, Manly Beach, Port Tackison, Port Stephens. Broken Bay, New South Wales; Port Darwin. Darniey Island, Torres Straits, 30 fathoms; one specimen found. (J. Brazier.) (A.M.) Raine Island, near Torres Straits. II.M.S. "('hallenger."

Cavolinia longirostris, Lesueur.

IIyalica Tongirostris, Lesueur, MS., in de Blainsille, Ilyale, Diet. d. Sei. Nat., Vol. XXII, p. 81, 1821.

Hyaïca ecaudata, Lesueur, Dict. d. Sci. Nat., Vol. XXII, p. 82, 1821.

Hyalce limbata, d'Obigny, Toyage daus i’Amérique méridonale, Tol. V, p. 101, pl. 6, figs. 11, 15, 1836.

Carolina longirestra, Gray, Catalogue of the Mollusea in the British Museum, part II, Pteropoda, p. S, 1850. 
IIyalcea angutata, Eydoux et Souleyet, Forage de la "Bonite," Zoologie, Vol. II, p. 152, pl. 5, figs. 1, 6, 15.52.

II!nlin Tongirostris, Egdoux et Souleyet, Vurage de lat "Bonite," Zoulogie, Tol. II, p. 149, pl. 5, figs. 7, 13, 185 ?.

Iryaleu longirostris, Rang et Souleyet, Ifist. Nat. des Moll., Ptéropodes, p. 1 , pl. 2, figs. $7,10,1852$.

IIycrele femorta, Gould, The Mollusea and shells of the L'nited States Exploring Expedition, pl. 5l, fig. 603, 1852.

IIylulen fissirostris, Benson, Notes on the Pteropodous genus Ifyaliea ant deseription of a new species, Ann. and Mag. Nat. Hist. series 3, Vol. VII, p. 26, 1861 .

Curolina longirostris, Angas, Marine Molluseat of Port Jackson, Proc. Zool. Soc., London, p. 178, 1877,

IIyalce obtusa, Sowerby in Reeve, Conch. Icon., Vol. XX, Pteropoda, pl. 2, fig. 8, 1877.

Hyalea longirostris, Sowerby in Reere, Conch. Icon., Vol. XX, Pteropoda, pl. 2, fig. 12, 1877 .

Hyalca limbata, Sowerby in Reeve, Coneh. Ieon., Vol. XX, Pteropoda, pl. 2, fig. 11, 1877.

Hyalea limbata, Sorrerby Thes. Conch. Vol. V, p. 141, Pteropoda, pl. 1, figs. $14,15,1877$.

IIyalce longirostris, Sowerby, Thes. Conch., Vol. V, p. 141, Pteropoda, pl. 1, fig. 16, 1877.

Carolina longirostva, Brazier, "Chevert" Expedition Shells, Proc. Limn. Soc., New South Wales, Vol. II, p. 144, No. 5, 1877, 1878.

IIylece iongivostris, Boas, Spolia Atlintica, p. 102, pl. 1, fig. 5; pl. 2, fig. 16,1885 .

Carolinia longirostris, Pelseneer, Report on the Pteropoda of II.ML.S. "Challenger," Zoology, Vol. XXIII, p. 79, 1888.

Curolina longirostris, Whitelegge, List of the Invertebrate Fauna of Port Jackison and Neighbourhood, Journal and Proceedings of the Royal Siociety of New South Wales, Tol. XXIII, p. 280, No. 781, 1899.

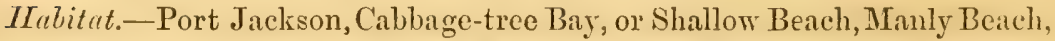
Port Stephens, Broken Bay, New South Wales; Fitzroy Islaud, Princess ('harlotte Bay, North-east Australia, 13 fathoms; Darnley Island, Torres Straits, 30 fathous. (J. Brazier). (A.MI.) Melbourne to Sydney, Raine Island, near Torres Straits. (II.M.S. "Challenger.") 


\section{Cavolinia globulosa, Rang.}

Carolina globulosa, Rang, MS., in Gray, Catalogue of the Mollusea in the British Museum, part II, Pteropoda, p. 8, 1850.

Cavolina pisum, Mörch, Catalogus conchyliorum quae reliquit C. P. Kjerulf, p. 32, pl. 1, fig. 7, 1850.

Hyalan globulosa, Rang, His. Nat. des Moll., Ptéropodes, p. 39, pl. 2, figg. $15,18,1852$.

Hyalaa globulosa, Eydoux et Sonleyet, Voyage de la "Bonite," Zoologie, Vol. II, p. 142, pl. 4, figs. 20, 24, 1852.

Hyalca globulosa, Sowerby in Reere, Conch. Icon, Vol. XX, Pteropoda, pl. 1 , fig. 6, 1877.

Hyalca globulosa, Pfeffer, 1. Monatwb. Berlin Akad., d. Wiss. Berlin, p. 232, 1879.

Hyalca ,llobulosa, Sowerby, Thes. Conch., Tol. V, p. 140, Pteropoda, pil. 1, figs. $8,9,1884$.

Hyallaea globulosa, Boas, Spoliat Atlantica, 1) 107, pl. 1, fig. 7; pl. 2, fig. $18,1886$.

Cavolinia globulosa, Pelseneer, Report on the Pteropoda of H.M.S. "Challenger," Zoology, Vol. XXIII, p. 81, 1888.

Iabitat.-Port Stephens, Port Jackson, New South Wales. (J. Brazier.)

\section{Cavolinia gibbosa, Rang.}

Hyalaa gibbosa, Rang., MS., in d'Orbigny, Toyage dans l'Amérique méridionale, Vol. V, p. 95, pl. 5. figs. 16, 20, 1836.

Hyalca flava, d'Orbigny, Toyage dans l'Amérique méridionale, Tol. T, p. $97, \mathrm{pl} .5$, figs. $21,25,1836$.

Cavolina gibbosa, Gray, Catalogue of the Mollusca in the British Museum, part II, Pteropoda, p. 7, 1850.

Cavolina Alava, Gray, Catalogue of the Mollusca in the British Museum, part II, Pteropoda, p. 8, 1850.

Hyalcea gibbosa, Rang, et Souieyet, Hist. Nat. des Moll., Ptéropodes, p. 38, pl. 10, figs. 3, 4, 1852 .

Hyalca gibbosa, Elyoux et Souleyet, Toyage de la "Bonite," Vol. II, p. 144, pl. 4, figs. 13, 19, 1852.

Hyalaen gibbosa, Sowerby in Reere, Conch. Icon., Vol. XX, Pteropoda, pl. 1, fig. $7,1887$. 
Cavolina gibbosa, Angas, Marine Mollusca of Port Jackson, Proc. Zool. Soc., London, p. 178, 1877.

Hyalea gegenbauri, Pfeffer, Die Pteropoden der Mamburger Museums,

Abhandl. Naturw. Ver. Hamburg, Bd. VII, p. 86, figs. 5, 7, 1880.

Hyalcea gibbosa, Sowerby, Thes. Coneh., Vol. XX, p. 140, Pteropoda, pl. 1, figs. $1,2,1884$.

Hyalcea gibbosa, Boas, Spjlia Atlantica, p. 109, pl. 1, fig. 6; pl. ㄹ, fig. 17, 1886.

Cavolinia giblosa, Pelseneer, Repert on the Pteropoda of II.MI.S. "Challenger," Zoology, Vol. XXIII, p. 82, $18 s 8$.

Carolinia gibbosa, Whitelegge, List of the Invertebrate Fauna of Port Jackson and Neighbourhood, Journal and Proceedings of the Roral Society of New South Wales, Vol. XXIII, p. 281, No. 783, 1889.

Habitat.-Cabbage-tree Bay, or Shallow Beach, Mauly Beach, Port Stephens, Broken Bay, New South Wales; Port Darwin, North Australia. (J. Brazier.) Raine Island, near Torres Straits. (II.M.S. "Challenger.")

\section{Cavolinia tridentata, Forskål.}

Anomia tridentata, Forskil, Deseriptiones animalium quae in itincre orientali observavit, p. 124, 1773.

Anomia aurita scarabaeiformis, Chemnitz, Conch. Cab., Tol. VIII, p. 65, Vignette, pl. 13, fig. G, pp. 72, 108, 1785.

Anomia tridentata, Gmelin, Syst. Nat. Vol. 1, part VI, p. 3318, No.42, 1790.

Cuvolinia natans, Abildgaard, Nyere Efterretnig om det Skaldyr som Forskìhar beskrevet under Navnet Anomia tridentata, Skirr. naturhist. Selsk. Bd. I, Heft. 2, pl, 10, 1791.

Hyalaa cornea, Lamarek, A nim. sans. Vert., p. 140, 1801.

Hyalaa papilionacea, Bory de St. Vincent, Voyage dans les quatre principales îles des mers d'Afrique, Vol. I, p. 137, pl. 5, fig. 1, 1804.

Hyalca Peroni, Lesueur, Mémoire sur quelques animaux mollusques, de., Nouv. Bull. Soc. Philom.; Vol. III, p. 284, 1813.

Hyalaa Chemnitziana. Lesueur, Nour. Bull. Soc. Philom., Tol. III, p. 2S 4 , 1813.

Hyalaca Australis, Peron, Toyage de découvertes aux terres australies, Vol. I, pl. 31, fig. 5, 1816 .

Hyalax tridentata, Lamarck, Anim. sans Tert, Vol. VI, part I, p. 286, I819 
IIyalaa Forskahtii, Lesueur, MS., in de Blinnville, Hyale, Diet. d. Sei. Nat. Vol. XXII, p. 79, 1821.

Hyalee affinis, d'Orbigny, Toyage dans l'Amérique méridionale, Tol. T., p. 91, pl. 3, figs. $6,10,1836$.

IIyalaa tridentata, Lamarek (ed Deshayes), Vol. VII, p. 415, 1836.

Hyalcea truncata, Krauss, Südafricanisehe Mollusken, p. 3t, pl. 2, fig. 12 (non Lesueur), 1848.

Cavolina tridentata, Gray, Catalogue of the Mollusea in the the British Museum, part II, Pteropoda, 1. 6, No. 1, 1850.

Iryalcea tridentate, Eydoux et Souleyet, Voyage de la "Bonite," Zoologie, Vol. IV, p. 137 , pl. 4, figs. 1 to 7,1852 .

IIyalca tridentata, Rang et Souleyet, IFist. Nat. de Mroll., Ptéropodes, p. 35, pl. 2, figs. 1, 5, 6, pl. 12 ; figs. 1, 4, 1852.

Cuvolinia telemus, A. Adams, On the Synonyms and Habitats of Carolinia, Diacria, and Pleuropus, Ann. and Mag. Nat. Hist., Series 3, Tol. IIJ, p. 44, 1859.

IIyalcen affinis, Sowerby in Reeve, Conch. Icon., Vol. XX, Pteropoda, pl. 1, fig. $3,1877$.

Hyalca tridentata, Sowerby in Reeve, Conch. Icon., Vol. XX, Pteropoda, pl. 1, fig. 4, 1877.

Hyalcea Cumingii, Sowerby in Reere, Conch. Icon., Vol. XX, Pteropoda, pl. 1, fig. $5,1877$.

IIyalce Cumingi, Nowerby, Thes. Conch., Vol. V, Pteropoda, pl.1, fig. 4, 1584. Iy yalce tridentata, Sowerby, Thes. C'onch., Tol. T, Pteropoda, pl. 1, fig. 5, 1854.

IIyalac affinis, Sowerby, Thes. Conch., Vol. V, Pteropoda, pl. 1, fig. 10, 1881. Hyalcea tridentata, Boas, Spolia Atlantica, p. 115, pl. 1, fig. S; pl. 2, fig. 19, p. $211,1586$.

Cavolinia tridentata, Pelseneer, Report on the Pteropoda of H.M.'S. "Challenger," Zoology, Tol. XXIII, p. 83, 1888.

Inabitat.-Broken Bay, Port Stephens (A.M.); Bellinger Rirer, New South Wales; beaches after stormy weather. (J. Brazier).

Cavolinia uncinata, Rang.

Hyalca uncinata, Rang, Ms., in d'Orbigny, Voyage dans l'Amerique méridionale, Tol, V, p. 93, pl. 5, figs. 11, 15, 1836.

Cavolina uncinata, Gray, Catalogue of the Mollusea in the British MLuseum, part II, Pteropoda, p. 7, 1850. 
Hyalaa uncinata, Eydoux et Souleyet, Voyage de la "Bonite," Vol. II, p. 140 , pl. 4, figs. 8, 12, 1852 .

Hyalaea uncinata, Rang et Souleyet, Hist. Nat. des Moll., Ptéropodes, p. 37, pl. 2. figs. 11, 14, 1852.

Hyalcea uncinata, Sowerby in Reeve, Conch. Icon., Vol. XX, Pteropoda, pl. 1, fig. 1, 1877.

Hyalca uncinatiformis, Pfeffer, Die Pteropoden des Hamburger Museums Abhandl. Naturw. Ver. Hamburg, Bd. 7, p. 85, 1880.

Hyalca uncinata, Sowerby, Thes. Conch., Vol. V, Pteropoda, pl. 1, figs. 6, 7, 1884.

Hyalaa uncinata, Boas, Spolia Atlantica, p. 119, pl. 1, fig. 10; pl. 2, fig. $20,1886$.

Cavolinia uncinata, Pelseneer, Report on the Pteropoda of H.M.S. "Challenger," Zoology, Vol. XXIII, p. 84, 1888.

Habitat.-Port Jackson (A.M.), Cabbage-tree Bay, or Shallow Beach, Manly Beach, Port Stephens, New South Wales; Port Darwin, North Australia. (J. Brazier); Raine Island, near Torres Straits. (H.MI.S. "Challenger.")

\section{Cavolinia inflexa, Lesueur.}

Hyalaa inflexa, Lesueur, Mémoire sur quelques animaux mollusques, Nour. Bull. Soc. Philom., Vol. III, p. 285, pl. 5, fig. 3, 1813.

Hyalca elongata, Lesueur, MS., in de Blainville, Hyale, Dict. d. Sci. Nat., Vol. XXII, p. 82, 1821.

Hyalca vaginellina, Cantraine, Bull. Acad. d. Se., Bruxelles, Tol. II, p. 350, 1885.

Hyalca labiata, d'Orbigny, Voyage dans l'Amérique méridionale, Vol. $\mathrm{Y}$, p. 104, pl. 6, figs. 21, 25, 1836.

Hyalaa uncinata, Hœninghans, MS., in Phillippi, Enumeratio Molluseorum utriusque Sicilia, p. 101, pl. 6, fig. 18 (non Rang), 1836.

Cavolina inflexa, Gray, Catalogue of the Mollusca in the British Museum, part 2, Pteropoda, p. 9, 1850.

Hyalaa inflexa, Eydoux et Souleyet, Toyage de la "Bonite," Vol. II, p. 156, pl. 5, figs. 21, 26, 1852.

Hyalea inflexa, Rang et Souleyet, Hist. Nat. des Moll., Ptéropodes, p. 44, pl. 3, figs. 9, 10, 12, 1852.

Hyalca labiata, Eydoux et Souleyet, Voyage de la "Bonite," Tol. II, p. 159, pl. 5, figs. 27, 32, 1852 . 
IIyalaa labiata, Rang et Souleyet, Hist. Nat. des M[oll., Ptéropodes, p. 4:3, pl. 12, figs. $15,19,1852$.

Cavolina inflexa, Angas, Mrolluscan Fauna of Port Jackson, Proc. Zool. Soc.. p. $178,1877$.

Hyalaca inflexa (Diacria), Sowerby in Reeve, Conch. Icon., Vol. XX, Pteropoda, pl. 3, fig. $17^{\mathrm{a}, \mathrm{b}}$, 1877 .

Hyalca labiata (Diacria), Sowerby in Reeve, Conch. Icon., Vol. XX, Pteropoda, pl. 3, fig. $18^{\mathrm{a}, \mathrm{b}}, 1877$.

Hyalca imitans, Pfeffer, Die Pteropoden des IIamburger Museums, Abhandl.

Naturw. Ver. Hamburg, Bd. 7, p. 90, pl. 7, fig. $9^{2}, 1880$.

Cleodora inflexa, Sowerby, Thes. ('onch., Tol. V, Pteropeda, pl. 1, figm. .21, 22,1884 .

Diacria labiata, Sowerby, Thes. Conch., Tol. V, Pteropoda, pl. 1, figs. 20?, 24,1884 .

Hyalca inflexa, Boas, Spolia Atlantica, p. 123, pl. 1. fig. 11 ; pl. 22, tig. 221. 1886.

Cavolinia inflexa, Pelsenecr, Report on the Pteropoda of H.M.s. "Challenger," Zoology, Vol. XXIII, p. 85, 1888.

Cavolina inflexa, Whitelegge, List of the Invertebrate Famna of Port Jackson and Neighbourhood; Journal and Proceedings of the Royal Society of New South Wales, Vol. XXIII, p. 281, No. 78t, 1889.

IIabitat.-Port Jackson, Broken Bay, Port Stephens, New South Wales: Port Darwin, North Australia, and in towing net, Torres Straits. (J. Brazier). Raine Island, near Torres Straits. (H.M.S. "Challenger").

\section{Cleodora compressa, Souleyet.}

Clio depressa, Gray, Catalogue of the Mollusea in the British Museum, part II, Pteropoda, p. 14, No. 14, 850.

Cleodora compressa, Eydoux et Souleyet, Voyage de la "Bonite," Zoologie, Vol. II, p. 181, pl. 6, figs. 26, 32, 1852.

Cleodora compressa, Rang et Souleyet, Hist. Nat. des MIoll., Ptéropodes. p. 51, pl. 12, figs. 20, 25, 1852.

Cleodora compressa, Boas, ,polial Atlantica, p. 85, pl. 4 , fig. 5s, bis ; pl. 1. fig. 50, bis, 1886 .

Cleatora compressa, Pelseneer, Report on the Pteropodia of H.MI.s." Challenger," Zoology, Vol. XXIII, p. 87, 1888. 
Cleodorir compressa, Whitelegge, List of the Invertebrate Fauna of Port Jackson and Neighbourhood, Journal and Proceedings of the Royal Society of New South Wales, Vol. XXIII, p. 281, 1889.

IIabitat.-Cabbage-tree Bav, or Shallow Beach, Manly Beach, New South Wales; Port Darwin. (J. Brazier) ; Melbourne to Sydney ; Sydney to Raine Island, near Torres Straits. (H.M.S. "Challenger.")

\section{Order II.-GYINOSOMATA.}

\section{Family III.-NOTOBRANCHEID Æ.}

Notobranchæa inopinata, Pelseneer.

Notoliranchea inopinata, Pelseneer, Report on the Pteropoda of M.M.S. "Challenger," Zoology, Vol. XIX, p. 40, pl. 3, fig. 5, 6, 1887.

Notubranchea inopinata, Whitelegge, List of the Invertebrate Fauma of Port Jackson and Neighbourhood, Journal and Proceedings of the Roral Society of New South Wales, Vol. XXIII, p. 280, No. 767, 1889.

IIabitat.-Off Port Jackson Heads, New South Wales. (Dr. Macdonalı.)

\section{Family IV.-CLIONIDA.}

Clione caudata, Macdonald.

Cliona coudata, Macdonald, Trans. Royal Society, Edinburgh, Vol. XXIIT, p. 185, pl. 9, fig. 3 (non C. caudata, Gray).

Cliona caudata, Whitelegge, List of the Inrertebrate Famma of Port Jackson and Neighbourhood, Journal and Proceedings of the Royal Society of of New South Wales, Vol. XXIII, p. 280, No. 768, 1859.

Habitat. - Off Port Jackson Heads, New South Wales. (Dr. Macdonalu.) 


\section{AUSTRALIAN MUSEUM, SYINEY. \\ (CATALOGUE No. 15.)}

\section{CATALOGUE}

OF THE

\section{MARINE SHELLS OF AUSTRALIA AND TASMANIA.}

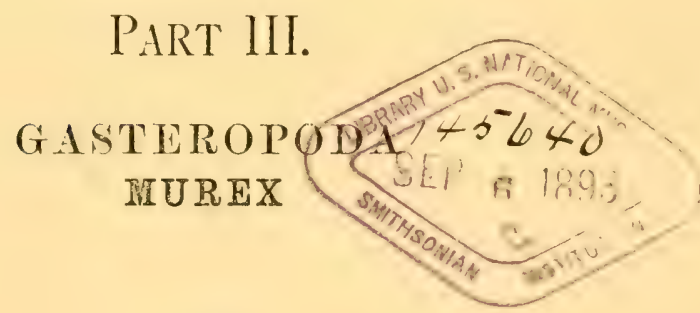

$\mathrm{BY}$

JOFIIT BRAZIER, C.M.Z.S., F.I.S.

PRINTED BY ORDER OF THE TRUSTEES.

E. P. Ramsay, Curator.

Sulner :

CIIARLES POTTER, GOVERNMENT PRINTER. 
Tine present Part contains only the Genus Murex. The remaining gene: a are not inclucled, from causes beyond my control.

June, I 893.

JOHN BRAZIER. 


\section{Sub-class GASTEROPODA, Cuvier, i798.}

\section{Orier PROSORBRANULATA, Mine-Elwards, 1815.}

\section{Family MURICID E, Fleming, 1828.}

MTREX, Linnć, 1758; Purpura, Rondelet, 155.5; Tournefort, 1742;

Arnea, Perry, 1811.

Murex tribulus, Linné.

MIurex tributus, Limné, Syst. Nat., 10 edit., p. 746, No.44t, 1758. Syst. Nat., 12 edit., p. 1214, No. 519, 1766 ; Martini, Conch. Cab., Vol. III, pl. 113, figs. 1053, 1054, 1777; Gmelin, Syst. Nat., Tome I, pars. VI, p. 3225, No. 2, 1790 ; Tapparone Canefri, Muricidi del Mar Rosso, Annali del Mruseo Civico di Storia Naturale di Genova, Tol. YII, p. 572, 1S75; Tapparone-Canefri; Molluschi raccolti nelle Isole Molucche, Annali del Museo Civieo di Storia Naturale di Genora, Vol. IX, p. 294, 1876, 1877; Sowerby, Thes. Conch., Vol. IV, p. 2, Murex, pl. 1, fig. 3, 1879; Dunker, Index Molluscorum Maris Japonici, p. 3, 1882; Poirier, Revision des Murex du Muséum, Nouvelles Archives, Du MIuséum D'Histoire Naturelle, Deuxième Série, Tome V, p. 32, No. 4, $15 S 2$; Reeve, Conch. Ieon., Vol. III, pl. 20, fig. 82, 1815; Kuster, Conch. Cab., 2nd edit., p. 25, pl. 9, figs. 4, 5. II. and A. Adams, Genera of Recent IIollusca, Vol. I, Part III, p. 72, 1853, 1858; Hanley, Ipsa Limnæi Conchylia, pp. 279, 519, 1855; A. Adans, Proc. Zool. Soc., London, p. 370, 1852.

Murex crassispina, Lamarek, Anim. sans Vert., Vol. VIT, p. 157, 1822; edit. Deshajes, Vol. IX, p. 564, 1813.

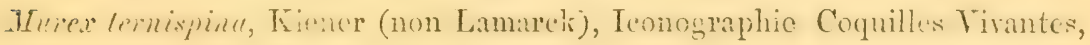
p. 6 , pl. 8, fig. 1; pl. 9, fig. 1, 1810.

Murex crassispina, Taillant, Journ. de Coneh., Vol. XIII, p. 101, No. 4, 1865 ; Fischer, Journ. de Conch., Tol. XIII, p. 241, 18:5; Issel, Malacologia del 3rar Rosso, p. 137, No. 300, 1869 ; Fischer, Jouru. de Conch., Vol. XVili, p. 163, No. 6, 1870.

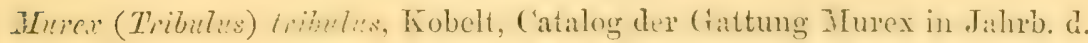
deutsch. malak. Gesellseh., Tol. IV, p. 145, No. 2, 187T; Tryon, Manual of Conchology, Vol. II, p. 77, pl. 9, fig. 107, 1880. 
Murex crassispina, E. v. Martens in Möbius, Beiträge zur Meeresfauna der insel Mauritius und der Seychellen, p. 231, 1880.

Habitat.-Nicol Bay and North West Cape, Western Australia. (J. Brazier.)

Murex tenuispina, Lamarck.

Murex Tribulus duplicatus, Chemnitz, Conch. Cab., Vol. XI, p. 103, pl. 189, fig. 1821, pl. 190, fig. 1822, 1795.

MLurex tenuispina, Lamarck, Anim. saus Tert., Vol. VII, p., 158 (edit. Deshayes), Vol. IX, p. 566. Deshayes, Encycl. Méthod., Vers. Vol. III, p. $\$ 96$, No. 4. Kiener, Iconographie Coquilles Tivantes, p. 5, pl. 6, fig. 1, pl. 7, fig. 1, 1812 ; Reere, Conch. Icon., Vol. III, pl. 21, fig. 85, 1S15; Kuster, Conch. Cab., 2nd edition, p. 27, pl. 11, fig. 3, pl. 20, fig. 3. A. Adams, Proc. Zool. Soc., London, p. 370, 1862 ; Brazier, "Chevert" Expedition Shells, Proc. Linn. Society, New South Wales, Vol. I, p. 169, 1875, 1877 ; Sowerby, Thes. Conch., Vol. IV, p. 2, pl. 1, Murex, fig. 7, 1879; Poirier, Revision de Murex du Muséum, Nourelles Archires du Muséum D'Histoire Naturelle, Deuxième Série, Tome V, p. 65,1882 ; E. A. Smith, Voyage of H.M.S. "Alert," Collections, p. 42,1881 .

Aranea trircmis, Perry, Conchology, pl. 45, fig. 3, 1811.

Murex tenuispinosus, Sowerby, Genera Recent and Fossil Shells, Tol. II, pl. 225.

IIurex duplicalus, H. and A. Adams, Genera of Recent Mollusea, Vol I, Part III, p. 71, 1853, $18 \div 8$.

Mnurex (Tribulus) tenuispina, Kobelt, Catalog der Gattung Murex in Jahrb. d. deutsch. malak. Gesellsch., Vol. IV, p. 145, No. 3, 1877.

Murex (Tribulus) tenuispina, Tryon, Manual of Conchology, Tol. II, p. 7S, pl. 50, fig. 113, 1850; Watson, Report on the Gasteropoda, Voyage of H.ML.S. "Challenger," Zoology, Vol. XV, p. 148, 1886.

IIurex (Acupurpura) tenuispina, Bayle, 1880, in Fischer Manuel de Con. chyliologie, Fascicule. VII, p. 641, 1881.

Habitat.-Darnley Island, Torres Straits, 28 to 30 fathoms, sandy botton, brought up on the tangles. (J. Brazicr.) Torres Straits, 7 to 10 fathoms. (Dr. R. W. Coppinger.) (A.M.)

Murex ternispina, Lamarck.

Murex ternispina, Lamarck, Anim. sans Vert., Vol. VII, p. 15 S (edit. Deshayes), Vol. IX, p. $56 \%$. Sowerby, Conchological Illustrations, Mures, species 2, fig. 110, 1810 ; Recre, Conch. Icon., Vol. III, pl. 1s, 
fig. 73, pl. 19, fig. 76, 1815 ; Kuster, Conch. Cab., 2nd edition, p. 57 (pl. 22, figs. 3, 4, do not represent this species). Lischke, Japanische Mecres-Conchylien, Tol. I, p. 42, 1s61-69; Issel, Malac. Mar Rosso, p. 137, No. $299,1869$.

MLurex (Tribulus) ternispina, Kobalt, Catalog der Cattung ALurex in Jahrb. d. deutsch. malak. Gesellsch., p. 145, No. 4, 1877.

MLurex ternispina, Sowerby, Thes. Conch., Vol. IV, p. 4, No. 11, Murex pl. 1. fig. 1, 1879; Poirier, Revision des Murex du Muséum, Nouvelles Archives du Muséum D’Histoire Naturelle, Deuxième Série, Tome V, p. 30, No. 1, 1882.

Mnuex (Tributus) ternispind, Tryon, Mantal of Conchnlogy, Vol. II, p. TS, pl. 9, fig. 110,1880 ; Watson, Report on the Gasteropoda, Toyage of H.M.S. "Challenger," Zoology, Vol. XV, p. 149, 1886.

Ilabitat.-Darnley Island, Torres Straits, 20 to 30 fathoms, sandy bottom, brought up on the tangles with MLurex tenuispina, Lam. (J. Brazier, "Cherert" Expedition.)

This species was inadvertently omittel from my iist of Muricide collected during the "Chevert" Expedition, Procesling $\$$ of the Linnean sucicts of New Sonth Wales, Vol. I, pp. 169, 172.

\section{Murex aduncospinosus, Beck.}

Murex aduncospinosus, Beck, M.S.

Murex temispina, Tar. Sowerby, Conchologicai Illustrations, fig. CS, 1810.

Murex aduncospinosus, Reeve, Conch. Icon., Vol. III, pl. 23, fig. 93, 1545;

Beck; A. Adlams, Proc. Zool. Soc, London, p. 370, 1862; Beck; H. and A. Adams, Genera of Recent Mollusea, Tol. I, Part III, p. 71, 1553, 1878 ; Beck; Schmeltz, jr., Muscum Godeffroy Catalog, V. p. 130, No. 6,$470 ; 1874$.

Murex (Tribulus) aduncospinosus, Beek; Kobelt, Catalog der Gattung Murex in Jahrb. d. deutsch. malak. Geselisch., Vol. IV, p. 145, No. 7, 1877.

MIurex tribuius, Sowerby, Thes. Conch., Vol. IV: p. 2, Murex, pl.1, fig. 4, 1879. Murex (Tributus) ternispina, Trgon, Mamual of Concholory, Tol. II, p. is, pl. 10, fir. 11., 1880 ; Watson, Report on the (xasteropoda, Voyage of H.MI S. "Challenger," Zoology, Vol. XV, p. 146, No. 1, 18s6.

Murex atuncospinosus, Reere (Beek); Dunker, Index MLolluscorum Mirris Japonici, p. 3, 1882 ; Poirier, Revisi sn des Murex du Muséum, Yourelles Archives du Muséum D'Ilistoire Naturelle, Deuxième Série, Tome T, p. 32, No. 4, 1882.

Habitat.-Nicol Bay, North West Coast of Australia. (IV. H. Hargraves.) Torres Straits. (Captain C. Edwards.) (A.M.) 
Murex rarispina, Lamarck.

Mairtini, Conch. Cab., Vol. III, pl. 13, fig. 1055, 1777.

MTurex rarispina, Lamarck; Anim. saus Vert., Vol. TII, p. 158, No. 5, 1 s22 ; 2nd edition, Tol. IX, p. 567. Kiener, Iconographie Coquilles Tirantes, p. 17, pl. 11, fig. 1, 1812 ; Reeve, Conch. Icon., pl. 21, fig. 86, 1815 ; H. and A. Adams, Genera of Recent Mollusca, Vol. I, Part III, p. 71, 1853.

ILrex formosus, Sowerby, Conchological Illustrations, species 16, fig. 112 ; Proc. Zool. Soc., London, p. 139, 1840. Thes. Conch., Tol. IV, 1. 6, Murex, pl. 1, fig. 6, 1879.

IIurex (Tribulus) ravispina, Kobelt, Jahrb. d. đeutsch. malak. Gescllsch, Vol. IV, p. 145, No. 9, 1877 ; Tryon, Manual of Conchology, Vol. II, p. 79 , pl. 10, fig. $115,1880$.

Murex rarispina, Poirier, Revision des Murex clu Muséum, Touvelles Archives du Muséum D’Historie Naturelle, Deuxième Série, Tome V, p. 37, No. 17, 1852 ; Hedley, Proceedings Royal Society, Qucensland, Vol. XI, Part V, p. 240, 1889.

Habitat.-Port Darwin, Northern Territory. (TT. T. Bednall, 1575.) Cambridge Gulf, Tasman Lane, North Australia. (M. Sarille-Kent.)

Murex Martinianus, Reere.

Martini, Conch. Cab., Vol. III, pl. 113, fig. 1156, 1777.

BLurex ravispina, Sowerby (non Lamarck), Conchological Illustrations, species 4 , fig. 52, 1810.

IIurcx Martinianus, Reeve, Proc. Zool. Soc., London, p. SS, 1S45. Reeve, Conch. Icon., Vol. III, pl. 18, fig. 72, 1815. Kuster, Conch. Cab., 2nd edition, p. 59, pl. 9, fig. 3, pl. 22, fig. 7,8 .

ILurex trapa, Bolten; H. and A. Adams, Genera of Recent IIollusca, Tol. I, Part III, p. 72, 1853, 1858. Bolten; Schmeltz, jr., MLuscum Godefiroy, Catalogue V, p. 130, No. 3,296, 1874.

MIurex (Tribulus) Martinianus, Kobelt, Catalog der Gattung IIurcx in Jahrb. d. deutsch. malak. Gesellsch., Vol. IV, p. 146, No. 12, 1877.

Murex rarispina, Sowerby (non Lamarek), Thes. Conch., Vol. IT, Murex, pl. 1, fig. 2, 1879.

Murex MLartinianus, Tryon, MLanual of Conchology, Vol. II, p. 7S, pl. 11, fig. 118, 18s0. Dunker, Index Molluscorum Maris Japonici, p. 3, 1882. 
Murex ILartianus, Poirier, Revision des Murex du MLuséum, Nourciles Archives du MIuséum D'Historie Naturelle, Deuxième Série, Tome $T$, p. 34, No. 10, 1882.

Muvex trapa, Bolten; Möreh, Foldi Cat., p. 98.

IIubitut.-Port Denison, North Qucensland. (Madame 1. Listrich.)

\section{Murex Mracgillivrayi, Dohrn.}

ILurex MIacgilliviraji, Dohrn, Proc. Zool. Soc., London, ]. 203, XTo. 1, 1S6:2.

BLuev brevispina, Brazier (non Lamarek), "Chevert" Expedition Shells, Proc. Linn. Soc., New South Wales, Vol. I, p. 169, No. 2, 1875, 1877.

ALurex ILuegilliwayi, Sowerby, Thes. Conch., Tol. 1V, p. 3, pl. 17, fig: 162, (wrongly coloured), 1879.

Inurex (Tributus) scolopax, Tryon, Manual of Conchologs, Tol. II, p. 77, pl. 24, fig. 208, 1880, copied from Sowerby.

ILurex Iracgillivrayi, Poirier, Revision des Murex du Muséum, Nourelles Archires du Mnséum D'Histoire Naturelle, Desième Śérie, Tome V, p. 35, No. 12, 1882 ; E. A. Smith, Voyage of H.Mr.S. "Alert," Collections, p. 44, pl. 5, fig. C, 1884 .

ILuvev (Tribulus) Macyilliverayi, Watson, Report on the Gasteropoda, Voyage of H.M.S. "Challenger," Zoology, Vol. XV, p. 147, 1886.

Habitat.-Lizard Island, North Quecusland. (John Macgillivray.) Princess Charlotte Bay, 1. fathoms sandy mud bottom; Cape Grenville, North Queensland, 20 to 30 fathoms; Cleveland Bay. (J. Brazier, "Chevert" Expedition.) Cape Tork, 6 to 11 fathoms; Port Curtis (1.M.) 10 to 11 fathoms; Port Darwin, 8 to 12 fathoms; Prince of Wales Chaunel, Torres straits, 7 to 9 fathoms. (Dr. Coppinger.) Cape Tork, off Albany Island, North Queensland, 3 to 12 fathoms (H.M.s. " ("hallenger"); Port Darwin, Northern Territory. (T. WV. Bednall, 1875.) (А.M.)

\section{Murex acanthodes, Watson.}

Murex (Tributus) acanthocles, Watson, Prelim. Report, Part XV, Journal Linnean Society, Zool., London, Vol. XVI, p. 5s9, Report on the Gasteropoda, Toyage of II.ML.S. "Challenger," Zoology, Vol. XT, p. 151, pl. 10, fig. 1, 1886.

Habitut.-Cape York, off . 11 any I land, North Australia, 3 to 12 fathoms. (H.M.S. " Challenger.) 
Murex Coppingeri, E. A. Smith.

Anrex Coppingeri, E. A. Smith, Voyage of II.M.S. "Alert," Collections, p. 42 , pl. 5, fig. $\AA, 1851$.

II abitat.-Dundas Straits, Melville Island, North Coast of Australia, 17 fathoms. (Dr. Coppinger.) Port Darwin Beach. (W. T. Bernall, 1875.)

\section{Murex acanthostephes, Watson.}

Murex (Tributus) acanthostephes, Watson, Prelim. Report, Part XV, Journal Linnean Society, Zool., London, Tol. XVI, p. 593. Watson, Report on the Gasteropoda, Voyage of H.M.S. "Challenger," Zoology, Tol. XI, p. 149, p]. 10, fig. 2, 1856.

Murex acanthostephes, E. A. Smith, Voyage of II.M.S. "Alert," Collections, p. 43, pl. 5, fig. B, 1884 .

Mabitat.-Arafura Sca, North West Cost of Australia, 32 to 36 fiatlions, bottom mud, sand, and shells. (Dr. R. WT. Coppinger.) West of Cape Tork, off South West point of New Gninea, 28 fathoms. (II.M.S. "Challenger.")

\section{Murex eximius, Brazier.}

Murex eximius, Brazier, "Cherert" Expedition Shells, Proc. Limn. Soc., New South Wales, Vol. I, p. 170, No.3, 1875, 1877. T'ryon, Manual of Conchology, Vol. IT, p. 80, 18s0).

Habitat.-Darnley Island, Torres Straits, 30 fathous, brought up on the tangles, bottom sand. (J. Brazier.)

IIUSTELLUM, Klein, 1753 ; BRONTES, Montfort, 1810 ; HAUSTELLARIA, Swainson, 1840.

Murex haustellum, Linné.

Murex haustellum, Linné, Syst. Tat., edition XII, p. 1213, No. 518, 1766. Gmelin, Syst. Nat., Vol. I, Part VI, p. 3524, No. 1, 1790.

IIaustellum fimbriatenodosum, Martini, Conch. C'ab., Vol. III, p. 376, pl. 11. fig. 1066, 1777.

Aranea denudata, Perry, Conchology, pl. 45, fig. 1, 1811.

Haustellum love, Schumacher, Nouv. Syst., p. $213,1817$.

Iurex haustellum, Lamarck, Anim. sans Vert., Vol. III, p. 159, No. S, 1S⒉ ;

2nd edition, Vol. IX, p. 568. Kiener, Iconographic Coquilles Tivantes, j. 10, pl. 13, fig. 1, 1812 ; Reeve, Conch. Icon., Vol. III, pl. 23, fig. 95, 1845; Kuster, C'onch. Cab., 2nd edition, p. 35, pl. 14, fig. 3. Hanley, Ipsa Linnæi Conchylia, p. 279, 1855. 
Murex (Haustellum) haustellum, II. and A. Adams, Genera of Recent Mollusca, Vol. I, Part III, p. 72, 1853, 1858; Schmeitz, jr., MIuscum Godeffroy, Catalog V, p. 130, No. 3297, 1874.

MIurex (Hustellum) heaustcllum, Tapparone-C'anefri, MLuricidi del Mar' Rosso, Annali del Museo Civico di Storia Naturale di Genova, Tol. VII, p. 574, 1875; Tapparone-Canefri, La Faune Malacologique de l'ile Maurice, Annales de la Société Malacologique de Belgique, Tome XV (Deuxième Série, Tome V), p. 10, 1880 ; Dunker, Index Míolluscorum Maris Japonici, p. 4, 1882; Poirier, Revision des Murex du Muséum, Nouvelles Archires du Muséum D'Histoire Naturelle, Deuxième Série, Tome V, p. 42, No. 42, 1882.

Muicer husteltum, Kobelt, Die Murieiden des rothen Mecres, Jahrb. d. deutsch. malak. Gesellsch., Vol. III, p. 10), No. 6, 1876; Kobelt, ('attalorr der Gattung Murex in Jahrb. d. dentsch. malak. Gesellsch., Vol. IV, p. 148, No. 36, 1877 ; Sowerby, Thes. Conch., Vol. IV, p. 5, Murex, pl. 2, fig. 17, 1879; Tryon, Manual of Conchology, Vol. IL, p. 83, pl. 13, fig. 137, 1850 ; E. r. MIartens, in Mobius, Beitrïge zur Meeresfauma der Insel Mauritius und der Scychellen, p. 231, 1880.

ILubitut.-Nicol Bay and Dampier's Irehipelago, North-West Iustralia. (J. F. Bailey.)

CIIICORETS, Montfort, 1810 ; CIICIIORETS, Tapparone Cimefr', 1874; CICHOREUS, E. v. Martens, 1880.

Murex ramosus, Linné.

BIurex ramosus, Linn., Syst. Nat., Ed. XII, p. 1215, 1766.

Purpurce eques firisicus, Martini, Conch. Cab., Tol. III, p. :308, pl. 102, fig. 980, pl. 103, fig. 981, 1777.

Purpura incarnata, Bolten, Museum Boltenianum, 1st edition, p. 142.

Murex iufalus, Lamarck, Inim. sans Vert., Tol. VII, p. 160, 1522; edit.

Deshayes, Vol. IX, p. 570, 1513; Kuster, Conch. Cab., 2nd edition, p. 5,

pl. $\Lambda$; pl. 1, fig. 1; pl. 2, fiø. 2. Kiener, Iconographio Coquilles

Vivantes, p. 21, pl. 1, 1S1:2; Kobelt, Die Murieiden des rothen MLeeres, Jahrb. d. deutsch. malak. Gesellsch., Vol. III, p. 40, No. 7, 1876.

Alurex ramosus, Reeve, Conch. Icon., Vol. III, pl. 1, fig. 3, 1815.

ALurex (Chicorens) fiontosus, II. and A. Adams, Genera of Recent MLolluscia, Vol. I, Part III, p. 72, 1853, 1858.

Mlurez (Chicoreus) iramosus, II. and A. Alams, Genera of lecent MFollusca. Vol. I, part III, p. 72, 1853, 1858; Brazier, "Cherert" Expedition Shells, Proe. Linn. Soc., New South Wales, Vol. I, p. 170, No. 4, 1575, 1877. 
MIurex (Chichoreus) incarnatus, Tapparone-Canefri, Muricidi del Mar Rosso, Annali del Museo Civico di Storia Naturale di Genora, Vol. TII, p. 575,1875 .

Inurex (Chicoreus) inflatus Kobelt, Catalog der Gattung Murex in Jahrb. d. deutsch. malak. Gesellsch., Vol. IV, p. 148, 1877.

IFurex ramosus, Sowerby, Thes. Conch., Vol. IV, p. 11, pl. 8, Murex, fig. 69, 1879.

Murex (Cichoreus) inflatus, E. v. Martens in Möbius Beiträge zur Mceresfauna der Insel Mauritius und der Seychellen, p. 231, 1880.

IIurex (Chicoreus) ramosus, Tryon, Manual of Conchology, Vol. II, p. 95, pl. 1, figs. 1, 2, 1880 ; Poirier, Revision des Murex du Muséum, Nouvelles Archives du Mruséun D'Histoire Naturelle, Deuxième Série, Tome V, p. 50, No. 52, 1882 .

ILure.x (Chichoreus) incarnatus, Tapparone-Canefri, La Faune Malacologique de l'ile Maurice, Mémoires de la Société Malacologique de Belgique, Tome XV (Deuxième Série, Tome V), p. 13, 1880.

Murex frondosus, Mörch, Yoldi Catalogue, p. 97.

ILwex incamatus, Mörch, Yoldi Catalogue, p. 97.

Habitat.-Darnley Island, Torres Straits, found on the recfs. (J. Brazier.) Nicol Bay, Western Australia. (J. F. Bailey.)

\section{Murex monodon, Sowerby.}

Martini, Conch. Cab., Vol. III, p. 314, pl. 105, figs. 987, 988, 1777.

Anurex Monodon, Sowerby, Tankerrille Catalogue, Appendix, p. 19, No. 1703, 1825 ; Lamarek, Anim. s. Vert. (edit. Deshayes), Vol. IX, p. 604, 1843; Reeve, Conch. Icon., Vol. III, pl. 5, fig. 21, 1845. Kuster, Conch. Cab., 2nd edition, p. 39, pl. 16, figs. 1, 2. Sowerby, Thes. Conch., Vol. IV, p. 10, pl. 8, Murex, figs. 55, 56, 1879.

IIurex aranea, Kiener, Iconographie Coquilles Virantes, p. 31, pl. 36, fig. 1, 1812 .

Ilurex (Chicoirens) monodon, Kobelt, Catalog der Gattung Mrurex in Jahrb. d. deutsch, malak. Gresellsch., Vol. IV, p. 151, 1877.

ILurex (Chicoreus) monodon, Tryon, Manual of Conchology, Tol. II, p. 9:2, p]. 16, fig. 158, 1880; Poirier, Revision des Murex du Muséum, Nourelles Archives du Muséum D'IIistorie Naturelle, Deuxieme Séric, Tome V, p. 37, No. 42, 1882.

IIurex monodon, E. A. Smith, Voyage of II.MI.S. "Alert," Collections, p. 46, No. 18, 1881. 
IFurex (Euphyllon) monoton, Jousseaume, 1850, in Fischer, Manuel de Conchyliologie, Fascicule VII, p. 641, 1881.

ILuvex (Chicoreus) monodon, Brazier, Proc. Limn. Soe., New South Trilcs, Vol. IX, p. 793, 188t, 1885; Watson, Report on the Gasteropoda, Voyage of H.M.S. "Challenger," Zoolog 5, Tol. XV, p. 154, 1886.

Ir tritat.-Dupuch Island, Western Australia. (Lientenant Dring, R.X.) Cossack, Dampier's Laud, Torth West Coast of Australia. (J. F. Bailey.) Albany Island, North Queensland, 3, 8 fathoms. (Dr. Coppinger.) Tear Cape York, 6 fathoms, coral mud." (H.M.S. "Challenger.") Nicol Bay, North-West Australia. (A.M.)

Dupuch Island is not in Torres Straits, as quoted by Reeve and other authors.

Murex cervicornis, Lamarck.

ILurex cervicomis, Lamarek, Anim. sans Vert., Vol. VII, p. 163, No. 19, 1822 (edit. Deshayes), Vol. IX, p. 575, No. 19, 1813 ; Sowerby, Genera Recent and Fossil Shells, Vol. II, Murex, pl. 22t, fig. Ł. Kiener, Iconographie Coquilles Vivantes, p. 32, pl.'20, fig. 2, 1842; Reeve, Conch. Icon., Vol. III, pl. 16, fig. 66, 1815; Kuster, Conch. Cab., 2nd edition, p. 87 , pl. 31 , figs. 5,6 .

ILurex (Chicoireus) cervicornis, II. and A. Adams, Gienera of Recent MIolluseal, Vol. I, Part III, p. 72, 1853, 1858; Kobelt, Catalog der Gattung IIurex in Jahrb. d. deutsch. malak. Gesellsch., Vol. IV, p. 152, No. 69, 1877; Brazier, "Cherert" Expedition Shells, Proc. Linn. Soc., New South Wales, Vol. I, p. 171, No.8, 1875, 1877; Tryon, Manual of Conchology, Vol. II, p. 92, pl. 15, fig. 155, 1880; Poirier, Revision des Murex du Muséum, Nouvelles Archives du Muséum D'Histoire Naturelle, Deuxième Série, Tome V, p. 46, No.4t, 1882 ; Watson, Report on the Gasteropoda, Voyage of H.M.S. "Challenger," Zoology, Vol. IV, p. 151, 1886.

Murex cevicornis, Sowerby, Thes. Conch., Tol. 1Y, p. 11, pl. 3, MLurex, fig. 30, 1879 ; E. A. Smith, Voyage of H.M.S. "Alert," Collections, p. 45, No. 16, 1884; Hedley, Proc. Royal Society, Queensland, Vol. VI, Part $V$, p. 240, 1889.

Habitat.-New Holland. (Lamarek.) Darnley Island, Torres Straits, 20, 30 fathoms, sandy mud bottom brought up in the dredge and on the tangles. (J. Brazier, 1S75.) Thursday Istand, in fithoms; Prinee of li inles Channel; Torres Straits; Clairmont Island, 11 fathoms; lort Darwin, Northem Territory, 8, 12 fathoms. (Dr. R. W. Coppinger.) Nicol Bay, Forth West Coast of Australiat, found on the beatehes after gilles: Mllany Passage, Cape Fork, North (Eucensland; oft Twofohl Bay. New South 
Wales, 150 fathoms. (J. Brazier.) Off Sydney Ireads, 80, 90 fathoms sandy bottom. (Henry Prince and T. Haylock.) Near Cape York, 6 fathoms, coral mud; West of Cape Tork, off South West point of Papua, 2S fathoms, green mud. (H.M.S. "Challenger.") Port Darwin, Northern Territory. (IV. Saville-Kent.) East Coast of Australia. (British Museum.)

\section{Murex recticornis, Martens,}

Murex recticomis, E. v. Martens; Kobelt in Jahrb. d. deutsch. malak: Geselisch., Vol. VII, p. 81, pl. 3, fig. 3, 1880.

Habitat.-East Coast of Australia, $26^{\circ} 5$ S. Lat. in 76 fathoms, obtained by the Expedition of the German War-ship "Gazelle."

The above locality is off the Queensland Coast, near WV ide Bay; this species is nearly allied to Murox cervicornis, Lam.

IMurex axicornis, Lamarek.

Martini, Conch., Cab., Vol. III, pl. 105, fig. 989, 1777.

Murex axicomis, Lamarck, Anim. sans Tert., Vol. TII, p. 163, No. 18, 1822 (2nd edition Deshayes), Tol. IX, p. 574, 1813; Kiener, Iconographie Coquilles Vivantes, p. 31, pl. 42, fig. 2, 1812; Kuster, Conch. Cab., 2nd edition, p. 4.0, pl. 16, fig. 3 ; pl. 21, fig. 3. Reeve, Conch, Icon., Vol. III, pl. 15, fig 37 ; pl. 10, fig. 37, Variety, 1845.

Murex axicomis, Tar., Somerby, Conchological Illustrations, species 30, fig. 66,1840 .

Murex (Chicoreus) axicomis, II. and A. Adams, Genera of Recent Mollusca, Vol. I, Part III, p. 72, 1853, 1858; Brazier, Proc. Linn. Soc., New South Wales, Vol. I, p. 171, No. 7, 1875, 1877; Kobelt, Catalog der Gattung Murex in Jahrb. d. deutseh. malak. Gesellsch, Vol. IT, p. 152, No. $68,1877$.

Murex axicomis, Sowerby, Thes. Conch., Tol. IV, p. 11, pl. 3, Murex, fig. 31,

1879. E. A. Smith, Voyage of H.M.S. "Alert," Collections, p. 44, No. 15, 1881 .

Murex (Chicoieus) axicomis, Tryon, Manual of Conchology, Tol. IT, p. 92, pl. 16, fig. 161, 1880; Poirier, Revision des Murex du Muséum, Nourelles Archives du Muséum D'Histoire Naturelle, Deuxième Série, Tome $\mathrm{T}, \mathrm{p} .46, \mathrm{No} .43,1882$.

ILatitat.-Palu Is]and, North East Coast of Australia, now called North (oncensland, \& fathoms, muddy bottom. (J. Brazier.) Prince of Wales Chanmel, 5, 7 fathoms, and Thursday Island, Torres Straits, 1 fathoms. (Dr. K. W. Coppinger.) (A.M.) 
Murex Sauliæ, Sowerby.

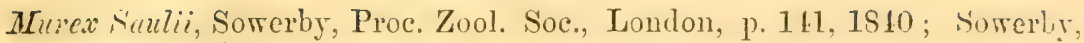
Conchological Illustrations, species 34 , fig. 77, 1810; Deshayes in Lamarek, Anim. sans. Vert., Vol, IX, p. 607, No. 76, 1813.

Irurex palmarose, Var., Kiener, Iconographie Coquilles Vivantes, p. 29, 1s 12.. IIurex Saula, Reeve, Conch. Iron., Vol. III, pl. 8, fig. 31, $18 \pm 5$.

ALurex (Chicoreus) Saulice, II. and A. Adams, Genera of Recent MLollusia. Vol. I, Part III, 1853, 1S58; Kobelt, Catalog der Gattung Murex in Jahrb. d. deutsch. malak. Gesellsch., Vol. IV, p. 149, No. 41, 1877.

Mruiex Stutii, Liénard, Catalogue de lia Fitune Malacologique de liile Maurice, p. 2, No. 14, 1877.

ALurex Saulce, Sowerby, Thes. Conch., Vol. IV, p. 13, pl. 4, Murex, fig. 41; pl. 22, fig. 212, 1879.

Murex (Chichoreus) Śaulice, Tapparone-Cancfri, La Faune Malacologique de

l'ile Maurice, Mémoires de la Société Malacólogique de Belgique,

Tome XV (Deuxième Série, Tome V), p. 12, 1880.

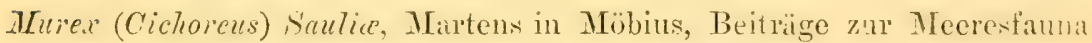
der Insel Mauritius und der Seychellen, p. 232, 1880.

ILurex (Chicoieus) maurus, Var., Tryon, Manual of Conchology, Tol. II, p. s?, pl. 1t, fig. 142, 1880.

Inurex (Chicoreus) sunlle, Poirier, Rerision des Murex du Musíum, Tourelles Arehires du MIuséum D'IIistoire Naturelle, Denxième S'́rie, Tome V, p. 54, No, 61, 1882 .

IEabitat.-Torres Straits, North Coast of Australia. (W. II. Margraves.) Tlaming Head, North West Cape, Western Australia. (J. Brazier.) (A.M.)

\section{Murex torrefactus, Sowerby.}

Murex torrefuctus, Sowerby, Pror. Zool. Soc., London, p. 111, 1817) Sowerhy, Conchological Illustrations, species 35, fig. 120, 1810; Reere, Conch. Icon., Vol. III, pl. 10, fig. 41, 1815 ; Sowerby, Thes. Conch., Vol. IV, p. 15, pl. 5, Murex, fig. 17, 1879.

Inurex microplygllus, Kiener (nou Lamitrek), Ieonographie (onquilles Vivantes, pl. 23, fig. 1, 1812.

Murex (Chicorens) torrefactus, Kobelt, Catalog der Gattung I[urex in Jahrb. d. deutsch. malak. Gesellsch., Vol. IV, p. 150, No. 47, 1877; Tryon, Manual of Conchology, Vol. II, p. 89, pl. 1t, fig. 145, 1880; Poirier, Revision des Murex du Muséum, Nourelles Archives du Muséum D'Histoire Naturelle, Deuxième Série, Tome V, p. 56, No. 66, 1852.

ILabitat.--Nicol Bay, North West Coast of Australia. (IV II. IIargraves) (A.MI.) 
Murex microphyllus, Lamarck.

Purpura rariegata, Martini, Conch. Cab., Vol. III, pp. 30こ, 322, pl. 106, fig. 995,1777 .

ALurex microphyllus, Lamarek, Anim. sans Tert., Vol. VII, p. 163 (edit. Deshayes), Vol. IX, p. 575. Sorverby, Conchological Illustrations, species 38, fig. 105, 1841; Reeve, Conch. Icon., Vol. III, pl. 10, fig. 40, 1815 ; Kuster, Conch. Cab., 2nd edition, p. 18, pl. 6, fig. 3.

MLurex (Chicoreus) microphyllus, H. and A. Adams, Genera of Recent Mollusea, Tol. I, Part III, p. 72, 1853, 1858; Schmeltz, jr., Museum Godeffroy Catalog V, p. 103, No. 1,057, 187t; Kobelt, Catalog der Gattung Murex in Jahrb. d. deutsch. malak. Gesellsch., Vol. IV, p. 149, 1877; Tryon, Manual of Conchology, Vol. II, p. 89, pl. 14, fig. 144, 1580; Tapparone-Canefri, La Faune Malacologique de l'ile Maurice, Mémoires de la Société Malacologique de Belgique, Tome XV (Deuxième Série, Tome V), p. 19, 1880.

Murex (Cichoreus) microphyllus, E. r. Martens in Möbius Berträge zur

Meeresfauna der insel Mauritius und der Seychellen, p. 232, 1880;

Poirier, Revision des Murex du Muséum, Nourelles Archives du Muséum

D'Histoire Naturelle, Deuxième Série, Tome V, p. 56, No. 65, 1882.

Habitat.-Torres Straits. (C. E. Beddome, Dr. J. C. Cox.)

\section{Murex maurus, Broderip.}

Mure. manrus, Broderip, Proc. Zool. Soc., London, p. 17t, 1832; Kiener, Iconographie Coquilles Virantes, p. 39 (Murex adustus, in part); Sowerby, Conchological Illustrations, species 39, figs. 12, 67, 1810 ; Reeve, Conch. Icon., Tol. III, pl. 4, fig. 16, 1845; Deshayes in Lamarck, Anim. saus Vert., Vol. IX, p. 573, note 1813 ; Sowerby, Thes. Conch,, Tol. IV, p. 15 ; Murex, pl. 5, fig. 54, 1879.

IInex (Chicoreus) maurus, Kobelt, Catalog der Gattung Murex in Jahrb. d. deutsch, malak. Gesellsch., Vol. IV, p. 150, No. 48, 1877; 'Tryon, Manual of Conchology, Tol. II, p. 89, pl. 14, fig. 139, 1880; Poirier. Rerision des Murex du Muséum, Nouvelles Arehives du Muséum D'Histoire Naturelle, Deuxième Série, Tome V, p. 59, No. 70, 1882; Watson, Report on the Gasteropoda, Toyage of II.M.S. "Challenger," Zoology, Vol. XV, p. 154, 1886.

ILabitat.-Cape York, off Albany Island, Torres Straits, 3 to 12 fathoms. (H.M.S. "Challenger.") Holborn Island, off Port Denison, Queensland, 6 fathoms, sand bottom. (J. Brazier.) 
Murex Banksi, Sowerby.

MIurex Bankisi, Sowerby, Proc. Zool. Soc., London, p. 111, 18t0; Conchological Illustrations, species 31, fig. 82, 1810; Kiener, Iconograplie Coquilles Virantes, p. 36, pl. 21, fig. 1; Reere, Conch. Icon., Vol. III, pl. 10, fig. 38, 1845; Kuster, Conch. Cab., 2nd edition, p. 120, pl. 36, fig. 6 ; Sowerby, Thes. Conch., Vol. IV, p. 17 ; Mures, pl. 6, fig. 59.

Murex (Chichoreus) Banksii, Tapparone-Canefri, Muricidi Del Mar Rosso, Annali del Museo Civico di Storia Naturale di Genova, Vol. VII, p. $580,1875$.

Murex (Chicoieus) Banksii, Kobelt, Catalog der Gattung Murex in Jahrb. d. deutsch. malak. Gesellsch, Tol. IV, p. 150, No. 54, 1877 ; Tryon, Manual of Conchology, Vol. II, p. 89, pl. 14, fig. 141, 1880.

Habitat.-Nicol Bay, North West Australia. (A.M.) (Mr. WV. II. Hargraves.)

\section{Murex adustus, Iamarck.}

Puppure Brandaris niger, Martini, Conch. Cab., Vol. III, 1). 317, pl. 105, figs. 990, 991, 1777.

Pupura scabra, Martyn, Lniversal Conchologist, Tol. II, fig. 113, 17 S9.

Iurex adustus, Lamarck, Anim. sans Vert., Vol. TII, p. 162 (edition Deshayes), Tol. IX, p: 573.

Triplex flavicunda, Perry, Conchology, pl. 6, fig. 2, 1811.

Triplex rubicunda, Perry, Conchology, pl. 6, fig. 4, 1811.

Murex adustus, Blainville, Manuel de Malacologie et Conchyliologie, Tol.

I, p. 401; Tol. II, p1. 19, fig. 1, 15:5; Kiener, Jconographie C'oquilles Virantes, p. 35, pl. 33, fig. 1, 1812; Menke, Molluscorum Norae Hollandiae, p. 24, No. 110, 1843 ; Reere, Conch. Icon., Vol. III, pl. S, fig. 29, 1815; Kuster, Conch. Cab., 2nd edition, p. 31, pl. 13, fig. 5; Eydoux et Souleyet, Toyage de la "Bonite" Zoologie, Tol. II, 1). 619, Atlas pl. 44, figs. 3, 5, 1852 ; A. Adams, Proc. 'Zool., Soc., London, p. 371,1862 .

Mfurex despectus, A Adams, Proc. Zool. Soc., London, p. 72, 1853.

Murex (Chicoreus) adustus, E. $\Lambda$. Smith, Ammals Mag. Nat Il istor, I series, Vol. XV, p. t19, No. 20, 1875; Brazier, "Chevert" Expentition Shells, Proc. Limn. Soc., New South Wales, Vol. T, p. 170, No.5, 1876 ; Lobelt, Catalog der Gattung Murex in Jilhrb. d. dentreh. malak. Gesellseh, Tol. IV, p. 149, 1s77; Tryon, Manual of Conchologs, Tol. 1I, p. 90, pl. 15, fig. 149, pl. 24, fig. 211, 1880 ; E. v. Martens in Mübius, Beitrïge zur Mecresfauma der Insel Mauritius und der Seychellen, p. 232, 1850; 
Tapparone-Canefri, La Faune Malacologique de l'ile Maurice, Mímoires de la Société Malacologique de Belgique, Tome XV (Deuxième Súries, Tome V), p. 11, 1880 ; Dunker, Index Molluscorum Maris Japonici, p. 4, 1832 ; Poirier, Revision des Murex du MIuséum, Souvelles Archires du Muséum D'Histoire Naturelle, Deuxième Série, Tome T, p. 59, No. 72, 1892 ; E. A. Smith, Marine Mollusea of St. Helena, Proc. Zool. Soc, London, p. 258, 1890

Murex adustus, Liénard, Catalogue de la Faune Malacologique de l'ile

Maurice, p. 2, No. 3, 1877 ; Sowerby, Thes. Conch., Vol. IV, p. 16, Murex,pl. 4, figs. 42, 43, 1879.

IIurex despectus, Sowerby, Thes. Conch., Tol. IV, p. 16, Murex, pl. 9, fi心. 85, 1879.

Habitat.-Darnley Island, Torres Straits; found on the reefs at low water, under coral and stones. (J. Brazicr.) King's Sound, North-TVest Australia. (A.M.)

Murex capucinus, Chemnitz.

Martini, Conch, Cab., Vol. III, pl. 105, fig. 993, 1777.

Murex monachus capucinus, Chemuitz, Conch. Cab., Tol XI, p. 123, pl. 192, fig. 1819, $1850,1795$.

ILirex remosus, Variety C, Dillwyn, Descriptive Catalogue of Recent Shells, Vol. II, p. 657, 1817.

Murex capucinus, Lamarek, Anim. sans Vert., Vol. VII, p. 161, 1522 (2nul edition, Deshayes). (Vol. IX, p. 576, 1813); Deshayes, Encyclopédie Méthodique Histoire Naturelle Des Vers, 'Tome III, p. 900, No. 1:2, 1832; Lamarck; Sowerby, Conchological Illustrations, p. 3, specics 40, 1810 ; Lam.; Kiener, Coquilles Tivantes, p. 42, p1. 45, fig. 2, 181.2.

Murex capucinus, Lam.; Menke, Molluscorum Novae Hollandiae, p. 24, No. 111, 1843. Chemnitz; Reeve, Conch. Icon., Vol. III, pl. 2, fig. 10, 1815. Lamarck; Kuster, Conch. Cab., 2nd edition, p. 32, pl. 13. figs. 6, 7. Chemnitz; Tapparone-Canefri, III. Contribuzioui Per Una Fauna Malacologica Delle Isole Papuane, Annali del Museo Cirico di Storia Naturale di Genora, Vol. VIII, p. 323, 1876.

Murex (Chicoreus) capucinus, Chemnitz; H. and A. Adams, Genera of Recent Mollusca, Vol. I, Part III, p. 72, 1853, 1858; Chemnitz; Sichmeltz, jr., Muséum Godeffroy Catalog V, p. 130, No 591, 1871. Lamarck; Kobelt, Jahrb. d. deutsch. malak. Gesellsch., Tol. IV, p. 15(), No. 50, 1877; Tryon, Manual of Conchology, Vol. II, pp. 99, 194, pl. 19, fig. 174, 1880; Poirier, Revision des Murex du Muséam, Nourelies Archives du Muséum D’Histoire Naturelle, Deuxième Sćrie, Tome V, p. 63, No. 85, 1882. 
Airuex (Chichoreus) capucinus, Tapparone-Canefri, Contribuzioni Per L'ni Fauna Malacologiea Delle Isole Papuane, Annali del Museo Civico di Storia Naturale di Genova, Vol. VI, p. 550, 187 .

Murex capucinus, Chemnitz; Sowerby, Thes conch., Vol. IV, p. 18, МГur. pl. 1, fig. 40, 1579; Hedley, Proc. Royal Society, Quecnsland, Tol. VI. Part V, p. 210, 1889.

Habitat.-Torres straits. (A.MI.) Port Darwin, Northern Territory. (T. W. Bednall, 1875.) Port Essington, Coburg Peninsular, Northern Territory. (E. Spalding.) Great Barrier Reef, east of Cape Sidmouth. North Quecusland; found under coral blocks at half tide (J. Brazier, "Eclipse" Expedition, December, 1S71.) ('ambridge Gulf, North-West Australia; Port Darwin, Northern Territory; Barrier Reef. (W. SarilleKent, 1888.)

\section{Murex Australiensis, A. Adams,}

Murex Australiensis, A. Adams, Proc. Zool. Soc., London, p. 72, 1853. Sowerby, Thes. Conch., Vol. IV, p. 16, Murex, pl. 6, fig. 58, 1879.

Murex (Ocinebra) Austratiensis, Kobelt, Catalog der Gattumg Murex in Jahrb. d. deutsch. malak. Gesellsch., Vol. IV, p. 218, No. 221, 1877.

Murex (Chicoreus) ridustus, Tar. Tryon, Manual of Conchology, Tol. II. P. 90, pl. 24, fig. 210, 1880.

Murev (Chicoreus) Austratiensis, Poirier, Revision des Murex du MLuséum Nourelles Archives du MLuséum D'Iistoire Naturelle, Deuxième Xérie. Tome V, p. 61, No. 75, 1882.

Habitat.-Australia (Mus. Cuming in British Musemm). Wreck Rect, Coral Sea, off the North Queensland Coast, outside the Great Barrier Reeft. (J. Brazier, 1856.) (A.M.)

This species is not found in South Australia as quotod by Mr. G. B. Sowerby in 'Thesaurus Conchyliorum, Murex, pl. 6.

\section{Murex Huttoniæ, Wright.}

Murev Finttonice, Wright, Annales de la Soeiété Malacologique de Belgique. 'Tome XIII (Deuxieme series, Tome III), p. 8.5, pl. 9, 187s; Sowerly, Thes. Conch, Vol. IV, p. 17, Murex, pl 6, fig. 57, 1879.

Murex (Chicoreus) adusius, Variety, Tryon, Manual of Concholong, Tul. IT, p. 90 , pl. 25 , fig. 217,1880

Murex (Chicoreus) IIttonice, Poirier, Revision des Murex du Muséum, Nourelles Archires du Muséum D’ Histoire Naturelle, Deuxième Súrie, Tome V, p. 60, No. 71, 1882. 
Habitat.-New Caledonia. (Wright.) Wreck Reef, Coral Sea, off the North Queensland Coast, outside the Great Barrier Reef; Chesterfield Shoals and Island, and Bampton Reef, West North West from New Caledonia. (J. Brazier, 1855, 1856.) (A.M.)

These two species, MI. Australiensis, A. Adams, and M. Huttonice, Wright, are classed by Mr. Tryon, who was only aequainted with these shells by figures and not by specimens, in his Manual as mere varieties of $M I$. adustus, Lamarck. In this he is wrong, for they are certainly quite distinct from that species, both as regards fronds, sculpture, and colour.

Murex australis, Quoy and Gaimard.

Tripler pondose, Perry, Conchology or the Natural History of Shells, pl. TI, fig. 1, 1811.

Murex australis, Quoy et Gaimard, Voyage de "l'Astrolabe," Tome II, 1". $536,1832$.

ILurex palmiferus, Sowerby, Proc. Zool. Soc., London, p. 142, 1S40; Conchological Illustrations, species 43, fig. 104, 1810; Reeve, Conch. Icon., Vol. III, pl. 4, fig. 20, 1845 .

IIurex (Chicorens) patmiferus, H. and A. Adams, Genera of Recent Mollusca, Tol. I, Part III; p. 72, 1853, 1858; Angas, Proc. Zool. Soc., London, p. 186, No. 6, 1867 ; Kobelt, Catalog der Gattung Murex in Jahrb. d. deutsch. malak. Gesellsch., Vol. IV, p. 150, No. 55, 1877; Tryon, Manual of Conchology, Vol. II, p. 90, pl. 14, fig. 146, 1850; TapparoneCanefri, La Faune Malacologique de l'ile Maurice, Annales de la Société Malacologique de Belgique, Tome.XV (Deuxiéme Sèrie, Tome T), p. 10, 1880; Watson, Report on the Gasteropoda, Voyage of H.M.s. "Challenger," Zoology, Vol. XV, p. 155, 1886.

ALurex (Chichoreus) palmiferus, Tapparone-Canefri, Muricidi Del Mar Rosso, Annali del Museo Civico di Storia Naturale di Genova, Vol. VII, p. $580,1875$.

Ilurex palmiferus, Liobelt, Die Murieiden des rothen MLeeres, Jahrb. d. deutsch malak. Gesellsch, Vol. III, p. 42, No. 14, 1876 ; Suwerby, Thes. Conch., Tol. IV, p. 18, No. 84, pl. 4, Murex, fig. 41, 1879.

MIurex (Tribulus) australis, Tryon, Manual of Conchology, Tol. II, p. 83, 1880.

Murex (Chicorens) anstralis, Poirier, Revision des Mures du Muséum, Nouvelles Archives du Muséum D'IIistoire Naturelle Deuxième Série, Tome V, p. 32, No. 4, 1882. 
Murex palmifems, Sowerby; Whitelegge, List of the Mimine and Fren Water Invertebrate Fauna of Port Jackson and Neighbourhod, Journal and Proceedings of the Royal Society of New South Wales, Tol. XXIII, p. 246, No. 208, 1889.

Habitat.-Port Western, New Holland. (Quoy et Gaimard, 1829.) Sydney. (Dutailly.) New Holland. (M. J. Verreaux, 1816.) New Caledonia. (M. Deshayes, 1879.) Red Sea. (Sowerby, 1810.) North Australia. (Reere, 1815.) Between Balls Head and Goat Island, 18 fathoms, bottom broken shells, stones, and gravel; Point Piper, Middle. TIarbour, Watson's Bay, under stones at low water spring tides; Cape Solander, South Head, and Cape Banlis, North IIead, of Botany Bay; South Head of Crook Haven River, Shoalbaven, found under stones low water springs; Montague Roads, Jervis Bay, near the rocks in 5 fathoms; Nelson Head and Bay, Port Sitephens, New South Wales, under stones low water spring tides. (.J. Brazier, 1851, 18.22.) Woolloomooloo Bay. (G. F. Angas.) Neutral Bay, Port Jackson. (T. Whitelegge.) Teppoon, Keppell Bay, Queensland. (George L. Pilcher, 1857.) Largs Bay, South Australia. (Arnold U. Henn, 1891.)

I have not seen this species any farther south of Sydney than Jervis Bar, where I dredger specimens on Oetober $31,187 t$. The specimeus said te be obtained by Quoy and Gaimard at Port Western, New Holland (now Tictoria), may have been dredged by them. I had not seen any species like it from Southern Australia, until quite recently, when two specimens mere obtained from shell debris, by my friend Mr. Arnold C. Henm, at Largs Bay, South Australia, in Norember, 1891.

I quote M. M. J. Poirier, from the Nourelles Archives du Musémm D'Histoire Naturelle, p. (6.): "This species, of Quoy and raimard, misunderstood by all authors, has nearly the same geographical range as M. commyutus, Sorrerby, with which, besides, it has many analogies. It has heen remarked on the northern coasts of Australia and in the Red Sea. It is represented by twelve individuals coming from Port Trestern (the types of Quoy and Gaimart, 1S29), from Sydney (Coll. Dutailly), flom New Holland (M. J. Verreaux, 1816), and from New Caledonia (II. Deshayes, 1574). The $M$. austialis, Quoy and $\mathrm{G}$., not figured in the atlas of the voyage of the "Astrolabe," has beren lost in forgetfulness by the various. writers who have deait with the genus Murex. Tryon alone almits it, but without recognising its affinities, and he places it anong the group of Tilbulus. An examination of the types preserred in the collection of the Museum* has shown me that this species is no other than that described hy sowerby under the name of $M$. palmifreus. This denomination buing the latest, ought then to pass into the synonomy." 
The Rev. Boog Watson, in his Report on the Gasteropoda of H.M.S. "Challenger," Zoology, Vol. XV, p. 155, appears to have followed Mr. Tryon in lumping MI. palmiferne, Sowb., = australis, Quoy and G., with three distinet and well known species, namely, Murex corrugatus, Sowb.; IIurex multifrondosus, Sowb. ; and Murex dilectus, A. Adams.

\section{Murex corrugatus, Sowerby.}

Inurex corrugutus, Sower'sy, Proc. Zool. Soc., Iondon, p. 112,1840 ; Conchological Illustrations, species 4t, fig. 72, 1810; Kiencr, Iconographie Coquilles Vivantes, p. 43, No. 32, pl. 19, fig. 2, 1812; Reere, Conch. Icon., Vol. III, pl. 13., fig. 52, 1815; Kuster, Conch., Cab., 2nd edition, p. 73, pl. 26, fig. 3. Taillant, Recherche sur la faune Malacologique de la baie de Suez, Journ. de Conch., Tol. XIII, p. 105, No. 5, 1565; Fischer, Faune Conchyliologique de Suez IIer Rouge, Journ. de Conch., Vol. XIII, p. 244, 1865.

ALurex (Chicoreus) corrugatus, II. and A. Adans, Genera of Recent Mollusen, Vol. II, Part III, p. 72, 1853, 1858.

MIurex corrugatus, Issel, Malacologia Del Mar Rosso, p. 135, No. 292, 1869.

Fischer, Sur la Faune Conchyliologique marine des baies de Suez et l'Akabah, Journ. de Conch., Vol. XVIII, p. 163, No. 5, 1870.

IIurex (Chicoreus) corrugatus, Brazier, Proc. Limn. Soc., New South Wales, Tol. I, p. 171, No. 6, 1875, 1877; Tapparone-Canefri, Muricidi dèl Mar Rosso, Annali del Museo Civico di Storia Naturale di Genora, Tol. VII, p. 579, 1875; Kobelt, Catalog der Gattung Murex in Jahrb. d. deutsch. malak. Gesellsch., Vol. IV, p. 150, No. 57, 1877; Var., Tryon, Manual of Concholog., p. 90, pl. 14, fig. 147, 1880 ; Poirier, Revision des Murex du Muséum, Nouvelles Archives du Muséum D’Histoire Naturelle, Deuxième Série, Tome V, p. 64, No. 89, 1852.

Murex corrugatus, Kobelt, Die Muriciden des rothen Meeres, Jahrb. d. deutsch. malak. Gesellsch, Vol. III, p. 42, No. 13, 1876; Sowerby, Thes. Conch., Vol. IV, p. 19, No. S9, Murex, pl. 9, fig. 72, 1879.

Habitat.-North Australia. (Reeve.) Palm Island, North East Coast of Australia, now called North Queensland; two specimens brought up on the tangles from 8 fathoms, muddy bottom. (J. Brazier.)

\section{Murex territus, Reeve.}

Murex tervitus, Reeve, Proc. Zool. Soc., London, p. 108, 1845; Reere, Conch. Icon., Vol. III, pl. 33, fig. 167, a, b, 1815. 
MIurex (Chicoreus) teritus, II. and A. Adams, Generia of Recent Mollusen, Vol. I, Part III, p. 733, 1553, 1858; Kobelt, Catalog der Gattung MLurex, in Jahrb. d. deutsch. malak. Gesellsch., Vol. IV, p. 151, 1877; Tryon, Manual of Conchology, Vol. II, p. 91, pl. 15, fig. 152, 1880; Poirier, Revision des Murex du Muséum, Nouvelles Archives du Muséum D'Histoire Naturelle, Deuxième Série, Tome V, p. 62, No. 83, 1882.

Mnerex mubilus (=territus, jun.), Sowcrby, Proc. Zool. Soc., London, p. 128, pl. 49, fig. 4, 1859 .

MLurex nubilus, Sowerby, Thes. Conch., Tol. IT, p. 7, MLurex, pl. 9, fig. 71, 1879.

Murex territus, Suwcrby, Thes. ('onch., Vol. IT, p. 19, Mures, pl. 9, fig. Ti, 1879 ; E. A. Smith, "Alert" Collections, p. 45, No. 17, 1881.

Habitat.-North Australia. (Lieutenant Ince, R.N.) Wide Bay and Port Curtis. (British Museum.) Between Home Islands and Cape Grenville, North East Coast of Australia, now North Qucenslaud, 20-30 fathoms, sandy-mud bottom, brought up on the tangles. (J. Brazier, "Cherert" Expedition.) Port Molle, 15 fathoms, and Port Curtis, 0-11 fathoms. (Dr. R. W. Coppinger.) Port Curtis. (A.M.)

This species was inadvertently omitted from my list of Muricidx collected during the "Cherert" Expedition, Procecdings of the Limnean Society of New South Wales, Tol. I, pp. 169, 172. And it is not found at Sydner, Australia, as quoted by Mr. Tryon in his Manual of Conchology, Tul. II, p. 91.

PHILLONOTUS, Swainson, 1510; MLURICANTHUS, Swainson, 1510 ; CENTRONOTUS, Swainson, 1835.*

\section{Murex Stainforthi, Reeve.}

Murex Stainforthi, Reere, Proc. Zool. Soe., p. 104, 1S12; Conch. Teon., Vol. III, pl. 17, fig. 68, 1S45; Sowerby, Thes. Conch., Vol. IV, p. 36, Murex, pl. 16, fig. 158, 1879.

ALurex (Phyllonotus) Stainforthi, II. and A. Adams, Genera of Recent 1[ollusea, Vol. II, Part III, 1). 74, 1853, 1858; Tíbelt, Catalog der Gattung MIurex in Jahrb. d. deutsch. malak. Gesellsch., Vol. IV p. 158, 1877; Tryon, Manual of Conchology, Vol. II, p. 104, pl. 26, fig. 237, 1880 ; Poirier, Rerision des Mrurex du Muséum, Nourelles Arehives du Muséum D’IIstoire Naturelle, Denxieme Ś́rie, Tome T, p. 83, No. 138, 1882.

Murex (Bussia) Stainforthi, Bayle, 1850 in Fiseher Mranuel de Conchyliologie, Fascicule, VII, p. 641, 1881 .

\footnotetext{
* This name had been previously giren to a genus of Fishes in 1801.
} 
Habitat.-Nicol Bay, North West Australia.

(IV. II. Hargraves.) Port Dar'win and Port Essington, North Australia. (T. W. Bednall and E. Spalding.) Two specimens are represented in the Museum of the Jarlin des Plantes, Paris, said to have been found in New Caledonia. (MI. J. Poirier.)

\section{Murex endivia, Lamarck.}

Martini, Conch. Cab., Vol. III, pl. 107, fig. 1008, 1777.

Murex cichoreum, Gmelin, Syst. Nat., p. 3530.

Murex endicin, Lamarck, Anim. sans Vert., Tol. TII, p. 168, 1822; edit. Deshayes; Vol. IX. p. 583. Crouch; Lamarek, Conch., pl. 18, fig. 1. Deshayes, Enc. Meth., Vers. III, p. 902. Sowerby, Conchological Illistrations, No. 92, 1811; Kiener, Iconographie Coquilles Tivantes, p. 52, pl. 35, fig. 1, 1842 ; Reeve, Conch. Icon., Vol. III, pl. 7, fig. 2ז, b, c, d, 1845; Kuster, Conch. Cab., 2nd edition, p. 42, pl. 17, fig. 7 . Sowerby, Thes. Conch., Vol. IV, p. 32, Murex, pl. 16, figs. 153, 151, 1879 ; Gatliff, List of some shells of the Marine Mollusca found on the Victorian Coast, Victorian Naturalist, Vol. IV, p. 57, No. 4, 1887.

Murex saxatitis, Wood, Index. Testac., pl. 25, fig. 8.

Murex (Phyllonotus) endivia, Kobelt, Catalog der Gattung M[urex in Jahrb.

d. deutsch. malak. Gesellsch., Vol. IV, p. 158, 1877 ; Tryon, Manual of Conchology, Vol. II, p. 102, pl. 26, fig. 227, 1880.

Habitat.-Portland Bay, Victoria, Southern Australia. (J. A. Gatliff.)

This species was sent to me for name some years ago by Mr. Gatliff, who informed me that he obtained it at the above locality. Since then, I have been informed that it was brought with other shells from the Indian Ocean. Probably, the species may be found on the west or north-west coast of Australia, but I have not seen any species like it from there.

Murex umbilicatus, Tenison-Woods.

Ilurex scalaris, A. Adams (non Brocchi), Proc. Zool. Soc., London, p. 71. 1853; Angas (non Brocchi), Marine Molluscan Fauna of South Australia, Proc. Zool. Soc., London, p. 157, No. 5, 1865.

Tiopthon umbiticatus, Tenison-Woods, Papers and Proceedings of the Royal Society of Tasmania, p. 135, 1875, 1876. Census; with brief deseriptions of the Marine Shells of Tasmania and the adjacent islands. Papers and Proceedings of the Royal Socicty of Tasmania, p. 26, $1877,1878$.

Alurex (Aluricinea) sealaris, Brazier (non Brocchi), "Chevert" Expenlition Shells, Proc. Linn. Soc., New South Wales, Vol. I, p. 172, No. 13, $1875,1877$. 
Murex (Ocinelra) scalaris, Kobelt (non Brocehi), Jahrb. d. deutsch. malal. Gesellsch., Vol. IV, 1. 248, No. 227, 1877.

Murex (Phyllonotus) Angasi, Tryon (non Crosse), Manual of Conchology, Vol. II, p. 109, 1880.

Murex (Phyllonolus) octogonus, Bednall (non Quoy and Gaimard), Trimsaletions and Proceedings and Reports of the Royal Society of South Australia, Vol. VIII, p. 61, 1851, 1885 (issued May, 1886).

Habitat.-Moreton Bay, Qucenstand. (Mrr. F. Strange.) St. Vincent's Gulf, South Australia, "dredged along with horny zoophytes and mullipores at a depth of seven or eight fathom." (Angas, Tate, Bednall). Darnley Island, Tomes Straits, 30 fathoms, sandy bottom. (J. Brazicr.) Barran Island, Bass Strait. (Mr. W. Legraml, 1573.) Giles Point, near Coubowie, St. Vincent's Gulf, oft the rocks below low water mark. (Mr. E. II. Matthews, 1S91.) Trowbridge Island, st. Tincent's Gulf, off the heach, also dredged in life from 1 to 10 fathoms ; fair specimens from IIardwicke Bay, Spencer's Gulf. (M[r. E. H. Matthews, 1892.) East Coast of Tasmania. (M[r. W. Legrand.) North ('oist of Tasmania from Port Sorell to a few miles west of the River Leven, specimens generally rery much beach worn. (Miss Mary Lodder, August, 189.2.)

\section{PTERONOTUS, Swainson, 1810.}

Murex Bednalli, Brazier.

Murex (Pteronotus) Bcthalli, Brazier, Prui. Limn. Soc., New South Walex, Vol. II, p. 6, 1877 ; Angas, Proc. Zool. Soc., Tondon, p.418, pl.45, fig. 2, 1880 ; Brazier, Proc. Linn. Soc., New South Wales, Vol. V, p. 630, 1850; Tryon, Mamual of Conchology, Tol. II, Addenda, p. 262, 1556). Murex (Cerastoma) bednalli, Poirier, Rerision des Murex du Musimm, Touvelles Arehives du IIuséum 1'Jlixtuire Vaturelle, Deuxiome sírié, Tome V, p. 98, No. 185, 1882.

Habitat.-Port Darwin, Northern Territory. (T. W. Bednall.)

\section{Murex triformis, Reere.}

Murex acanthopterus, Titr., Sowerlyg, Concholongical Illustrations, fig. .51, 1S.10.

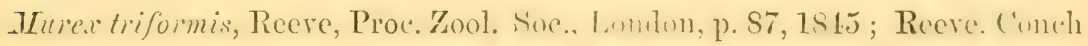
Icon., Vol. IIT, pl. 13, fig. 53, 1845; Kuster, Conch. Cab., 2nd edition, p. 85, pl. 30 , fig. 4 .

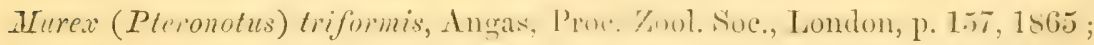
Schmeltz, jx., Museum Godeflroy Catalog V, p. 131, No. 6,291, 1874; Kobelt, Jahrb. d. deutseh malak. Gesellsch., p. 161, No. 138, 1877 ; 
Tryon, Manual of Conchology: Tol. II, p. 8.5, pl. 40, fig. 511, 1850, 1852 ; Poirier, Revision des Murex du Muséum, Nourelles Archives du Mruséum D'Histoire Naturelle, Deuxième Série, Tome T, p. 92, No. 162, 1582 ; Bednall, Transactions and Proceedings and Report of the Royal Society of South Australia, Tol. VIII, p. 63, 1885 (1836); Watson, Report on the Gasteropoda, Torage of H.M.s. "Challenger," Zoology, Vol. XV, p. 156, 1886.

ILurex triformis, Sorerby, Thes. Conch., Tol. IV, p. 21, pl. 2, Murer, fig. 92, pl. 13, fig. 126, 1879.

Habitat.-Port Lincoln, Spencer's Gulf, South Australia (A.M.), on ofster-banks, from 2 to 10 fathoms. (G. F. Angas.) Off East MIoneœur Lsland, Bass Strait, 38 fathoms, sand and shells. (II.MI.S. "Challenger.") Tasmania.

\section{Murex acanthopterus, Lamarck.}

Murex acanthoptcrus, Sehröter, Einleitung in die Conchylien-Konntniss, Vol. I, pl. 3, fig. 8, 1783; Lamarek, Anim. sans Vert., Vol. VII, p. 165 (edit. Deshayes), Vol.IX, p.577; Sowerby, Conchological Illustrations, species 59, fig. 85, 1810; Kiener, Iconographie Coquilles Vivantes, p. 105, pl. 38, fig. 2, 1842 ; Reeve, Conch. Icon., Tol. III, pl. 16, fig. 61 , 1845; Wood, Index Testaceologicus (edit. Hanley), p. 132, pl. 27, fig. 91, 1856 ; Kuster, Conch. Cab., 2nd edition, p. 98, pl. 34, fig. 2. Sowerby, Thes. Conch., Vol. IV, p. 21, pl. 10, Murex, fig. 90, pl. 13, fig. 127, 1879.

MLurex (Pteronotus) acanthopterus, Angas, Port Jackson Mollusea, Proc. Zool. Soc., London, p. 186, 1867 ; Kobelt, Jahrb. d. deutsch. malak. Gesellsch., p. 238, No, 141, 1877; Tryon, Manual of Conchology, Tol. II, p. 85, pl. 40, fig. 512, 1880; Dunker, Index Molluscorum, Maris Japonici, p. 5, 1880; Poirier, Rerision des Murex du Muséum, Nouvelles Archives du Muséum, D'Histoire Naturelle, Deuxièmo Série, Tome V, p. 91, No. 161, 1882 ; Watson, Report on the Gasteropoda, Toyage of H.M.S. "Challenger," Zoologỳ, Vol. XV, p. 155, 1886.

Murex (Alipurpura) acanthopterus, Bayle, 18s4, in Fischer, Manuel de Conchyliologie, Fascicule VII, p. 641, 1884.

Inebitat.-Watsons's Bay, Middle ITarbour, Bottle and Glass Rocks, Port Jackson; Port Stephens, New South Wales, under stones low water spring tides. (J. Brazier.) Near Cape York, Queensland, 6 fathoms, ('oral mud. (H.M.S. "Challenger.") Broken Bay. (A.MI.) Japan? (Dr. G. Dunker.) 


\section{II}

MLuex trigonutaris, Nowerby (not of Lamarek), Conchological Illustrations, species 56 , fig. 107,1810 .

Mrurex pellucidus, Reere, Proc. Zool. Soc., London, p. 87, 1815 ; Recre, Conch. Icon., Vol. III, pl. 1t, fig. 5\$, 18t5 ; Kuster, Conch. Cab., 2nd cdition, p. 96, p]. 33, fig. 7 .

Murex (Pleronolus) pellucills, Brizier, "Chevert" Expedition Shells, Proc. Limn. Soc, New South Wales, Vol. I, p. 171, No. 9, 1875, 1877; Kobelt, Jahrb. d. deutsch. malali. Gescllseh, Tol. IT, p. 160, not 130, 1877; 1'Tron, Manual of Conchology, Tol. II, p. 87, pl. 41, fig. 530, 1850 ; Poirier, Revision des Murex du Musèum, Nouvelles Archives du Muséum D’Histoire Naturelle, Deuxième Série, Tome V, p. 91, No. 160, 1852.

IIabitat.-Darnley Islank, Torres Straits, 30 fathoms, white, sandy inud bottom, brought up in the tangles. (J. Brazier, "Cherert" Expedition.)

\section{Murex osseus, Reeve.}

Minrex osseus, Reeve, Proc. Ziool. Soc., London, p. S7, 1S45; Conch. Icon., Tol. III, p]. 14, fig. 60, 1815 ; Kustel, Conch. Cab., 2nd edition, p 92, pl. 32, fig. 8. Sowerby, Thes. Conch., Vol. IV, p. 21, pl. 10, Murex, fig. 91.

MLuiex (Pteronotus) osseus. Kobelt, Jahrb. d. deutsch. malak. Gesellsch., Tol. IV, p. 160, No. 131, 1S77; Tryon, Manual of Concholngy, Tol. II, p. 87, pl. 41, fig. 525, 1550: Poirier, Revision des Murex du Muséum Nourelles Archives du MIsśum D’Hictoire Naturelle, Denxième, Nérie V, p. 93, 1852.

Habitat.-Nicol Bas, north-rest coast of Australia. (A.M.) (II. II. IIargrares.)

\section{Murex Angasi, Crosse.}

Typhis Angasi, Crosse, Joumal de C'onch., Tol. XT, p. S(;, pl. 1, fig. 2. 1S6:3. Murex (Plcronotus) angasi, Angas, Proc. Zool. Soc., London, p. 186, No. 5, 1867.

Murex zonatus, Tenison-1Toods, Proc. Roy. Soc., Tasmania, p. 132, 1s76.

Typhis Angasi, Crosice, Kobelt in Jahrb. d. dentsch. malak. Gesellsch., Tol. IV, p. 28s, No. 2, 1877.

Murex cos, II utton, Journal de Conch., Vol. TVIII. Brd series, p. 12, 157s Murex Angasi, Sowerby, Thes. Conch., Tol. 1T, p. 26, pl. 21, Murex, fig $189,190,1879$.

Murex (Pteronotus) angasi, Tryon; Manual of Conchology, Tol II, p. SS, pl. 40, fig. 522, 1580; Poirier, Revision des MLurex du Muséum, 
Nourelles Archives du Muséum D'Histoire Naturelle, Deuxìme Série, Tome V., p. 95, 1882; Bednall, Transactions and Proceedings and Report of the Royal Society of South Australia, Vol. VIII, p. Cit, 1585 1886; Brazier, Proc. Linn. Soc. of New South Wales, Vol. IT (2nd series), p. 993, No. 2, 1887.

Habitat.-Port Jackson, Broken Bay. (A.M.) Port Stephens, New South Wales; Tasmania; Victoria; South Australia; New Zealand; Norfolk Island.

\section{Murex bipunctatus, Sowerby.}

IIurex bipunctatus, Sowerby, Thes. Conch., Vol. IV, p. 22, pl. 21, Minex, fig. 188.

Murex (Pteronotus) bipunctatus, Tryon. Manual of Conchology, Vol. II, p. 85; pl. 4.0, fig. 507, 1980; Poirier, Revision des Murex du Muséum, Noturelles Archives du Muséum D'Histoire Naturelle, Deuxiime Série,

Tome $\mathrm{T}$, p. $95,1882$.

Habitat.-Australia. (G. B. Sowerby.)

\section{Murex cordismei, Wat:on.}

IFurex (Pteronotus) contismei, Watson, Prelim. Report, Part KT, Journal Linnean Society, London, Vol. XVI, p. 601. Watson, Report on the Gasteropoda, Voyage of H.MI S. "Challenger," Zoology, Vol. IV, p. 156, pl. 10, fig. 5, 1886.

ILabitat.-Off East Monceur Island, Bass Strait, 35 fathome, sand and shells. (H.M.S. "Challenger.")

\section{HOAIALOCANTHA, Morch, 1852. \\ INurex varicosus, Sowerby.}

IIUrex varicosus, Sowerby, Proc. Zool. Soc., London, p. 145, 1810; Sowerby. Conchological Illustrations, species 76, fig. 49, 1841; Kiener, Iconographie Coquilles Vivantes, p. 91, pl. 28, fig. 2, 1842 ; Reeve, Conch. Icon., Vol. III, pl. 12, fig. 49, 1845; Sowerby, Thes. Conch., Vol. IV, p. 31, Murex pl. 15, fig. 139, 1879.

Murex (IIomalocantha) varicosus, H. and A. Adams, Genera of Recent Mollusea, Vol. I, part III, p. 74, 1853, 1858; Kobelt, Catalog der Gattung Murex in Jabrb. d. deutsch. malak. Gesellsch., Vol. IV, p. 159, No. 121, 1877; Tryon, Manual of Conchology, Vol. II, p. 99, pl. 25, fig. 224, 1850; Poirier, Revision des Murex du Muséum, Nourelles Archives du Muséum D'Histoire Naturelle, Deuxième Série, Tome V, p. 89, No. 154, 1882.

Hubitut.-Nicol Bay, North-west A ustralial. (IV. II. Hargraves, A.MI.) 
OCINEBRA, Leach, 1817 ; TRTTONLLIA, Fleming, Ists.

Murex tetragonus, Broderip.

Inrex tetragonus, Broderip, Proc. Zool. Soe., London, p. 17 t, 1432 ; Suwerby, Conchological Illustrations, species 111, figs. 25, 36, 1811 ; Kiener, Iconngraphie, Coquilles, p. 87, pl. 5, fig. 2, 18t2; Kuster, Conch. Cab., 2nd edition, p. 65. pl. 24, fig. 3.

Inrex (Ocinebra) telugonats, II. and A. Adam, Genera of Recent Moliusca, Part IV, Vol. I, p. 75, 15.53 ; Śchmeltz, jr., Musem (rodeffror, C'atilogr T, p. 1:31, \o. 3,900, 1.57.t ; Kobelt, Jahrb. d. dentsch. malak. Cievellseh. Tol. IT, p. 2.2, To. 261, 1577; Brazier, "Chevert" Frpedition, Shells,

Pro. Linu. Soc., New South Wales, Vol. I, p. 171, No. 10, 1877, 1878; IIurex tetragonus, Sowerby, Thes. Conch., Vol. IV: p. 40, No. 1Ss, Murex, pl. 33, fig. 223, 1879; Liénard, Catalogue de la Faune Malacologique, de l' ile Maurice, p. 2, No. 15, 1877.

BLvex (Ocinebra) tetragonus, E. v. Martens in Möbius Beitrïge zur Mecresfauma der Insel Mauritius und der Sejchellen, 1. 232, 15s0: Tryon. Manual of Conchology, Vol. II, p. 121, pl. 36, fig. 424, 1.580.

IIurex (Ptcronotus) tetragonus, Tapparone-Canefri, La Faunc IJalacologiguc de l' ile Maurice, Mémoires de la Société Malacologirpue de Belgique. Tome XV (Deuxième Série, Tome V), p. 19, 1850.

Murex (Muricider) tetrayonus, Poirier, Revision des Murex du M[uséum, Nouvelles Arehives du Muséum D'ITistoire Naturelle, Deuxiène Súrie, Tome V, p. 102, No. 199, 1882.

Habitat.-Bet. Island, Torres Straits, 11 fathoms, sandy bottom. $(\cdot \mathrm{T}$ Brazier.)

\section{Murex mundus, Reeve.}

Murex exignus, Reeve, Proc. Zool. Soe., 1). ?, 1545. Recve (non Broulerip, Kiener nee Garrett), Conch. Icon., Tol. IIJ, pl. 32, species 1lit, 1S1J.

Wurex munilus, Reeve, Conch. Teon. (Errata in Index species 1606).

Mrurex (Muricidea) mundus, II. and A. Adams, Genera of Recent MIollusear, Vol. I, Part III, p. 75, 1853, 1858; Brazier, "Chevort" Expedition, Shells, Proc. Linn. Soc., New South Wales, Tol. I, p. 172, No. 12, $1877,1878$.

ALurex (Ocinebia) mundus, Kóobelt, Jahrb. d. deutsch. malak. Gescollsch., Vol. IV, p. 249, No. 237, 187'; Tryon, Manual of Conchology, Tol. II, pl. 36, fig. 427, 1 Sso. 
MIurex mundus, Sowerby, Thes. Conch, Vol. IV, p. 39, No. 182, Murer, pl. 33, fig. 242, 1879.

Inabitat.-Darnley Island, Torres Straits, 20 fathoms, coral bottom. Brazier.)

This species is not described in the Proc. Zool. Society, as quoted by Mr. Reere in Conch. Icon.

\section{Murex Brazieri, Angas.}

Murex Brazieri, Angas, Proc. Zool. Soc., London, p. 171, pl. 26, fig. 3, 1877; A further List of Additional Species of Marine Mollusca to be included in the fauna of Port Jackson and the adjacent coasts of New South Wales, Proc. Zool. Soc., London, p. 179, No. 12, 1877; Somerby, Thes. Conch., Vol. IV, p. 42, species 200, Murex, pl. 33, fig. 226 enlarged, 1879 ; Whitelegge, List of the Marine and Fresh Water Invertebrate Fiuna of Port Jackson and neigbbourhood, Journal and Proceedings of: the Royal Society, New Sonth Wales, Vol. XXIII, p. 246, No. 209, 1899 ; Brazier, Journal of Conchology, Vol. VI, p. 66, 1ฯ\$9.

ILurer (Ocinebra) Brazicri, Tryon, Manual of Conchologg, Vol. II, p. 132, pl. 30, fig. 289, 1880.

Alurex (Anricilea) Brazieri, Poirier, Rérision des MIurex du Muséum, Nourelles Archires du Muséum d'Histoire Naturelle, Deusième Série, Tome V, p. 104, No. 208, 1852.

Habitat.-Dredged outside Port Jackson Heads in 20 fathoms. (J Brazier, 1873.) Middle Harbour, in shell sand. (Whitelegge.) South Australia. (W. T. Beduall.) (A.M.)

This species was sent to me by Mr. Bednall as the young of Mrurex pumilus, A. Adams, the latter species not being found in South Australia, but in Chiua and Darros Island, Amirantes, in 22 fathoms.

\section{Murex planiliratus, Reeve.}

Murex planiliratus, Reere, Conch. Icon., pl. 31, fig. 149, 1845.

Aurex (Ocinebra) planiliratus, H. and A. Adams, Genera of Recent Mollusca, Vol. I, Part III, p. 7., 1853, 1858; Kobelt, Catalog der Gattung Murex in Jahrb. d. deutsch. malak. Gesellsch., Vol. IV, p. 248, No. 226, 1877.

Murre planiliratus (Hinds?), Sowerby, Thes. Coneh., Vol. IV, p. 19, Murex, pl. 24, fig. 264, 1879.

Coralliophila costularis, Tryon (non Lamarek), Mimual of Conchology, Vol. II, p. 208, pl. 65, fig. 366, 1880. 
Murex (Ocinebra) plantiratus, Poirier, Revision des Murex du MIúium,

Nourelles Archives du Musćum d'Historie Naturelle, Deuxième Śrie, Tome V, p. 126, No. 285, 1882.

Habitat.-Swan Rirer, Western Australia. (Recre.) Tear Fremantle. (Dr. J. C. Cox, 1892.)

Murex serotinus, A. Adams.

Murcx serotinus, A. Adams, Proc. Zool. Soc., London, p. 26S, 1 S51.

Murex (Ocinebra) serotinus, H. and A. Adams, Genera of Recent Mollusea, Vol. I, Part III, p. 75 ; 1853, 1858.

Murex scrotinus, Angas, Molluscan Fauma of South Australia, Proc. Zuvl. Soc., London, p. 157, No.4, 1865.

MInex serotinus = Blainvillei? Sowerby in Thes. Conch., Tol. IV, index p. 51, 1879.

Murex serotinus $=$ Murex eristatus Tar. Blainvillei, Tryon in Manual of Conchology, Vol. II, p. $25 \pm$ index, 1880.

Murex serotimus, Bednall, Transactions and Proceedings and Report of the Rojal Society of South Australia, Vol. VIII, p. 66, 1SS1-5, issued May, 1886.

Habitat.-Aldinga Bay, South Australia. (G. F. Angas.)

\section{VITULARIA, Swainson, 1840.}

Vitularia miliaris, Gmelin.

Murcx Pupura scabra, Chemuitz, Conch. Cab., Tol. X, pp. 231, 219, pl. 161, fig. $1,532,1,535 ; 1788$.

Murex miliaris, Gmelin, Srst. Nat., Tol. I, Part VI, p. 3,536, No. 39, 1790; Dillwyn, Descriptive Catalogue of Recent Shells, Tol. II, p. 655, No. $10,1817$.

MLurex vitulinus, Lamarek, Tableau Encyclopédique Méthodique, pl. 419, fig. 1, a b; 7 a b, 1816; Lamarek, Anim. sans Vert., Vol. VII, Part I, p. 173, No. 53, 1822; 2nd edition Deshayes, Vol. IX, p. 595, No. 53, 1S13. Deshayes, in Eneyclopédie Méthouliyue, Histuire Naturelle de's Vers, Vol. III, p. 905, No. 24, 1832; Kiener, Coquilles Virantes, p. 123, pl. 17, fig. 2, 1512; Menke, Molluscorum Novie IIollandia', p. 2t, No. 11t, 1813; Kuster, Conch. Cal., 2nd edition, p. 1s, pl. 19, figs. 5, s. Murex Purpura, Reeve, Conch. Icon., Vol. III, pl. 25, fig. 102, 1815.

Titulario mitiaris, Kobelt. Catalog der Gattung Vitularia in Jilhrb. d. deutsch. malak. Gesellsch., Vol. IV, p. 252, No. 1, 1877. 
ILurex (Titularia) miliaris, Tryon, Manual of Conchology, Vol. II, p. 133, pl. 35, figs. 393, 387; 1880.

Habitat.-Nicol Bay North, West Australia; Osnaburg Point, Albany Pass, Cape York, North Queensland found under stones at low water. (J. Brazier, "Chevert" Expedition, 1875.) $)^{\ell}$ Torres Straits. (W. H. Hargraves, A.M.)

This species was inadrertently omitted by me from the Muricide of the "Cherert" Expedition Proceedings of the Limmean Society of New South Wales, Vol. I, pp. 169, $172 ; 1875,1877$.

\section{PSEUDOMUREX, Monterosato, 1872.}

Murex pholidotus, Watson.

IIurex (Ocincbra) pholidotus, Watson, Prelim. Report, part 15, Joumal Linnean Society, London, Tol. XVI, p. 602, 1883.

IIuvex (Pscudomutrex) pholidotus, Thatson, Report on the Gasteropoda Toyage of II.\I.S. "Challenger" Zoology, Tol. XV, p. 15s, pl. X, fig. 3, 1886 .

Habitat.-Flinders Passage, Cape York, Torres Straits, 7 fathoms. (H.M.S. "Challenger.")

Mirex polypleurus, Brazier, N. Sp.

BLurcx polyplcurus, Brazier, Proc. Linn. Soe, New South Wales, Second Series, Vol. VIIT, Part I, 1893.

Habitat.-MacDonnell, Fowler's Bay (Professor Tate). East side of St. Tincent's Gulf, South Australia (G. F. Augas). Port Lincolu (W. T. Bednell).

TYPHIS, Montfort, 1810.

Typhis arcuatus, Hinds.

Typhis arcuatus, Hinds, Proc. Zool. Soc., London, p. 19, 1\$43. Hindds, Toyage of II.M.S. "Sulphur," Mollusea, p. 10, pl. 3, fig. 1, 2 ; Sowerby, Thes. Conch., Vol. III, p. 320, No. 11, J'hes. pl. 281, fig. 3, 4, 5 ; Reeve, Conch. Icon., Tol. XIX, pl. 3, fig. 10, $187 \frac{1}{2}$; Tenison-Woods, Papers and Procecdings Royal Society, Tasmania, p. 132, 1S76; Kobelt, Catalog der Gattung Typhis in Jahrb. d. deutsch. malak. Gesellsch., Vol. IV, p. 2S?, No. 14, 1877 ; Brazier, Proc. Limn. Soc., New South WVales, Tol. IV, p. $428 ; 1879,80$.

Typhlis Japonicus, A. Adams, Proc. Zool. Soc., London, p. 37t, 1862. 
Typhis arcuatus, Tryon, Manual of Conchology, Vol. II, p. 136, pl. 30, fig. 293, 1880 ; Whitelegge, List of the Marine and Fresh Water Invertebrate Fauna of Port Jackson and Neighbourhood, Journal and Proceedings of the Royal Society of New South Wales, Vol. XXIII, p. 246, No. 211, 1889.

Habitat.-Cape of Good Hope, dredged on the L'Agulhas Bank, in from 40 to 44 fathoms (Mr. R. B. Hinds). Outer North Head of Port Jackson, near the rocks, 5 fathoms sand and shells, "Sow and Pig's Reef," 3 fathoms (J. Brazier, 1865). Long Bay, Bruny Island, Tasmania, 10 fathoms, sand bottom (Rev. H. D. Atkinson). Japan (A. Adams).

\section{Typhis Cleryi, Sowerby.}

Typhis Cleryi, Sowerby (non Petit), Thes. Conch, Vol. III, p. 320, Thes. pl. 284, fig. 14 .

Typhis Clerii.-Sowerby in Reeve, Conch. Icon., Vol. XIX, pl. 3, fig 11, 1874. Typhis Cleryi.-Brazier (non Petit), Proc. Linn. Soc., New South Wales, Vol. I, p. 9, 1975, 1877 ; Tryon (non Petit), Manual of Conchology, Vol. II, p. 137, pl. 30, fig. 302, 1880 ; Hutton (non Petit), Manual of the New Zealand Mollusea, p. 47, 1880.

Typhis philippensis, Watson, Prelim. Report, Part XV, Journal Linnean Society, London, Vol. XVI, p. 605, 1883 ; Report on the Gasteropoda, Voyage of H.M.S. "Challenger," Zoology, Vol. XV, p. 162, pl. 10, fig. $4,1856$.

Typhis Cleryi, Sowerby, Whitelegge, List of the Marine and Fresh Water Invertebrate Fauna of Port Jackson and Neighbourhood, Journal and Proceedings of the Royal Society, New South Wales, Vol. XXIII, p. 246, No. 210, 1889.

Habitat.-Off Sydney Heads, 5 miles due east, 45 fathoms sandy bottom, June 3rd, 1874; Jervis Bay, 70 miles south of Sydney, 11 fathoms sand and gravel, October 24, 1874. (J. Brazier.) (A.M.) Station 161, April 1, 1874. Lat. $38^{\circ} 30^{\prime \prime}$ S., long. $144^{\circ} 36^{\prime} 30^{\prime \prime}$ E. Off the entrance to Port Phillip, Melbourno, 33 fathoms sand. (H.M.S. "Challenger.")

This species is not found in New Zealand as quoted by Mr. Sowerby in the Thesaurus Conchyliorum and the Conchologia Iconica on the authority of Mr. G. F. Angas, the specimen came from New South Wales.

Typhis Cleryi, Petit, 1840, is a synonym of Typhis Btcheri, Broderip, 1832.

Typhis phitippensis, Watson, cannot be separated from Typhis Cleryi, Sowerby. 
Typhis Yatesi, Crosse.

Typhis Yatesi, Crosse, Journal de Conch., Vol. XIII, p. 5t, pl. 2, fig. 3, 1865 ; Angas, Molluscan Fauna of South Australia, Proc. Zool. Soc., London, p. 158, No. 7, 1865; Sowerby, Thes. Conch., Vol. III, p. 319, Thes., pl. 281, figs. 20, 21; in Reeve, Conch. Ieon., Vol. XIX, pl. 3, fig. 14, $187 t$.

Typhis Yatesii, Kobelt, Catalog der Gattung Typhis in Jahrb. 1. deutsch. malak. Gesellsch., Vol. IV, p. 288, No. 3, 1877 ; Tryon, Manual of Conchology, Vol. II, p. 137, pl. 30, fig. 294, 1880.

Typhis Yatesi, Bednall, Transactions and Proceedings and Report of the Royal Society of South Australia, Vol. VIII, p. 66, 1884-5, issued May, 1886.

Habitat.-Holdfast Bay, Semaphore, St. Vineent's Gulf, South Australia. (Angas, Tate, Bednall, Forwood.) Stansbury, South Australia. (Professor Tate.) 



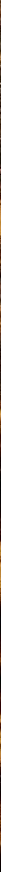

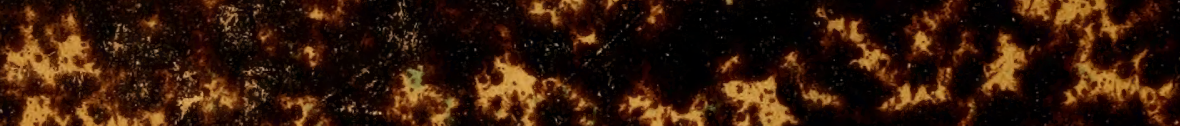

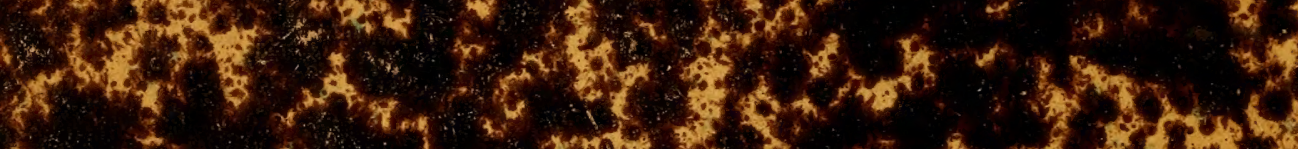

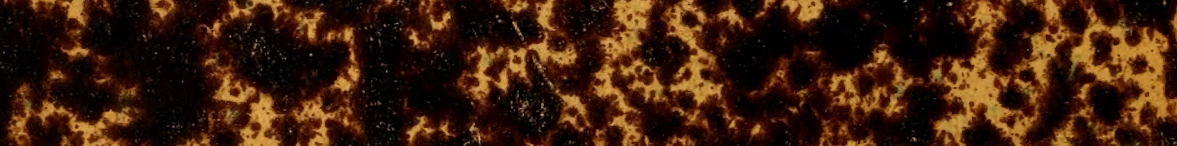

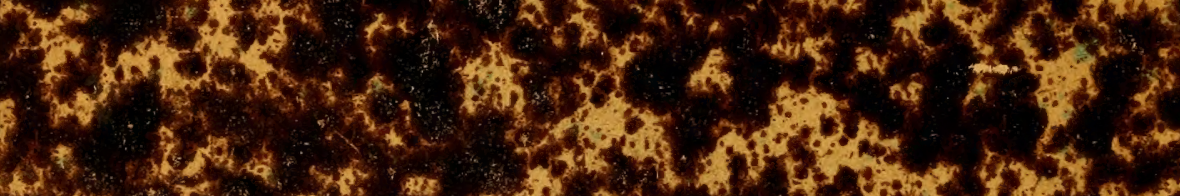

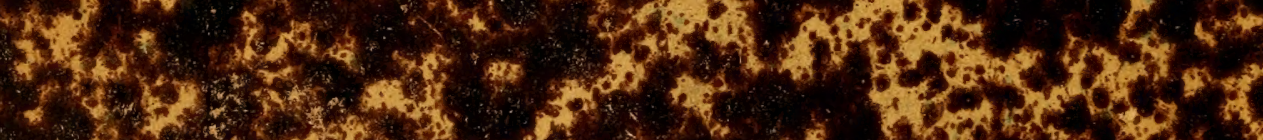

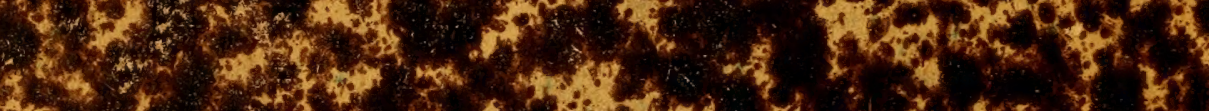

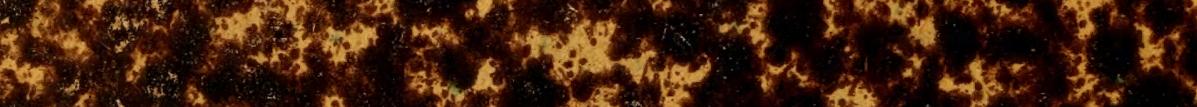

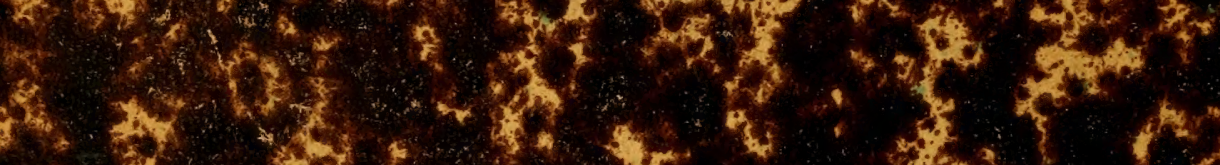

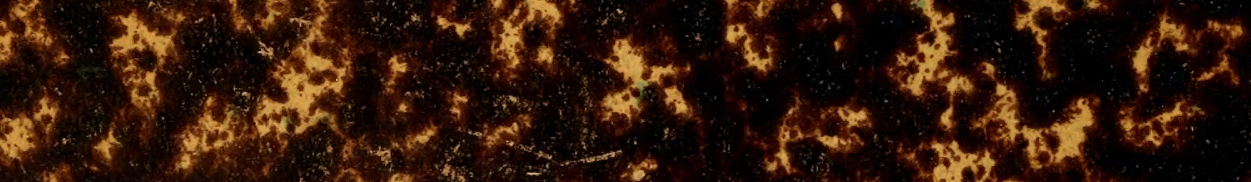

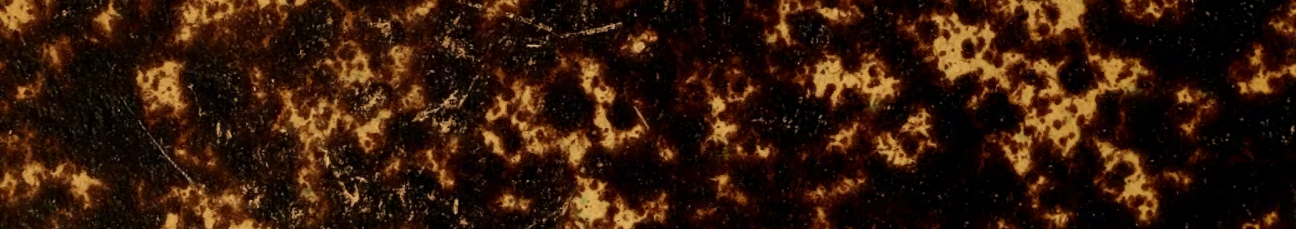

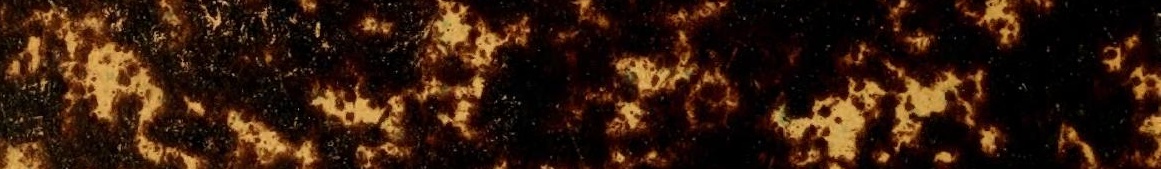

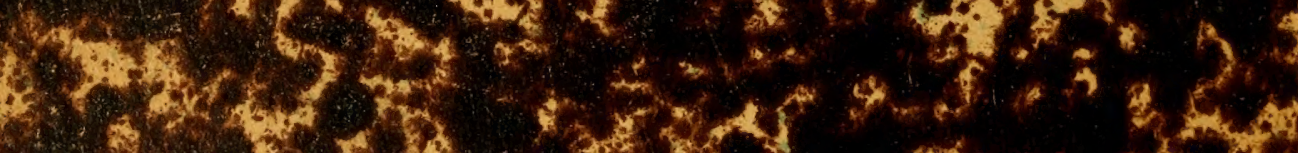

